\title{
Supporting Information for \\ Homologation Method for Preparation of Substituted Pentacenes and Naphthacenes
}

Tamotsu Takahashi, ${ }^{\text {a* }}$ Shi Li, ${ }^{a}$ Wenying Huang, ${ }^{a}$ Fanzhi Kong, ${ }^{a}$ Kiyohiko Nakajima, ${ }^{b}$ Baojian Shen, Takahiro Ohe, ${ }^{\mathrm{d}}$ and Ken-ichiro Kanno ${ }^{\mathrm{a}}$

${ }^{a}$ Catalysis Research Center and Graduate School of Pharmaceutical Sciences, Hokkaido University, and SORST, Japan Science and Technology Corporation (JST), Kita-ku Sapporo 001-0021, Japan

${ }^{\mathrm{b}}$ Department of Chemistry, Aichi University of Education, Igaya, Kariya, Aichi, 448-8542, Japan

${ }^{c}$ Petroleum Univ.-Hokkaido Univ. Joint Lab, Faculty of Chemical Science and Engineering China University of Petroleum-Beijing, Changping, Beijing, 102249, China.

${ }^{\mathrm{d}}$ Materials Laboratories, Sony Corporation, Okata, Atsugi, Kanagawa 243-0021 Japan

Tamotsu@cat.hokudai.ac.jp

\section{CONTENTS}

General

S2

Preparations and Spectral Data of Compounds

Type I pentacenes 9a and 9c (3c, 4c, 5a, 5c, 6a, 6c, 7a, 7c, 8a, 8c, 9a, 9c) $\quad$ S2 - S4

Type II pentacene 9d (4d, 5d, 6d, 7d, 8d, 9d) S5 - S6

$\begin{array}{ll}\text { Other intermediates }(\mathbf{5 e}, \mathbf{8 e}, \mathbf{1 1} \mathbf{a}, \mathbf{1 1 b}, \mathbf{1 4}) & \text { S6 - S7 }\end{array}$

NMR Charts

Bis(propargyl)benzenes 3b, 3c, 3e

$\mathrm{S} 8-\mathrm{S} 10$

Dihydroanthracenes $4 \mathbf{b}, \mathbf{4 c}, \mathbf{4 d}, \mathbf{4 e}$

$\mathrm{S} 11-\mathrm{S} 14$

Anthracenes 5a, 5b, 5c, 5d, 5e

$\mathrm{S} 15-\mathrm{S} 19$

Bis(bromomethyl)anthracenes 6a, 6b, 6c, 6d, 6e

$\mathrm{S} 20-\mathrm{S} 24$

Bis(propargyl)anthracenes 7a, 7b, 7c, 7d, 7e

$\mathrm{S} 25-\mathrm{S} 29$

Dihydropentacenes 8a, 8b, 8c, 8d, 8e

$\mathrm{S} 30-\mathrm{S} 34$

Pentacenes 9a, 9b, 9c, 9d, 9e, 9f, 9g, 9h, 9i

$\mathrm{S} 35-\mathrm{S} 43$

Dihydroanthracenes 4g, 4f, 4h, 10

$\mathrm{S} 44-\mathrm{S} 47$

Tetrahydropentacenes 11a, 11b, 12a, 12b, 12c, 12d, 12e

S48 - S54

Bis(propargyl)naphthalene 14

S55

Dihydronaphthacenes 15a, 15b

S56 - S57

Naphthacenes 16a, 16b

S58 - S59

UV-vis Absorption Spectra of Selected Pentacenes

Pentacenes 9a, 9b, 9c, 9d, and 9g (in solution)

$\mathrm{S} 60-\mathrm{S} 62$

Pentacenes 9f, 9h, and 9i (in solid state)

S62 


\section{General.}

Manipulations were carried out under a nitrogen atmosphere using standard Schlenk techniques. Tetrahydrofuran (THF) was dried over sodium and benzophenone under a nitrogen atmosphere. All of the reagents were commercially available and used as received until otherwise mentioned. Compounds 1, 2a, 3a, and 4a were prepared according to the literature. ${ }^{1}$ GC yields were determined using suitable hydrocarbons as internal standards.

[1] Takahashi, T.; Kitamura, M.; Shen, B.; Nakajima, K. J. Am. Chem. Soc. 2000, 122, 12876.

\section{Preparation of 1,2-bis(2-heptynyl)-3,4,5,6-tetraethylbenzene (3c) from 2a}

The compound 3c was prepared in 72\% yield from dibromide $\mathbf{2 a}$ and 1-hexyne by the same way as described for $\mathbf{3 b}$.

3c: a pale-yellow oil; ${ }^{1} \mathrm{H}$ NMR $\left(\mathrm{CDCl}_{3}, \delta\right) 0.89(\mathrm{t}, 6 \mathrm{H}, J=7 \mathrm{~Hz}), 1.19(\mathrm{t}, 6 \mathrm{H}, J=7 \mathrm{~Hz}), 1.23(\mathrm{t}, 6$ $\mathrm{H}, J=7 \mathrm{~Hz}), 1.30-1.52(\mathrm{~m}, 8 \mathrm{H}), 2.12(\mathrm{tt}, 4 \mathrm{H}, J=7,2 \mathrm{~Hz}), 2.67$ (q, $J=7 \mathrm{~Hz}, 4 \mathrm{H}), 2.75(\mathrm{q}, J=7$ $\mathrm{Hz}, 4 \mathrm{H}), 3.62(\mathrm{t}, 4 \mathrm{H}, J=2 \mathrm{~Hz}) ;{ }^{13} \mathrm{C} \mathrm{NMR}\left(\mathrm{CDCl}_{3}, \delta\right)$ 13.6, 15.4, 15.7, 18.7, 19.2, 22.0, 22.3, 22.7, 31.1, 78.3, 80.6, 133.0, 138.3, 138.9; HRMS calcd for $\mathrm{C}_{28} \mathrm{H}_{42}$ : 378.3286, found: 378.3289 .

\section{Preparation}

\section{1,4-dibutyl-2,3-bis(methoxycarbonyl)-5,6,7,8-tetraethyl-9,10-dihydroanthracene (4c) from 3c}

The compound $\mathbf{4 c}$ was prepared in $58 \%$ yield from diyne $\mathbf{3 c}$ by the same way as described for $\mathbf{4 b}$. 4c: colorless crystals, $\mathrm{mp}=75.9-78.4{ }^{\circ} \mathrm{C} ;{ }^{1} \mathrm{H} \mathrm{NMR}\left(\mathrm{CDCl}_{3}, \delta\right) 0.99(\mathrm{t}, 6 \mathrm{H}, J=7 \mathrm{~Hz}), 1.19$ (t, $6 \mathrm{H}, J$ $=7 \mathrm{~Hz}), 1.23(\mathrm{t}, 6 \mathrm{H}, J=7 \mathrm{~Hz}), 1.47(\mathrm{tq}, 4 \mathrm{H}, J=7,7 \mathrm{~Hz}), 1.54-1.67$ (m, $4 \mathrm{H}), 2.69$ (q, $J=8 \mathrm{~Hz}, 4$ $\mathrm{H}), 2.75-2.88(\mathrm{~m}, 8 \mathrm{H}), 3.84(\mathrm{~s}, 6 \mathrm{H}), 3.92(\mathrm{~s}, 4 \mathrm{H}) ;{ }^{13} \mathrm{C} \mathrm{NMR}\left(\mathrm{CDCl}_{3}, \delta\right)$ 13.8, 15.3, 15.9, 22.30, 22.31, 23.1, 29.5, 30.2, 33.1, 52.2, 130.0, 132.3, 135.3, 136.4, 137.9, 139.2, 169.6; HRMS Calcd for $\mathrm{C}_{34} \mathrm{H}_{48} \mathrm{O}_{4}: 520.3552$; Found: 520.3556.

\section{Preparation of 1,4,5,6,7,8-hexaethyl-2,3-bis(methoxycarbonyl)anthracene (5a) from 4a}

The compound $\mathbf{5 a}$ was prepared by the similar way as described for $\mathbf{5 b}$ in $62 \%$ isolated yield from dihydroanthracene $\mathbf{4 a}$.

5a: ${ }^{1} \mathrm{H}$ NMR $\left(\mathrm{CDCl}_{3}, \delta\right) 1.30(\mathrm{t}, J=7.5 \mathrm{~Hz}, 6 \mathrm{H}), 1.42(\mathrm{t}, J=7.8 \mathrm{~Hz}, 6 \mathrm{H}), 1.48(\mathrm{t}, J=7.5 \mathrm{~Hz}, 6 \mathrm{H})$, $2.91(\mathrm{q}, J=7.5 \mathrm{~Hz}, 4 \mathrm{H}), 3.23-3.34(\mathrm{~m}, 8 \mathrm{H}), 3.94(\mathrm{~s}, 6 \mathrm{H}), 8.90(\mathrm{~s}, 2 \mathrm{H}) ;{ }^{13} \mathrm{C} \mathrm{NMR}\left(\mathrm{CDCl}_{3}, \delta\right) 15.5$, $15.7,15.8,22.0,23.0,23.8,52.3,121.3,126.2,128.6,130.3,135.1,138.8$ (2C), 169.8 .

\section{Preparation of 1,4-dibutyl-2,3-bis(methoxycarbonyl)-5,6,7,8-tetraethylanthracene (5c) from $4 \mathrm{c}$}

The compound $\mathbf{5 c}$ was prepared in $90 \%$ yield from dihydroanthracene $\mathbf{4 c}$ by the same way as described for $\mathbf{5 b}$.

5c: pale-yellow crystals; $\mathrm{mp}=90.0-91.0{ }^{\circ} \mathrm{C} ;{ }^{1} \mathrm{H} \mathrm{NMR}\left(\mathrm{CDCl}_{3}, \delta\right) 0.93(\mathrm{t}, 6 \mathrm{H}, J=7 \mathrm{~Hz}), 1.20$ (t, 6 $\mathrm{H}, J=7 \mathrm{~Hz}), 1.32$ (t, $6 \mathrm{H}, J=7 \mathrm{~Hz}), 1.48$ (td, $4 \mathrm{H}, J=7,7 \mathrm{~Hz}), 1.68-1.73$ (m, $4 \mathrm{H}), 2.81$ (q, $4 \mathrm{H}, J$ 
$=7 \mathrm{~Hz}), 3.09-3.24(\mathrm{~m}, 8 \mathrm{H}), 3.83(\mathrm{~s}, 6 \mathrm{H}), 8.78(\mathrm{~s}, 2 \mathrm{H}) ;{ }^{13} \mathrm{C} \mathrm{NMR}\left(\mathrm{CDCl}_{3}, \delta\right) 13.9,15.4,15.7,22.0$, 23.0, 23.3, 30.2, 35.5, 52.2, 121.4, 126.5, 128.9, 130.3, 135.1, 137.6, 138.8, 169.8; Anal. Calcd for $\mathrm{C}_{34} \mathrm{H}_{46} \mathrm{O}_{4}$ : C, 78.72; H, 8.94; Found: C, 78.53; H, 9.00.

\section{Preparation of 1,4,5,6,7,8-hexaethyl-2,3-bis(bromomethyl)anthracene (6a) from 5a}

The compound $\mathbf{6 a}$ was prepared in $86 \%$ yield from diester $\mathbf{5 a}$ by the same way as described for $\mathbf{6 b}$. 6a: ${ }^{1} \mathrm{H}$ NMR $\left(\mathrm{CDCl}_{3}, \delta\right) 1.30(\mathrm{t}, J=7.5 \mathrm{~Hz}, 6 \mathrm{H}), 1.41(\mathrm{t}, J=7.5 \mathrm{~Hz}, 6 \mathrm{H}), 1.49(\mathrm{t}, J=7.5 \mathrm{~Hz}, 6 \mathrm{H})$, 2.90 (q, $J=7.5 \mathrm{~Hz}, 4 \mathrm{H}), 3.26$ (q, $J=7.5 \mathrm{~Hz}, 4 \mathrm{H}), 3.37$ (q, $J=7.8 \mathrm{~Hz}, 4 \mathrm{H}), 5.00$ (s, $6 \mathrm{H}), 8.80$ (s, $2 \mathrm{H}) ;{ }^{13} \mathrm{C} \mathrm{NMR}\left(\mathrm{CDCl}_{3}, \delta\right) 15.2,15.5,15.8,21.9,22.2,23.0,29.7,120.6,128.9$ (2C), 130.0, 135.0, $138.3,140.0$.

\section{Preparation of 1,4-dibutyl-2,3-bis(bromomethyl)-5,6,7,8-tetraethylanthracene (6c) from 5c}

The compound $\mathbf{6 c}$ was prepared in $83 \%$ yield from anthracene $\mathbf{5 c}$ by the same way as described for 6b.

6c: Yellow powder; mp $=185.8-186.8{ }^{\circ} \mathrm{C} ;{ }^{1} \mathrm{H} \mathrm{NMR}\left(\mathrm{CDCl}_{3}, \delta\right) 1.09(\mathrm{t}, 6 \mathrm{H}, J=7 \mathrm{~Hz}), 1.30(\mathrm{t}, 6 \mathrm{H}$, $J=7 \mathrm{~Hz}), 1.41$ (t, $6 \mathrm{H}, J=7 \mathrm{~Hz}), 1.68(\mathrm{tq}, 4 \mathrm{H}, J=7,7 \mathrm{~Hz}), 1.75-1.93$ (m, $4 \mathrm{H}), 2.89$ (q, $4 \mathrm{H}, J=8$ $\mathrm{Hz}), 3.18-3.38$ (m, $8 \mathrm{H}), 4.99$ (s, $4 \mathrm{H}), 8.78(\mathrm{~s}, 2 \mathrm{H}) ;{ }^{13} \mathrm{C} \mathrm{NMR}\left(\mathrm{CDCl}_{3}, \delta\right)$ 14.0, 15.5, 15.8, 22.0, 23.0, 23.6, 29.1, 30.0, 33.2, 120.6, 129.1, 129.2, 129.9, 135.0, 138.3, 138.9; Anal. Calcd for $\mathrm{C}_{32} \mathrm{H}_{44} \mathrm{Br}_{2}$ : C, 65.31; H, 7.54; Found: C, 65.48; H, 7.60.

\section{Preparation of 1,4,5,6,7,8-hexaethyl-2,3-di-2-pentynylanthracene (7a) from 6 a}

The compound $7 \mathbf{a}$ was prepared in $78 \%$ yield from dibromide $\mathbf{6 a}$ and 1-butyne by the same way as described for $\mathbf{7 b}$.

7a: ${ }^{1} \mathrm{H} \mathrm{NMR}\left(\mathrm{CDCl}_{3}, \delta\right) 1.10(\mathrm{t}, J=7.2 \mathrm{~Hz}, 6 \mathrm{H}), 1.30(\mathrm{t}, J=7.5 \mathrm{~Hz}, 6 \mathrm{H}), 1.41(\mathrm{t}, J=7.5 \mathrm{~Hz}, 6 \mathrm{H})$, 1.44 (t, $J=7.5 \mathrm{~Hz}, 6 \mathrm{H}), 2.16$ (q, $J=7.2 \mathrm{~Hz}, 4 \mathrm{H}), 2.89$ (q, $J=7.5 \mathrm{~Hz}, 4 \mathrm{H}), 3.26$ (q, $J=7.5 \mathrm{~Hz}, 4$ H), 3.34 (q, $J=7.5 \mathrm{~Hz}, 4 \mathrm{H}), 3.85$ (s, $4 \mathrm{H}), 8.77$ (s, $2 \mathrm{H})$.

\section{Preparation of 1,4-dibutyl-2,3-bis(2-heptynyl)-5,6,7,8-tetraethylanthracene (7c) from 6c}

The compound $7 \mathbf{c}$ was prepared in $76 \%$ yield from dibromide $\mathbf{6 c}$ and 1 -hexyne by the same way as described for $\mathbf{7 b}$.

7c: Yellow powder; $\mathrm{mp}=153.5-155.0{ }^{\circ} \mathrm{C} ;{ }^{1} \mathrm{H} \mathrm{NMR}\left(\mathrm{CDCl}_{3}, \delta\right) 0.87(\mathrm{t}, 6 \mathrm{H}, J=7 \mathrm{~Hz}), 1.07$ (t, $6 \mathrm{H}$, $J=7 \mathrm{~Hz}), 1.18-1.50(\mathrm{~m}, 20 \mathrm{H}), 1.65(\mathrm{td}, 4 \mathrm{H}, J=7,7 \mathrm{~Hz}), 1.73-1.86(\mathrm{~m}, 4 \mathrm{H}), 2.06-2.17$ (m, $4 \mathrm{H})$, 2.89 (q, $4 \mathrm{H}, J=7 \mathrm{~Hz}), 3.17-3.32(\mathrm{~m}, 8 \mathrm{H}), 3.85(\mathrm{~s}, 4 \mathrm{H}), 8.75(\mathrm{~s}, 2 \mathrm{H}) ;{ }^{13} \mathrm{C} \mathrm{NMR}\left(\mathrm{CDCl}_{3}, \delta\right)$ 13.6, 14.0, 15.4, 15.9, 18.6, 20.1, 21.9, 22.0, 23.0, 23.6, 29.3, 31.1, 32.9, 78.4, 81.1, 119.7, 129.1, 129.2, 131.1, 134.6, 134.8, 137.0; HRMS Calcd for $\mathrm{C}_{44} \mathrm{H}_{62}$ : 590.4851; Found: 590.4858 .

Preparation of Dimethyl 1,4,5,7,8,9,10,12-octaethyl-13,14-dihydropentacene-2,3-dicarboxylate (8a) from 7a 
The compound 8a was prepared from diyne 7a by the same way as described for $\mathbf{8 b}$. Column chromatography on silica gel $\left(\mathrm{Et}_{2} \mathrm{O} /\right.$ hexane, $\left.1 / 10\right)$ afforded the title compound $\mathbf{8 a}$ as colorless crystals in $40 \%$ yield.

8a: ${ }^{1} \mathrm{H}$ NMR $\left(\mathrm{CDCl}_{3}, \delta\right)$ 1.27-1.36 (m, $\left.12 \mathrm{H}\right), 1.41-1.48(\mathrm{~m}, 12 \mathrm{H}), 2.86-2.96(\mathrm{~m}, 8 \mathrm{H}), 3.24-3.32(\mathrm{~m}$, $4 \mathrm{H}), 3.39-3.47(\mathrm{~m}, 4 \mathrm{H}), 3.86(\mathrm{~s}, 6 \mathrm{H}), 4.18(\mathrm{~s}, 4 \mathrm{H}), 8.79(\mathrm{~s}, 2 \mathrm{H}) ;{ }^{13} \mathrm{C} \mathrm{NMR}\left(\mathrm{CDCl}_{3}, \delta\right) 15.2,15.5$, 15.6, 15.9, 21.9, 22.0, 23.0, 24.0, 30.0, 52.3, 119.5, 128.6, 129.0, 130.1, 130.8, 133.1, 134.8, 136.4, 137.1, 139.7, 169.5; HRMS calcd for $\mathrm{C}_{42} \mathrm{H}_{52} \mathrm{O}_{4}: 620.3866$, found: 620.3869 .

\section{Preparation of 1,4,6,13-tetrabutyl-2,3-bis(methoxycarbonyl)-}

\section{8,9,10,11-tetraethyl-5,14-dihydropentacene (8c) from 7c}

The compound $\mathbf{8 c}$ was prepared in $54 \%$ yield from diyne $7 \mathbf{c}$ by the same way as described for $\mathbf{8 b}$.

8c: pale-yellow powder; $\mathrm{mp}=126.4-128.5{ }^{\circ} \mathrm{C} ;{ }^{1} \mathrm{H} \mathrm{NMR}\left(\mathrm{CDCl}_{3}, \delta\right) 1.03(\mathrm{t}, 6 \mathrm{H}, J=7 \mathrm{~Hz}), 1.10$ (t, $6 \mathrm{H}, J=7 \mathrm{~Hz}), 1.29$ (t, $6 \mathrm{H}, J=7 \mathrm{~Hz}), 1.43$ (t, $6 \mathrm{H}, J=7 \mathrm{~Hz}), 1.48-1.58$ (m, $4 \mathrm{H}), 1.58-1.73$ (m, 8 H), 1.73-1.88 (m, $4 \mathrm{H}), 2.81-2.97(\mathrm{~m}, 8 \mathrm{H}), 3.27$ (q, $4 \mathrm{H}, J=7 \mathrm{~Hz}), 3.32-3.43(\mathrm{~m}, 4 \mathrm{H}), 3.85(\mathrm{~s}, 6 \mathrm{H})$, $4.15(\mathrm{~s}, 4 \mathrm{H}), 8.78(\mathrm{~s}, 2 \mathrm{H}) ;{ }^{13} \mathrm{C} \mathrm{NMR}\left(\mathrm{CDCl}_{3}, \delta\right) 13.9,14.0,15.4,15.9,22.0,23.0,23.2,23.5,28.6$, 30.3, 30.5, 33.1, 33.3, 52.2, 119.5, 128.85, 128.91, 130.2, 131.1, 131.8, 134.7, 135.2, 137.1, 139.7, 169.5; Anal. Calcd for $\mathrm{C}_{50} \mathrm{H}_{68} \mathrm{O}_{4}$ : C, 81.92; H, 9.35; Found: C, 81.88; H, 9.43.

\section{Preparation of dimethyl 1,4,6,8,9,10,11,13-octaethylpentacene-2,3-dicarboxylate (9a) from 8a}

The compound $9 \mathbf{a}$ was prepared from dihydropentacene $\mathbf{8 a}$ by the same way as described for $\mathbf{9 b}$. Column chromatography on silica gel (firstly with $\mathrm{Et}_{2} \mathrm{O} /$ hexane, 1/5; then with $\mathrm{CHCl}_{3}, 100 \%$ ) afforded $80 \mathrm{mg}(0.5 \mathrm{mmol}$ scale $)$ of the title compound 9a as a deep blue solid. Isolated yield $26 \%$.

9a: ${ }^{1} \mathrm{H}$ NMR $\left(\mathrm{CDCl}_{3}, \delta\right) 1.32(\mathrm{t}, J=7.4 \mathrm{~Hz}, 6 \mathrm{H}), 1.48-1.58(\mathrm{~m}, 12 \mathrm{H}), 1.66(\mathrm{t}, J=7.5 \mathrm{~Hz}, 6 \mathrm{H})$, 2.90 (q, $J=7.5 \mathrm{~Hz}, 4 \mathrm{H}), 3.33$ (q, $J=7.5 \mathrm{~Hz}, 8 \mathrm{H}), 3.95(\mathrm{~s}, 6 \mathrm{H}), 4.03$ (q, $J=7.5 \mathrm{~Hz}, 4 \mathrm{H}), 9.16$ (s, $2 \mathrm{H}), 9.26(\mathrm{~s}, 2 \mathrm{H}) ;{ }^{13} \mathrm{C} \mathrm{NMR}\left(\mathrm{CDCl}_{3}, \delta\right) 15.4,15.7,15.8,15.9,22.0,22.2,23.1,23.8,52.3,119.9$, 122.6, 126.1, 127.3, 127.5, 128.2, 129.8, 134.7, 135.2, 137.7, 139.4, 169.7; HRMS calcd for $\mathrm{C}_{42} \mathrm{H}_{50} \mathrm{O}_{4}: 618.3709$, found: 618.3717 .

\section{Preparation of 1,4,6,13-tetrabutyl-2,3-bis(methoxycarbonyl)-8,9,10,11-tetraethylpentacene} (9c) from 8c

The compound 9c was prepared in $42 \%$ yield from dihydropentacene $8 \mathbf{c}$ by the same way as described for $\mathbf{9 b}$.

9c: dark-blue crystals; $\mathrm{mp}=181.2-182.0{ }^{\circ} \mathrm{C} ;{ }^{1} \mathrm{H} \mathrm{NMR}\left(\mathrm{CDCl}_{3}, \delta\right) 1.07(\mathrm{t}, 6 \mathrm{H}, J=7 \mathrm{~Hz}), 1.13(\mathrm{t}, 6$ $\mathrm{H}, J=7 \mathrm{~Hz}), 1.33(\mathrm{t}, 6 \mathrm{H}, J=7 \mathrm{~Hz}), 1.51(\mathrm{t}, 6 \mathrm{H}, J=7 \mathrm{~Hz}), 1.57-1.83(\mathrm{~m}, 8 \mathrm{H}), 1.84-2.10(\mathrm{~m}, 8 \mathrm{H})$, 2.92 (q, $4 \mathrm{H}, J=7 \mathrm{~Hz}), 3.20-3.43(\mathrm{~m}, 8 \mathrm{H}), 3.89-4.02(\mathrm{~m}, 4 \mathrm{H}), 3.95$ (s, $6 \mathrm{H}), 9.13$ (s, $2 \mathrm{H}), 9.20$ (s, $2 \mathrm{H}) ;{ }^{13} \mathrm{C} \mathrm{NMR}\left(\mathrm{CDCl}_{3}, \delta\right)$ 14.0, 14.1, 15.3, 15.8, 22.1, 23.0, 23.6, 23.7, 28.7, 30.3, 33.5, 34.0, 52.2, 120.0, 122.6, 126.2, 127.5, 127.7, 128.3, 129.6, 133.9, 134.6, 137.6, 138.2, 169.7; HRMS calcd for $\mathrm{C}_{50} \mathrm{H}_{66} \mathrm{O}_{4}$ : 730.4961, found: 730.4963; Anal. calcd for $\mathrm{C}_{50} \mathrm{H}_{66} \mathrm{O}_{4}$ : C, 82.15; H, 9.10; found: C, 81.97; H, 9.14 . 


\section{Preparation of 1,4-dibutyl-2,3-bis(methoxycarbonyl)-9,10-dihydroanthracene (4d) from 3d}

The compound $\mathbf{4 d}$ was prepared in 59\% yield from diyne $\mathbf{3 d}$ by the same way as described for $\mathbf{4 b}$. 4d: colorless solid; $\mathrm{mp}=85.0-87.0{ }^{\circ} \mathrm{C} ;{ }^{1} \mathrm{H} \mathrm{NMR}\left(\mathrm{CDCl}_{3}, \delta\right) 0.97$ (t, $\left.6 \mathrm{H}, J=7 \mathrm{~Hz}\right), 1.36-1.63(\mathrm{~m}, 8$ $\mathrm{H}), 2.71-2.82(\mathrm{~m}, 4 \mathrm{H}), 3.84(\mathrm{~s}, 6 \mathrm{H}), 3.92(\mathrm{~s}, 4 \mathrm{H}), 7.18-7.35(\mathrm{~m}, 4 \mathrm{H}) ;{ }^{13} \mathrm{C} \mathrm{NMR}\left(\mathrm{CDCl}_{3}, \delta\right) 13.9$, 23.0, 30.3, 32.9, 33.0, 52.3, 126.4, 127.0, 130.2, 135.6, 136.3, 138.5, 169.5; HRMS Calcd for $\mathrm{C}_{26} \mathrm{H}_{32} \mathrm{O}_{4}$ : 408.2301; Found: 408.2304.

\section{Preparation of 1,4-dibutyl -2,3-bis(methoxycarbonyl)anthracene (5d) from 4d}

The compound $\mathbf{5 d}$ was prepared in quantitative yield from dihydroanthracene $\mathbf{4 d}$ by the same way as described for $\mathbf{5 b}$.

5d: pale-yellow solid; mp $=91.5-92.0{ }^{\circ} \mathrm{C} ;{ }^{1} \mathrm{H} \mathrm{NMR}\left(\mathrm{CDCl}_{3}, \delta\right) 1.01(\mathrm{t}, 6 \mathrm{H}, J=7 \mathrm{~Hz}), 1.55$ (tq, $4 \mathrm{H}$, $J=7,7 \mathrm{~Hz}$ ), 1.73-1.88 (m, $4 \mathrm{H}), 3.17-3.29$ (m, $4 \mathrm{H}), 3.93$ (s, $6 \mathrm{H}), 7.49-7.58$ (m, $2 \mathrm{H}), 8.00-8.08$ (m, $2 \mathrm{H}), 8.68(\mathrm{~s}, 2 \mathrm{H}) ;{ }^{13} \mathrm{C} \mathrm{NMR}\left(\mathrm{CDCl}_{3}, \delta\right)$ 14.0, 23.3, 30.2, 33.5, 52.4, 125.0, 126.5, 127.1, 128.4, 130.1, 132.1, 137.6, 170.0; Anal. calcd for $\mathrm{C}_{26} \mathrm{H}_{30} \mathrm{O}_{4}$ : C, 76.82; H, 7.44; Found: C, 76.83; H, 7.45.

\section{Preparation of 1,4-dibutyl-2,3-bis(bromomethyl)anthracene (6d) from 5d}

The compound $\mathbf{6 d}$ was prepared in $86 \%$ yield from anthracene $\mathbf{5 d}$ by the same way as described for 6b.

6d: yellow powder; $\mathrm{mp}=143.7-144.0{ }^{\circ} \mathrm{C} ;{ }^{1} \mathrm{H} \mathrm{NMR}\left(\mathrm{CDCl}_{3}, \delta\right) 1.10(\mathrm{t}, 6 \mathrm{H}, J=7 \mathrm{~Hz}), 1.68(\mathrm{tq}, 4 \mathrm{H}$, $J=7,7 \mathrm{~Hz}$ ), 1.77-1.94 (m, $4 \mathrm{H}), 3.23-3.48$ (m, $4 \mathrm{H}), 5.00$ (s, $4 \mathrm{H})$, 7.46-7.56 (m, $2 \mathrm{H})$, 7.98-8.08 (m, $2 \mathrm{H}), 8.61(\mathrm{~s}, 2 \mathrm{H}) ;{ }^{13} \mathrm{C} \mathrm{NMR}\left(\mathrm{CDCl}_{3}, \delta\right)$ 14.0, 23.5, 29.0, 29.5, 33.1, 124.2, 126.0, 128.4, 129.8, 130.5, 131.8, 138.8; Anal. Calcd for $\mathrm{C}_{24} \mathrm{H}_{28} \mathrm{Br}_{2}$ : C, 60.52; H, 5.93; Found: C, 60.66; H, 6.01

\section{Preparation of 1,4-dibutyl-2,3-bis(2-heptynyl)anthracene (7d) from 6d}

The compound $\mathbf{7 d}$ was prepared in $68 \%$ yield from dibromide $\mathbf{6 d}$ and 1 -hexyne by the same way as described for $\mathbf{7 b}$.

7d: yellow powder; $\mathrm{mp}=114.8-115.8{ }^{\circ} \mathrm{C} ;{ }^{1} \mathrm{H} \mathrm{NMR}\left(\mathrm{CDCl}_{3}, \delta\right) 0.87(\mathrm{t}, 6 \mathrm{H}, J=7 \mathrm{~Hz}), 1.06(\mathrm{t}, 6 \mathrm{H}$, $J=7 \mathrm{~Hz}), 1.28-1.51(\mathrm{~m}, 8 \mathrm{H}), 1.57-1.71(\mathrm{~m}, 4 \mathrm{H}), 1.71-1.85(\mathrm{~m}, 4 \mathrm{H}), 2.08-2.18(\mathrm{~m}, 4 \mathrm{H}), 3.22-3.32$ $(\mathrm{m}, 4 \mathrm{H}), 3.86(\mathrm{t}, 4 \mathrm{H}, J=2 \mathrm{~Hz}), 7.40-7.47$ (m, $2 \mathrm{H}), 7.96-8.04(\mathrm{~m}, 2 \mathrm{H}), 8.57(\mathrm{~s}, 2 \mathrm{H}) ;{ }^{13} \mathrm{C} \mathrm{NMR}$ $\left(\mathrm{CDCl}_{3}, \delta\right)$ 13.6, 14.1, 18.6, 20.1, 21.9, 23.5, 29.3, 31.0, 32.9, 78.1, 81.2, 123.2, 125.0, 128.3, 130.5, 131.0, 131.9, 134.7; HRMS Calcd for $\mathrm{C}_{36} \mathrm{H}_{46}$ : 478.3600; Found: 478.3591.

\section{Preparation of 1,4,6,13-tetrabutyl-2,3-bis(methoxycarbonyl)-5,14-dihydropentacene (8d) from} 7d

The compound $\mathbf{8 d}$ was prepared by the same way as described for $\mathbf{8 b}$ in $51 \%$ yield from diyne $\mathbf{7 d}$.

8d: pale-yellow crystals; $\mathrm{mp}=147.0-147.9^{\circ} \mathrm{C} ;{ }^{1} \mathrm{H} \mathrm{NMR}\left(\mathrm{CDCl}_{3}, \delta\right) 1.03$ (t, $\left.6 \mathrm{H}, J=7 \mathrm{~Hz}\right), 1.09$ (t, $6 \mathrm{H}, J=7 \mathrm{~Hz}), 1.45-1.85(\mathrm{~m}, 16 \mathrm{H}), 2.81-2.93$ (m, $4 \mathrm{H}), 3.29-3.42(\mathrm{~m}, 4 \mathrm{H}), 3.85$ (s, $6 \mathrm{H}), 4.14$ (s, 4 $\mathrm{H}), 7.38-7.47(\mathrm{~m}, 2 \mathrm{H}), 7.95-8.05(\mathrm{~m}, 2 \mathrm{H}), 8.59$ (s, $2 \mathrm{H}) ;{ }^{13} \mathrm{C} \mathrm{NMR}\left(\mathrm{CDCl}_{3}, \delta\right)$ 13.9, 14.0, 23.2, 
$23.5,28.7,30.4,30.5,33.0,33.4,52.2,123.0,125.0,128.2,130.1,130.3,130.9,131.8,131.9,135.2$, 139.5, 169.5; Anal. Calcd for $\mathrm{C}_{42} \mathrm{H}_{5} \mathrm{O}_{4}$ : C, 81.25; H, 8.44; Found: C, 81.23; H, 8.60.

\section{Preparation of 1,4,6,13-tetrabutyl-2,3-bis(methoxycarbonyl) pentacene (9d) from 8d}

The compound 9d was prepared in $48 \%$ yield from dihydropentacene $8 \mathbf{c}$ by the same way as described for $\mathbf{9 b}$.

9d: dark-blue crystals; $\mathrm{mp}=172.9-173.6{ }^{\circ} \mathrm{C} ;{ }^{1} \mathrm{H} \mathrm{NMR}\left(\mathrm{CDCl}_{3}, \delta\right) 1.05$ (t, $\left.6 \mathrm{H}, J=7 \mathrm{~Hz}\right), 1.10$ (t, 6 $\mathrm{H}, J=7 \mathrm{~Hz}), 1.53-1.80(\mathrm{~m}, 8 \mathrm{H}), 1.83-2.05(\mathrm{~m}, 8 \mathrm{H}), 3.20-3.31(\mathrm{~m}, 4 \mathrm{H}), 3.86-3.97(\mathrm{~m}, 4 \mathrm{H}), 3.94$ $(\mathrm{s}, 6 \mathrm{H}), 7.31-7.40(\mathrm{~m}, 2 \mathrm{H}), 7.93-8.02(\mathrm{~m}, 2 \mathrm{H}), 8.91(\mathrm{~s}, 2 \mathrm{H}), 9.20(\mathrm{~s}, 2 \mathrm{H}) ;{ }^{13} \mathrm{C} \mathrm{NMR}\left(\mathrm{CDCl}_{3}, \delta\right)$ $14.0,14.2,23.5,23.8,28.9,30.4,33.5,34.0,52.3,122.9,123.8,125.4,126.5,127.6,128.5,128.6$, 128.7, 131.2, 134.5, 138.2, 169.6; HRMS calcd for $\mathrm{C}_{42} \mathrm{H}_{50} \mathrm{O}_{4}$ : 618.3709, found: 618.3709; Anal. calcd for $\mathrm{C}_{42} \mathrm{H}_{50} \mathrm{O}_{4}$ : C, 81.51; H, 8.14; found: C, 81.43; H, 8.20.

\section{Preparation of 1,4-bis(trimethylsilyl)-2,3-bis(methoxycarbonyl)anthracene (5e) from 4e}

The compound $\mathbf{5 e}$ was prepared in $78 \%$ yield from dihydroanthracene $4 \mathbf{e}$ by the same way as described for $\mathbf{5 b}$.

5e: ${ }^{1} \mathrm{H}$ NMR $\left(\mathrm{CDCl}_{3}, \delta\right) 0.55$ (s, $\left.18 \mathrm{H}\right), 3.90$ (s, $\left.6 \mathrm{H}\right), 7.52-7.55(\mathrm{~m}, 2 \mathrm{H}), 7.98-8.02(\mathrm{~m}, 2 \mathrm{H}), 8.82$ (s, $2 \mathrm{H}) ;{ }^{13} \mathrm{C} \mathrm{NMR}\left(\mathrm{CDCl}_{3}, \delta\right) 2.2,52.5,126.4,128.1,129.1,130.9$, 133.4, 135.6, 139.1, 170.7; HRMS calcd for $\mathrm{C}_{24} \mathrm{H}_{30} \mathrm{O}_{4} \mathrm{Si}_{2}$ : 438.1683, found: 438.1678 .

\section{Preparation of 1,4-dibutyl-2,3-bis(methoxycarbonyl)-5,14-dihydropentacene (8e) from 7e}

The compound $\mathbf{8 e}$ was prepared in $40 \%$ yield from diyne $7 \mathbf{e}$ by the same way as described for $\mathbf{8 b}$.

8e: pale yellow powder; ${ }^{1} \mathrm{H}$ NMR $\left(\mathrm{CDCl}_{3}, \delta\right) 0.99(\mathrm{t}, J=7.2 \mathrm{~Hz}, 6 \mathrm{H}), 1.48(\mathrm{~m}, 8 \mathrm{H}), 2.83(\mathrm{t}, J=7.5$ $\mathrm{Hz}, 4 \mathrm{H}), 3.83$ (s, $6 \mathrm{H}), 4.11$ (s, $4 \mathrm{H}), 7.44$ (dd, $J=6.6,3.3 \mathrm{~Hz}, 2 \mathrm{H}), 7.92$ (dd, $J=6.6,3.3 \mathrm{~Hz}, 2 \mathrm{H})$, $7.98(\mathrm{~s}, 2 \mathrm{H}), 8.38(\mathrm{~s}, 2 \mathrm{H}) ;{ }^{13} \mathrm{C} \mathrm{NMR}\left(\mathrm{CDCl}_{3}, \delta\right)$ 13.9, 23.0, 30.3, 33.1, 33.6, 52.2, 124.4, 125.1, 125.4, 128.0, 130.4, 131.0, 131.5, 135.1, 135.4, 138.9, 169.4; HRMS calcd. for $\mathrm{C}_{34} \mathrm{H}_{36} \mathrm{O}_{4}: 508.2613$, found: 508.2611 .

\section{1,4-Bis(trimethylsilyl)-2,3-bis(methoxycarbonyl)-5,7,12,14-tetrahydropentacene (11a) from 10}

The compound $\mathbf{1 1 a}$ was prepared in $41 \%$ yield from diyne $\mathbf{1 0}$ by the same way as described for $\mathbf{4 e .}$ 11a: colorless solid; ${ }^{1} \mathrm{H}$ NMR $\left(\mathrm{CDCl}_{3}, \delta\right) 0.31(\mathrm{~s}, 18 \mathrm{H}), 3.69(\mathrm{~s}, 6 \mathrm{H}), 3.82(\mathrm{~s}, 4 \mathrm{H}), 3.87(\mathrm{~s}, 4 \mathrm{H})$, 7.06-7.09 (m, $2 \mathrm{H}), 7.11$ (s, $2 \mathrm{H}), 7.15-7.19$ (m, $2 \mathrm{H}) ;{ }^{13} \mathrm{C} \mathrm{NMR}\left(\mathrm{CDCl}_{3}, \delta\right)$ 1.8, 35.9, 38.1, 52.3, 125.2, 126.0, 127.3, 134.8, 135.0, 135.5, 135.8, 136.7, 146.0, 170.5; HRMS (FAB): calcd for $\mathrm{C}_{32} \mathrm{H}_{38} \mathrm{O}_{4} \mathrm{Si}_{2} \mathrm{Na}\left(\mathrm{M}+\mathrm{Na}^{+}\right)$565.2201, found: 565.2209.

\section{Preparation of 1,4-bis(trimethylsilyl)-2,3-bis(ethoxycarbonyl)-5,7,12,14-tetrahydropentacene (11b) from 10 \\ The compound 11b was prepared in 48\% isolated yield from diyne $\mathbf{1 0}$ and diethyl acetylenedicarboxylate by the same way as described for $\mathbf{4 e}$.}


11b: ${ }^{1} \mathrm{H}$ NMR $\left(\mathrm{CDCl}_{3}, \delta\right) 0.42(\mathrm{~s}, 18 \mathrm{H}), 1.33(\mathrm{t}, J=7.2 \mathrm{~Hz}, 6 \mathrm{H}), 3.92(\mathrm{~s}, 4 \mathrm{H}), 3.96(\mathrm{~s}, 4 \mathrm{H}), 4.25$ $(\mathrm{q}, J=7.2 \mathrm{~Hz}, 4 \mathrm{H}), 7.16-7.19(\mathrm{~m}, 2 \mathrm{H}), 7.24(\mathrm{~s}, 2 \mathrm{H}), 7.26-7.29(\mathrm{~m}, 2 \mathrm{H}) ;{ }^{13} \mathrm{C} \mathrm{NMR}\left(\mathrm{CDCl}_{3}, \delta\right) 1.9$, 13.8, 35.1, 38.1, 61.5, 125.2, 126.0, 127.4, 134.8, 135.1, 135.6, 135.8, 136.7, 145.9, 170.2; HRMS (FAB): calcd for $\mathrm{C}_{34} \mathrm{H}_{42} \mathrm{O}_{4} \mathrm{Si}_{2} \mathrm{Na}\left(\mathrm{M}+\mathrm{Na}^{+}\right)$593.2514, found: 593.2532.

\section{Preparation of 2,3-bis(ethoxycarbonyl)-5,7,12,14-tetrahydropentacene (12b) from 11b}

The compound $\mathbf{1 2 b}$ was prepared in $76 \%$ isolated yield from $\mathbf{1 1 b}$ by the same way as described for dimethyl ester 12a.

12b: ${ }^{1} \mathrm{H} \mathrm{NMR}\left(\mathrm{CDCl}_{3}, \delta\right) 1.44$ (t, $\left.J=7.2 \mathrm{~Hz}, 6 \mathrm{H}\right), 3.85$ (s, $\left.4 \mathrm{H}\right), 3.91$ (s, $\left.4 \mathrm{H}\right), 4.45$ (q, $J=14.1 \mathrm{~Hz}$, $J=7.2 \mathrm{~Hz}, 4 \mathrm{H}), 7.11(\mathrm{~s}, 2 \mathrm{H}), 7.22-7.25(\mathrm{~m}, 2 \mathrm{H}), 7.31-7.34(\mathrm{~m}, 2 \mathrm{H}), 7.65(\mathrm{~s}, 2 \mathrm{H})$; ${ }^{13} \mathrm{C} \mathrm{NMR}$ $\left(\mathrm{CDCl}_{3}, \delta\right)$ 14.0, 35.2, 35.6, 61.4, 125.9, 126.2, 127.2, 127.8, 129.9, 132.6, 134.6, 136.5, 140.1, 167.7; HRMS: calcd for $\mathrm{C}_{28} \mathrm{H}_{26} \mathrm{O}_{4} 426.1831$, found: 426.1834 .

\section{Preparation of 2,3-bis(3-trimethylsilylprop-2-ynyl)naphthalene (14) from 13}

This compound $\mathbf{1 4}$ was prepared from diiodide $\mathbf{1 3}$ by the same method as described for diyne $\mathbf{1 0}$ in $93 \%$ yield.

14: colorless solid; ${ }^{1} \mathrm{H}$ NMR $\left(\mathrm{CDCl}_{3}, \delta\right) 0.37$ (s, $\left.18 \mathrm{H}\right), 3.86(\mathrm{~s}, 4 \mathrm{H}), 7.52-7.55(\mathrm{~m}, 2 \mathrm{H})$, 7.86-7.89 $(\mathrm{m}, 2 \mathrm{H}) 7.98(\mathrm{~s}, 2 \mathrm{H}) ;{ }^{13} \mathrm{C} \mathrm{NMR}\left(\mathrm{CDCl}_{3}, \delta\right)$ 0.1, 24.1, 87.8, 103.6, 125.8, 127.3, 127.4, 132.3, 132.5; HRMS: calcd for $\mathrm{C}_{22} \mathrm{H}_{28} \mathrm{Si}_{2} 348.1730$, found: 348.1737 . 


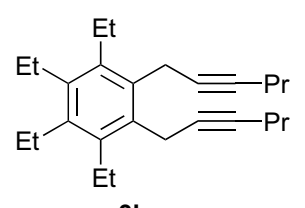

3b

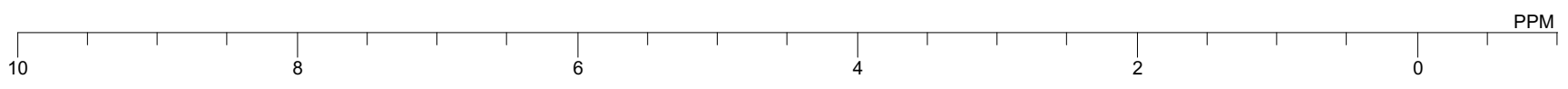

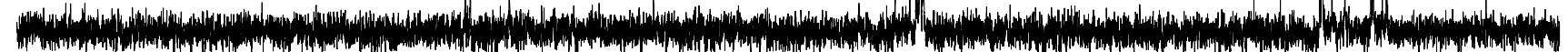

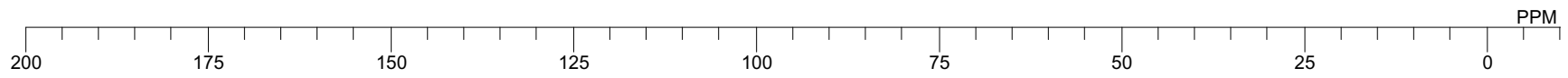



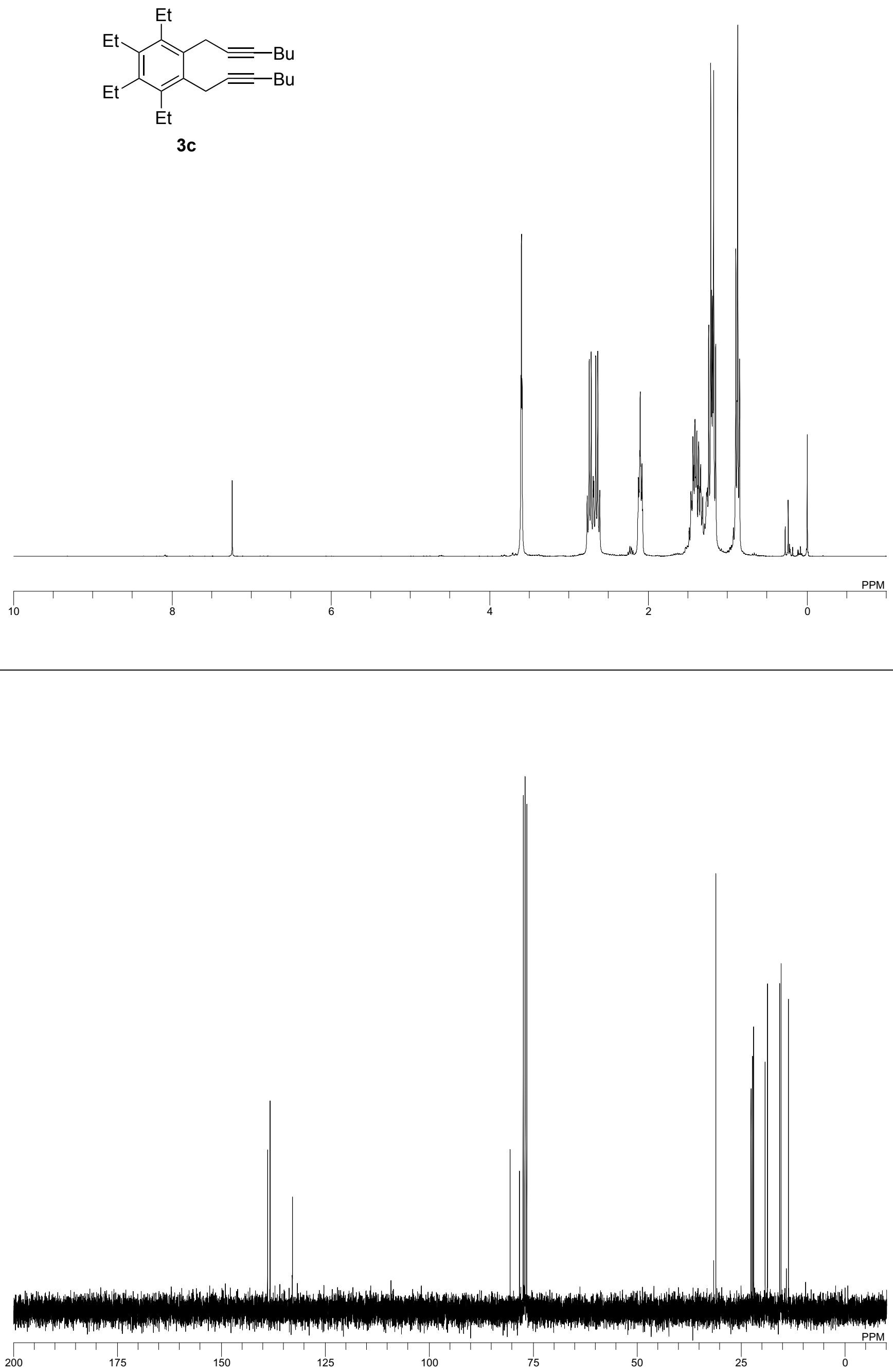


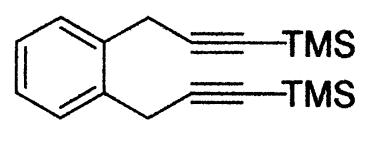

$3 e$
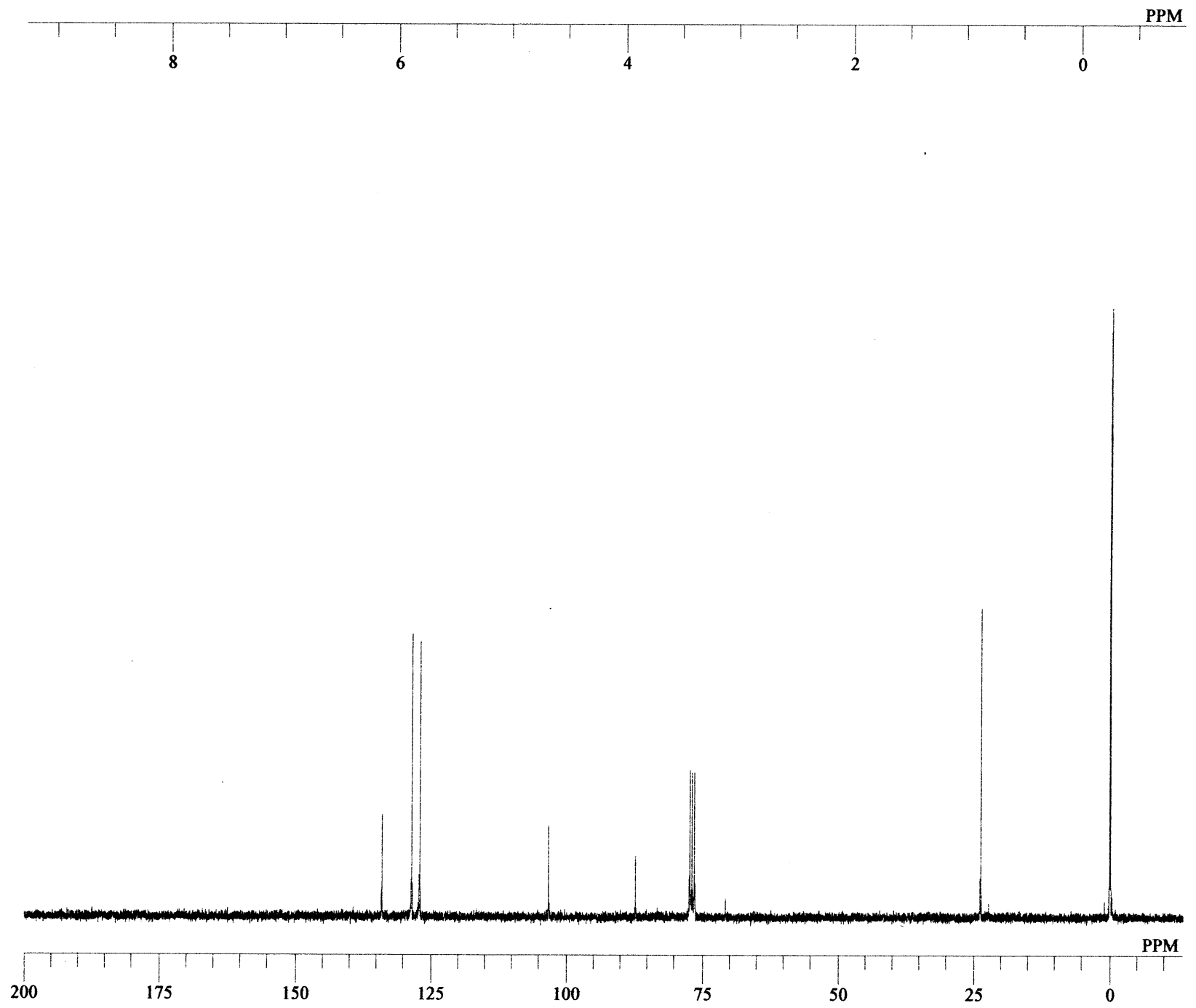
<smiles>CCCC1=C(C(=O)OC)C(C(=O)OC)=C(CCC)C2Cc3c(CC)c(CC)c(CC)c(CC)c3CC12</smiles>

$4 b$

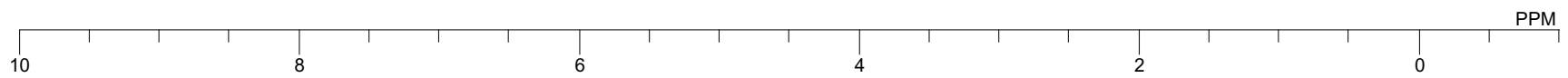



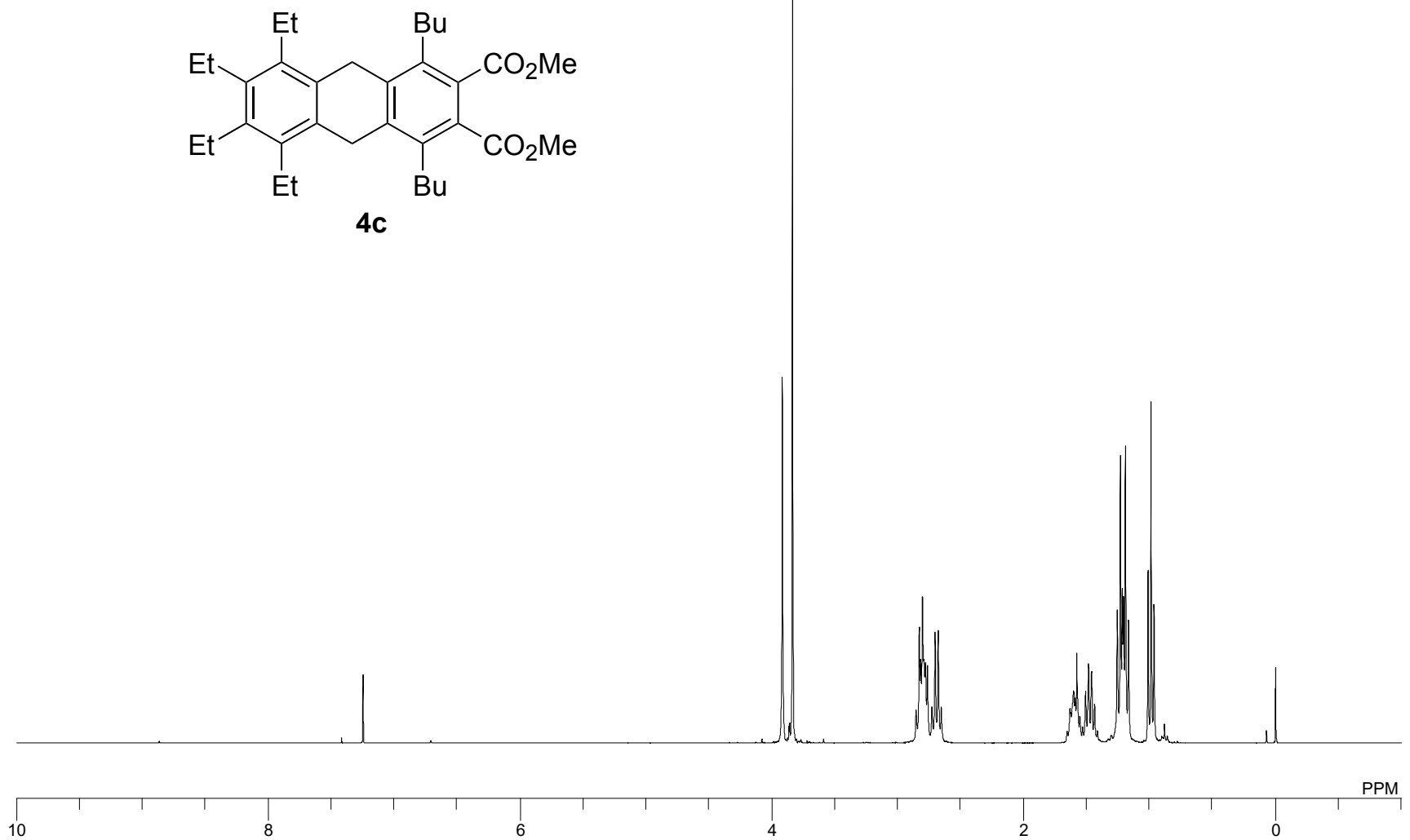

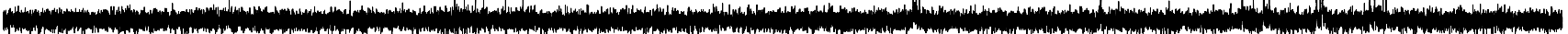

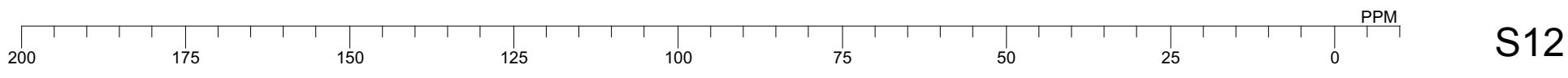




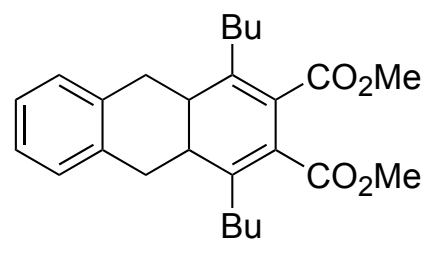

4d

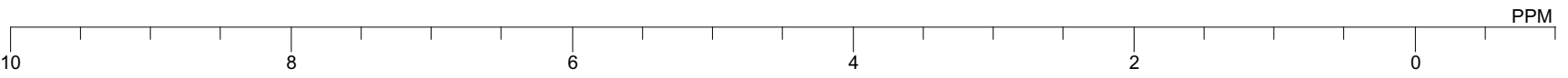

1.

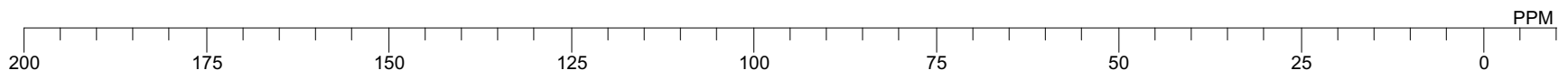



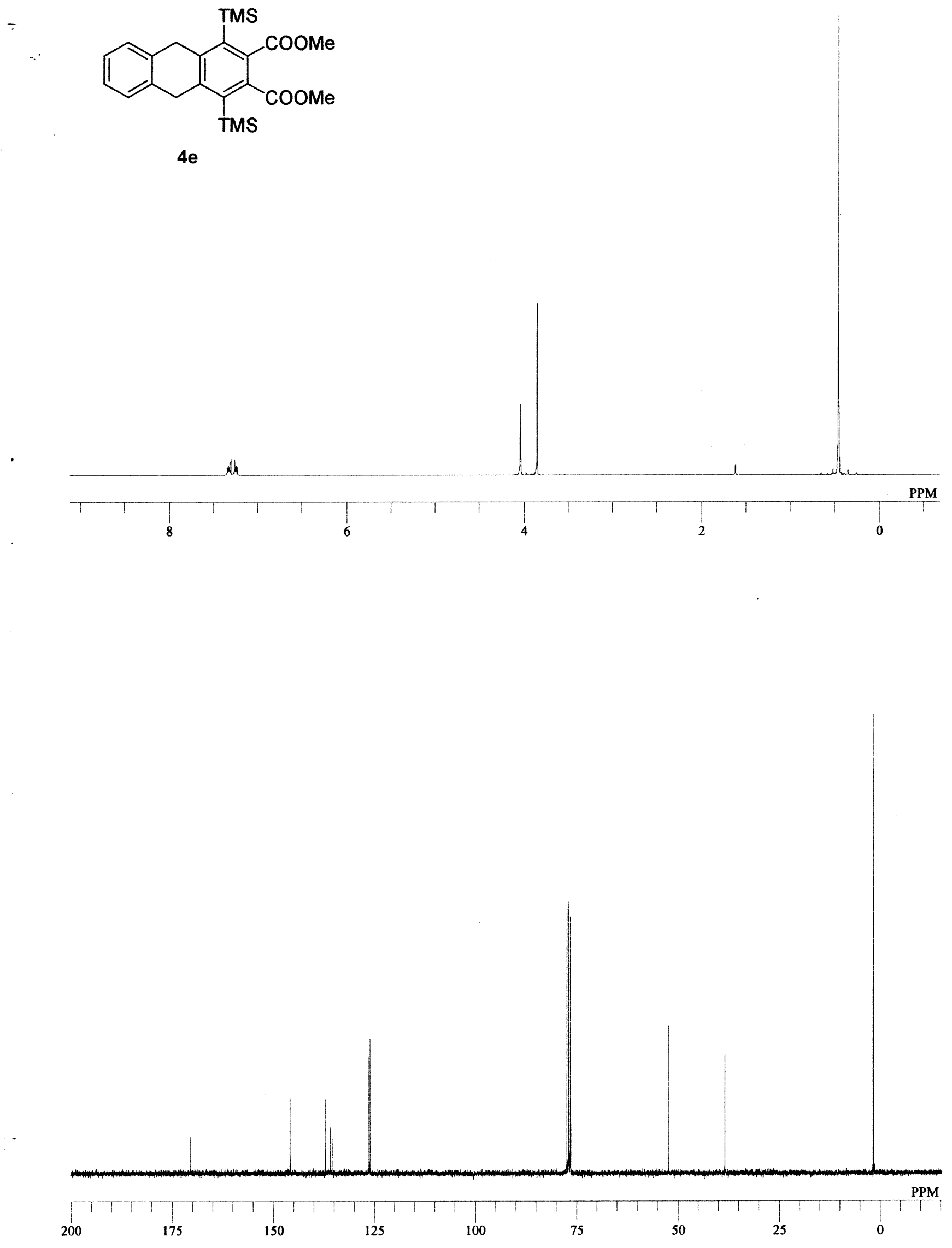

S14 
<smiles></smiles>

$5 a$
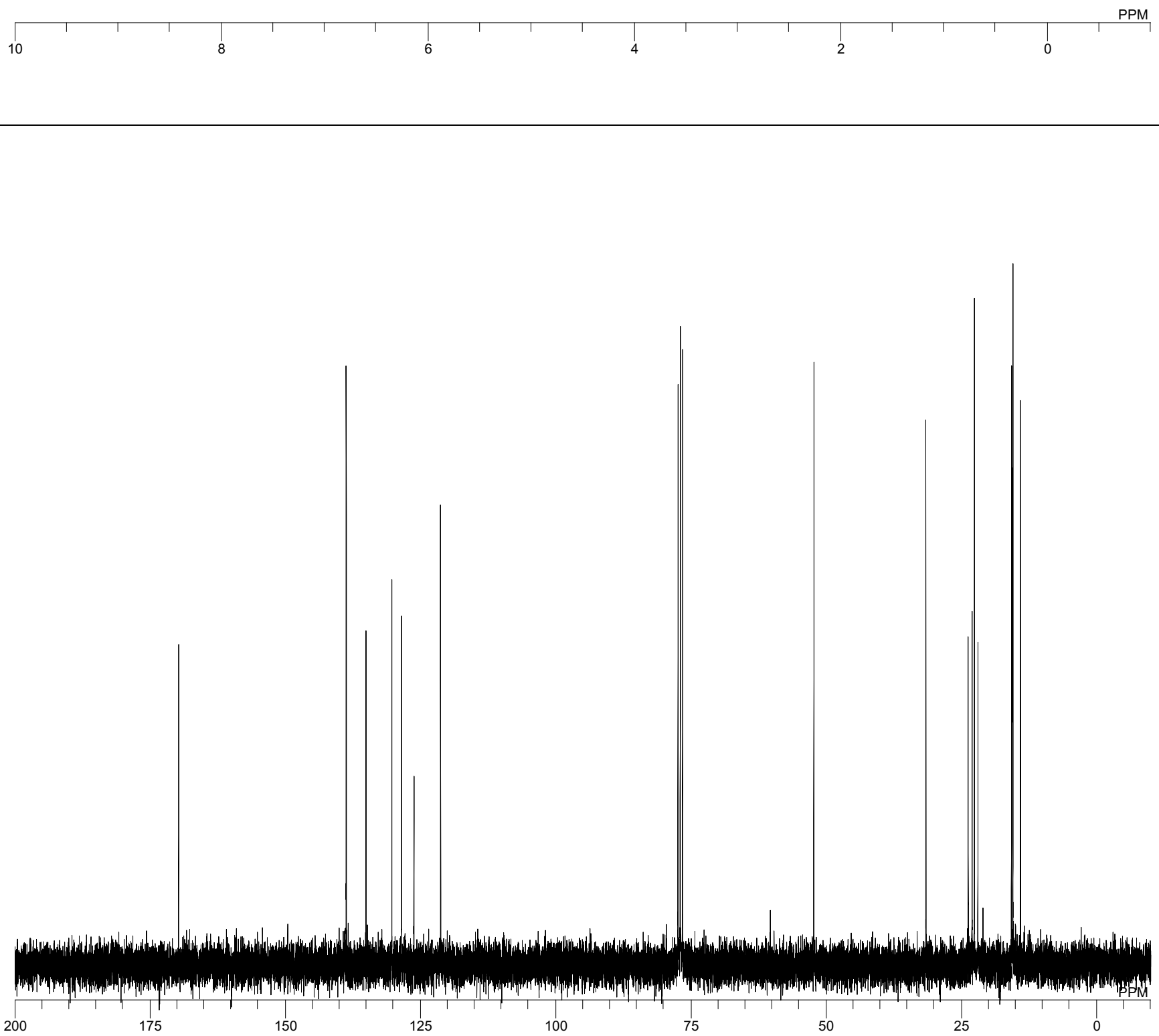


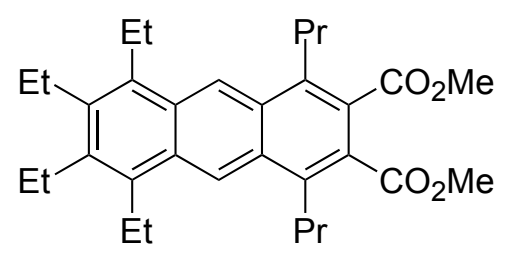

$5 b$
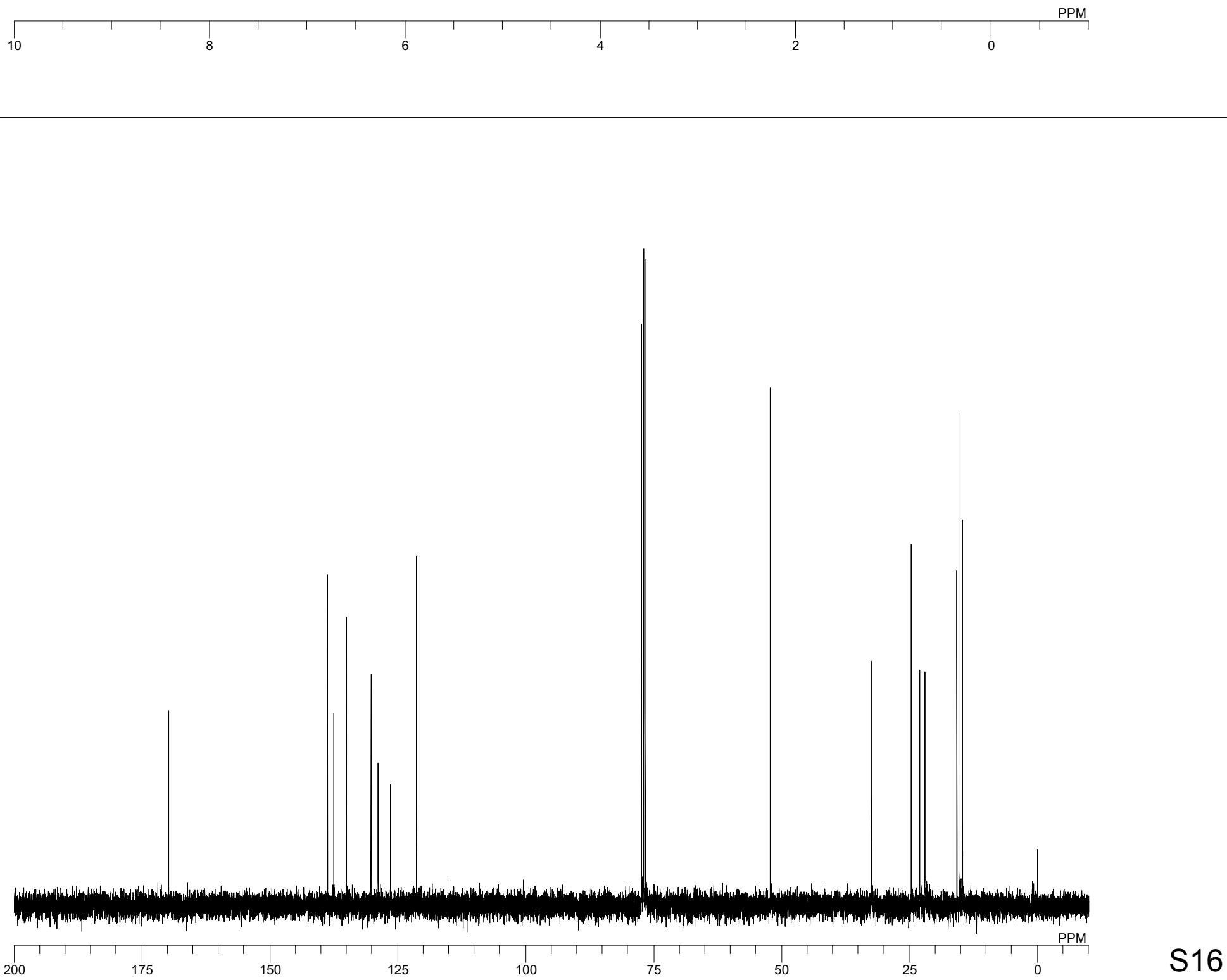
<smiles>CCc1c(CC)c(CC)c2cc3c(C(=O)c4ccccc4)c(C(=O)OC)c(C(C)=O)c(C)c3cc2c1CC</smiles>

5c

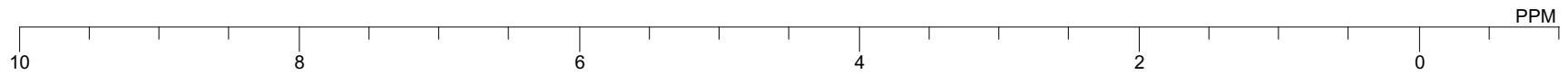

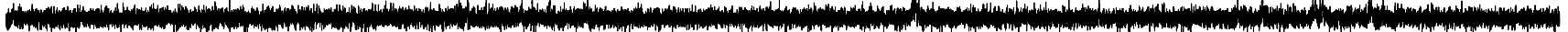

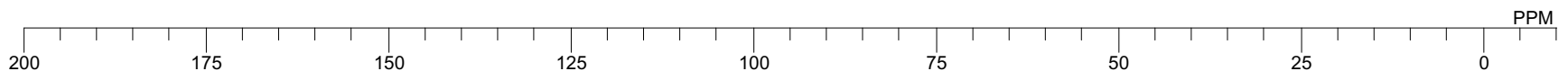




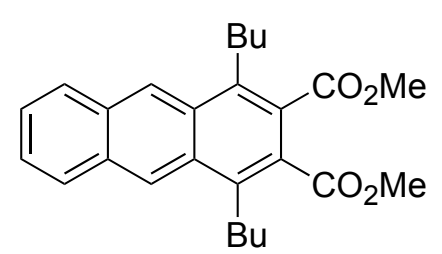

5d

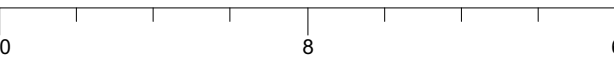

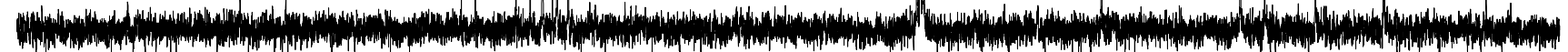

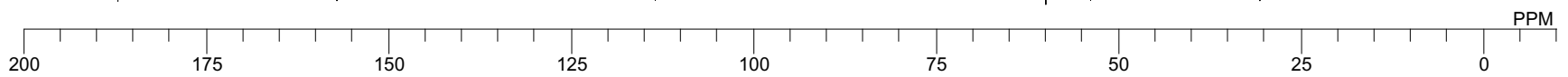


<smiles>COC(=O)c1c(C(C)(C)C)c(C(C)(C)C)c2cc3ccccc3cc2c1C(C)(C)C</smiles>

$5 e$
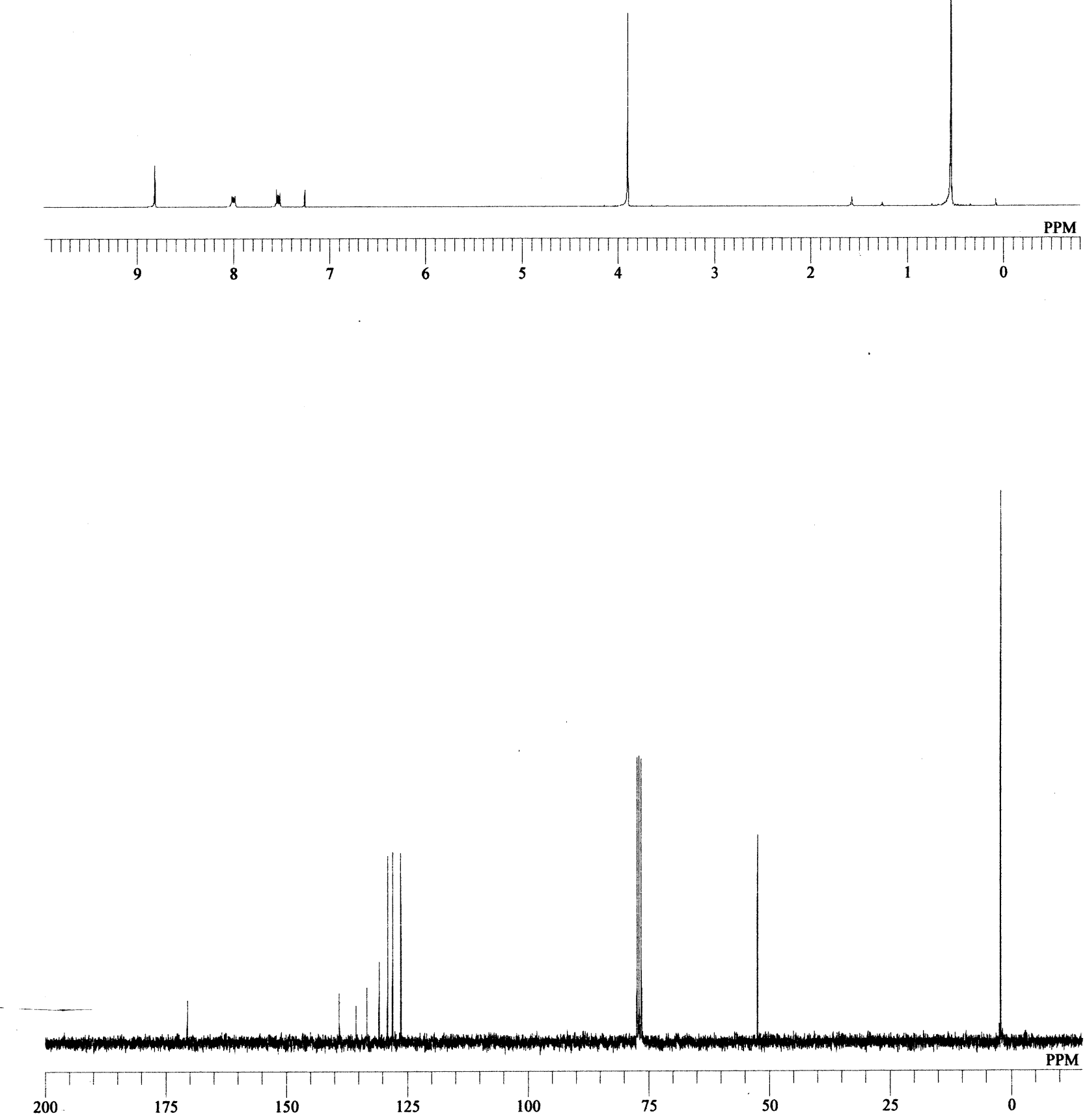
<smiles>CCc1c(CC)c(CC)c2cc3c(CC)c(CBr)c(CBr)c(CC)c3cc2c1CC</smiles>

$6 a$

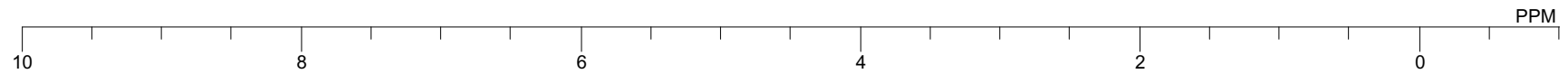

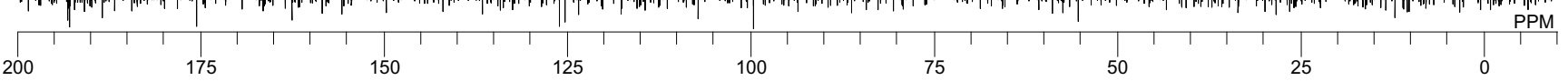


<smiles>CCCc1c(CBr)c(CBr)c(CCC)c2cc3c(CC)c(CC)c(CC)c(CC)c3cc12</smiles>

6b
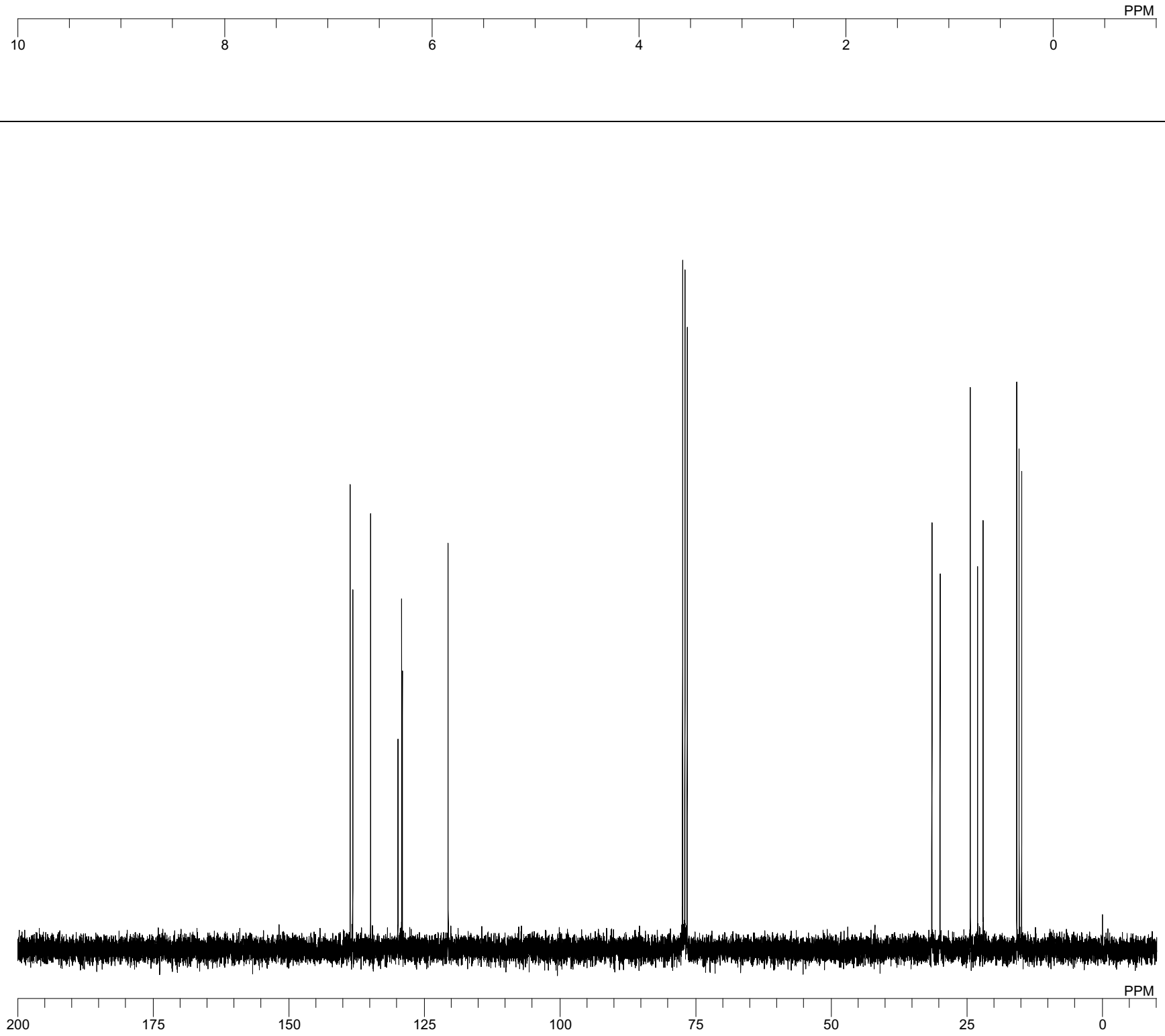
<smiles>CCc1c(CC)c(CC(C)(C)C)c2cc3c(CBr)c(CBr)c(CBr)c(Br)c3cc2c1CC</smiles>
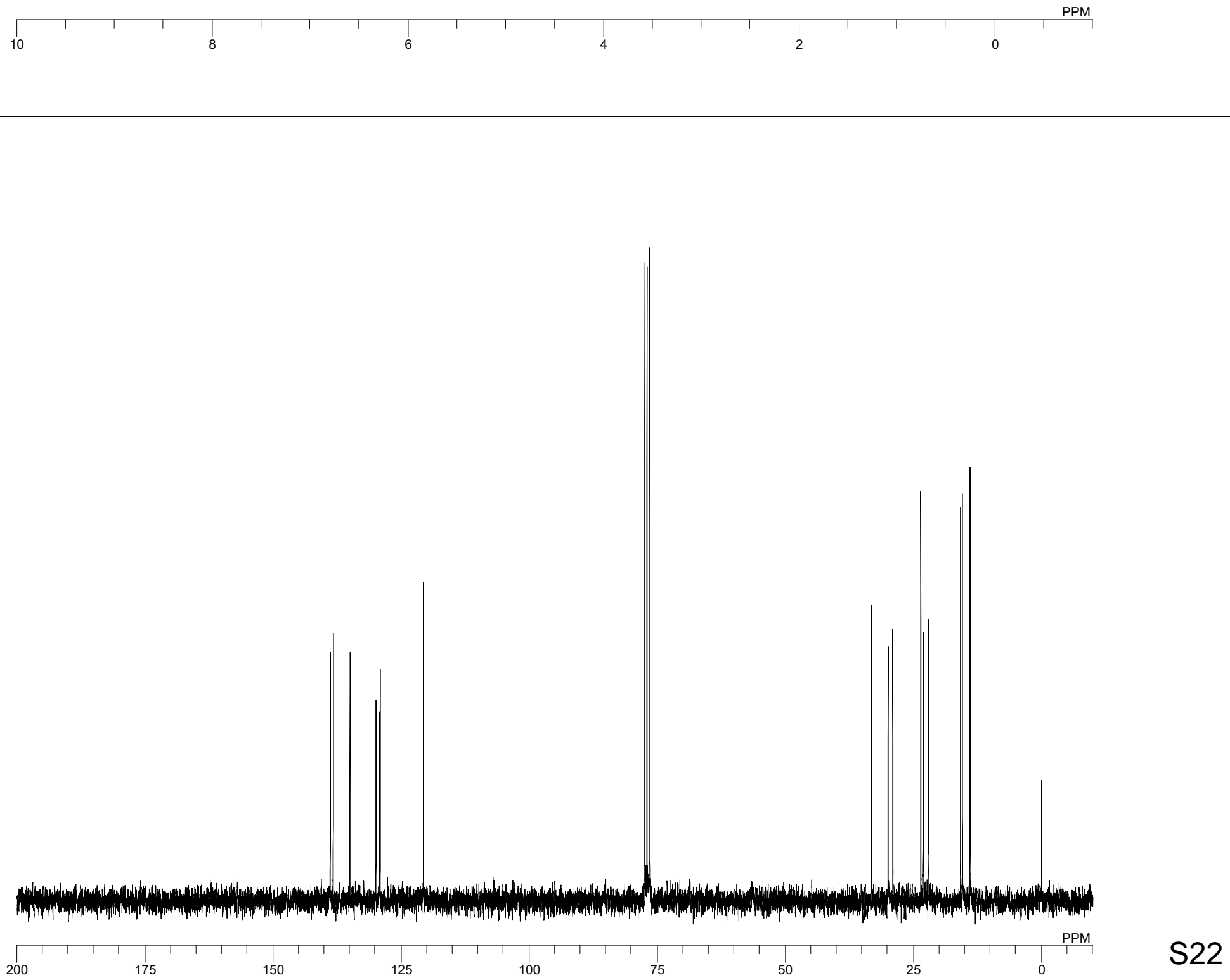


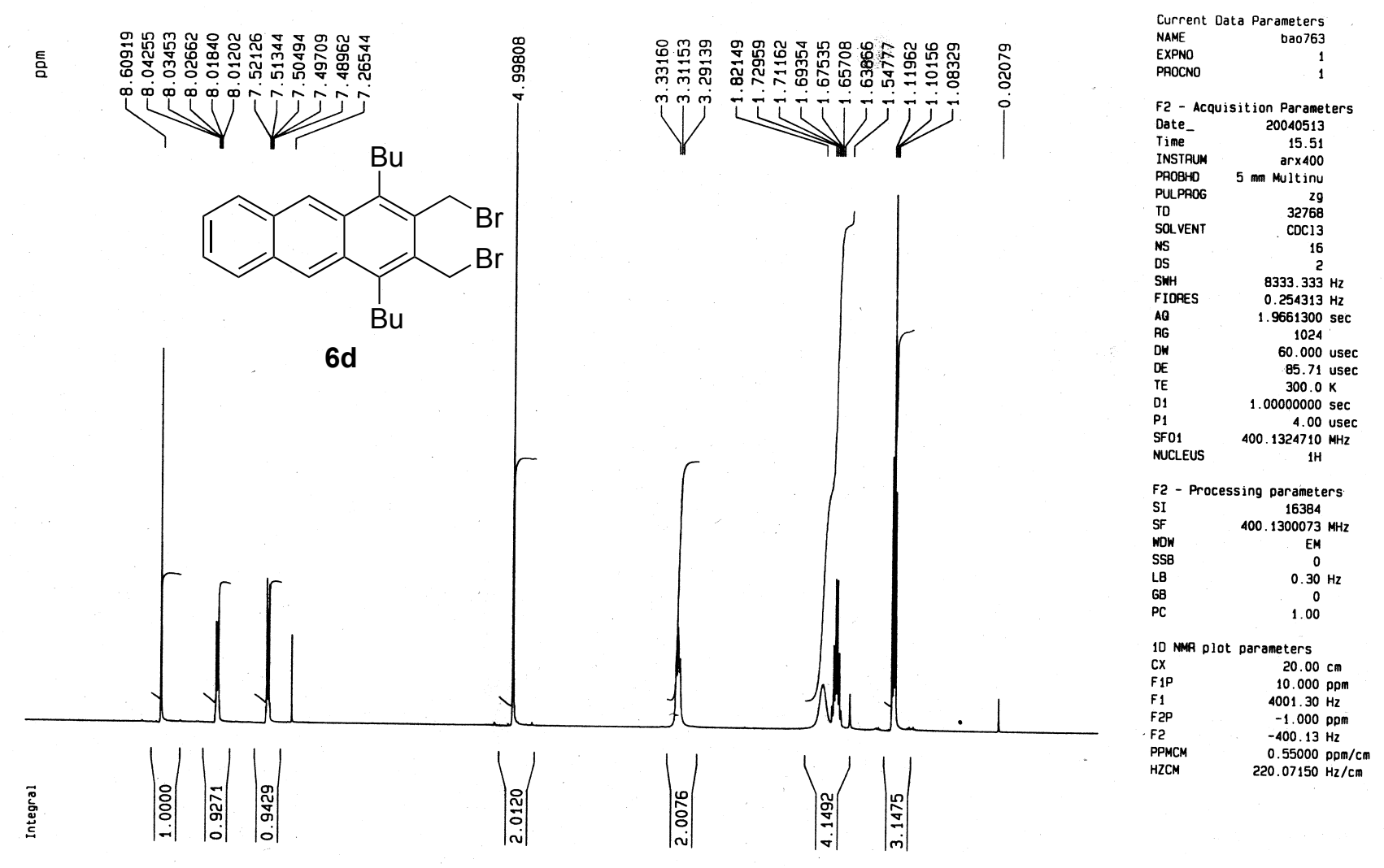

$\mathrm{ppm}_{8}$

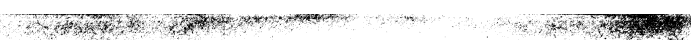

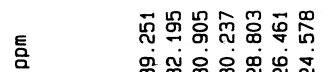

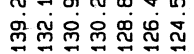

웃윰ำ곡

रहत

V

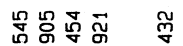

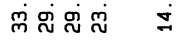
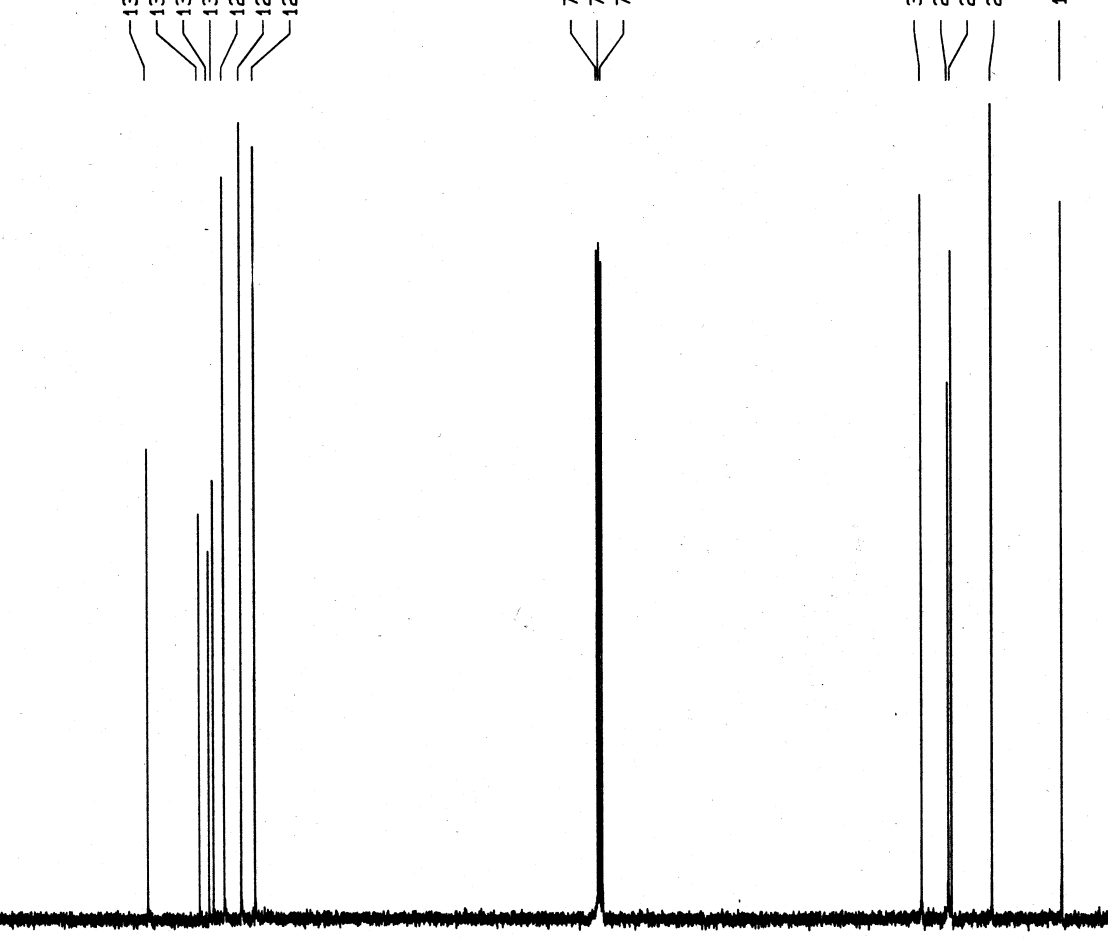

ppm

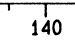

120

${ }_{100}^{1}$

80

60

40 


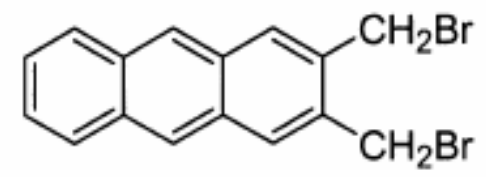

2,3-Bis-bromomethyl-anthracene

$6 e$
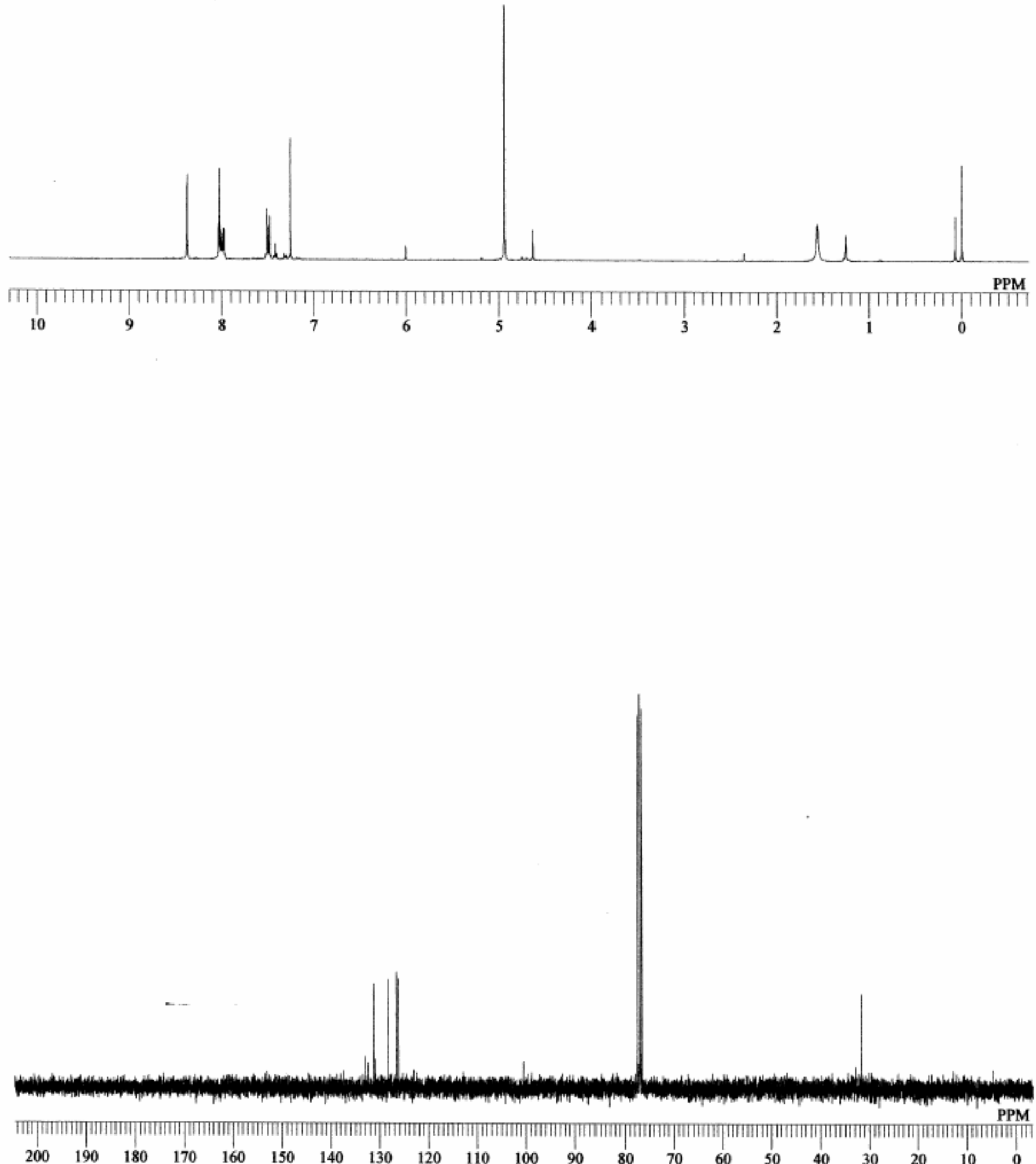


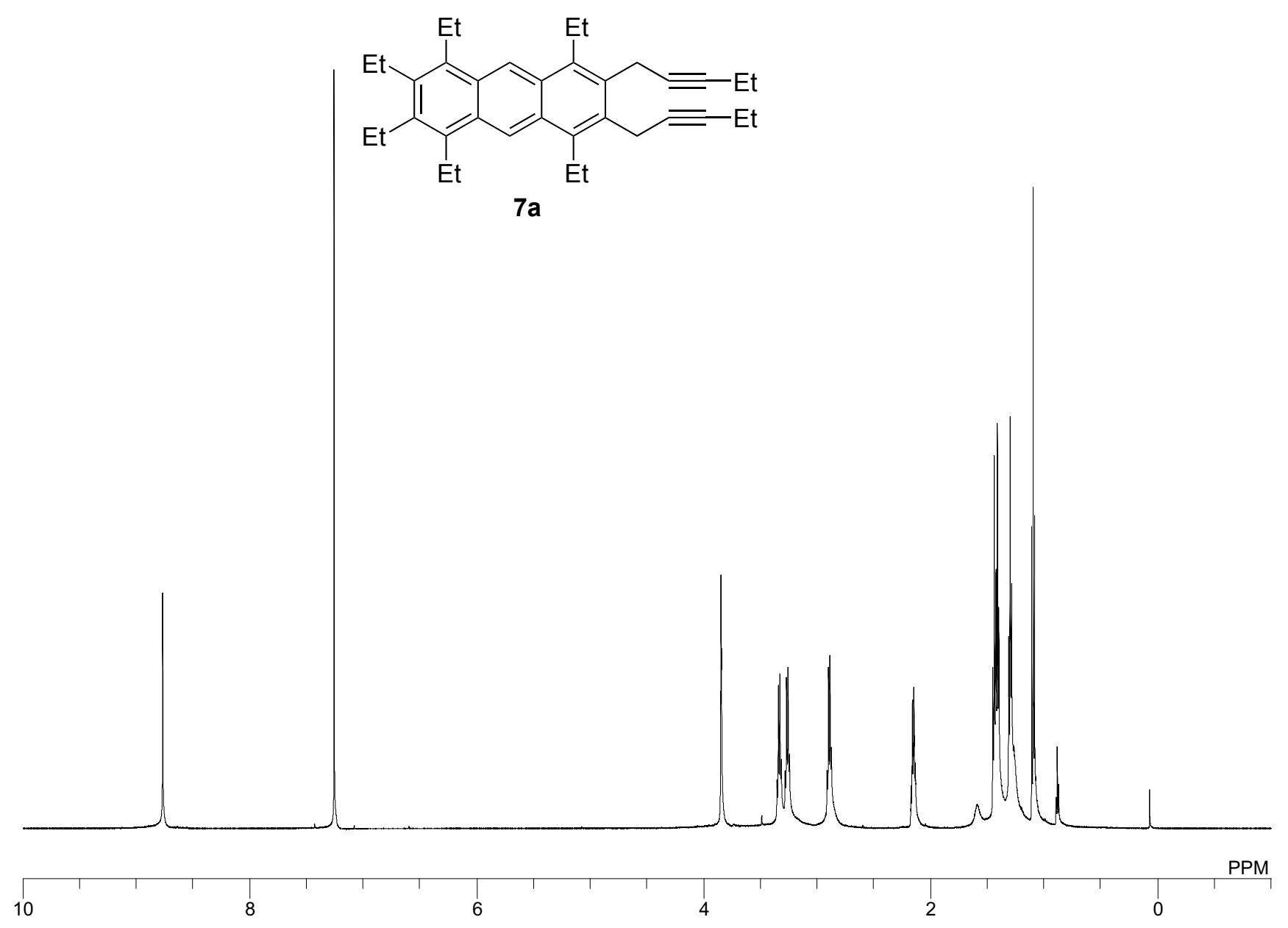


<smiles>CCCC#CCc1c(CC#CPCC)c(CCC)c2cc3c(CC)c(CC)c(CC)c(CC)c3cc2c1CC</smiles>

7b

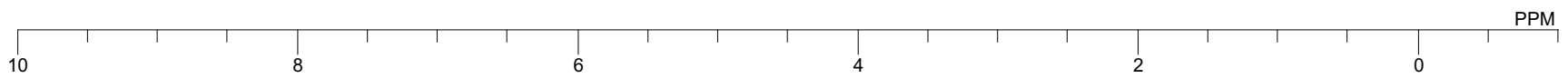




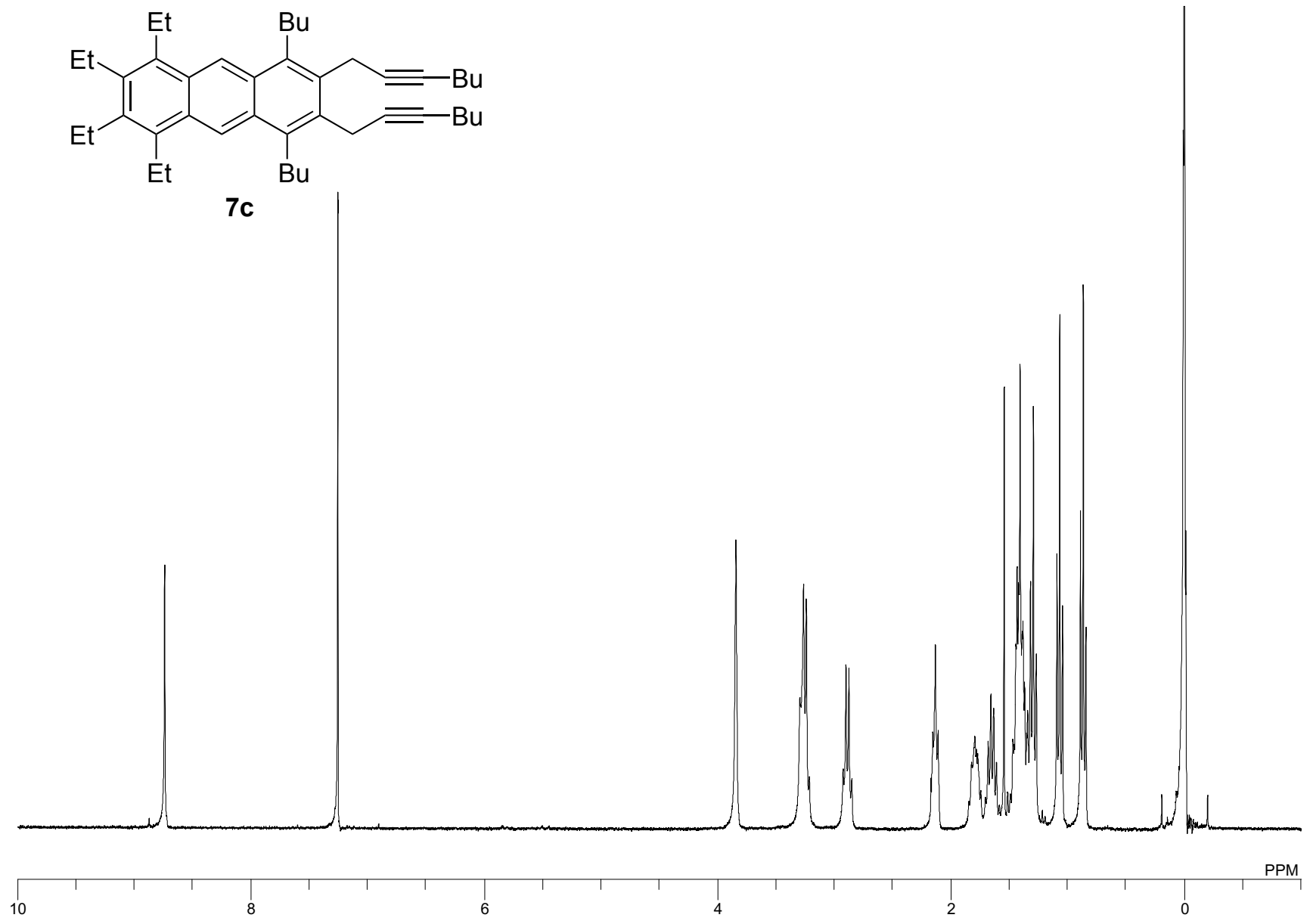

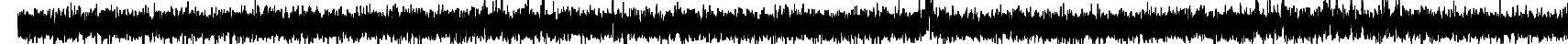

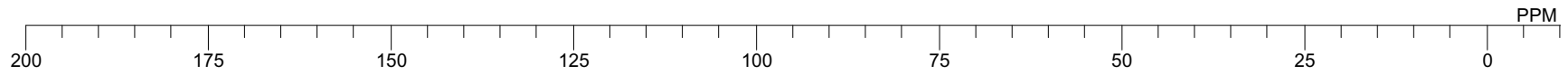


<smiles>CC(C)(C)CC#CCc1c(CC#CBr)c(C(=O)c2ccccc2)c2cc3ccccc3cc2c1C(=O)c1ccccc1</smiles>

7d
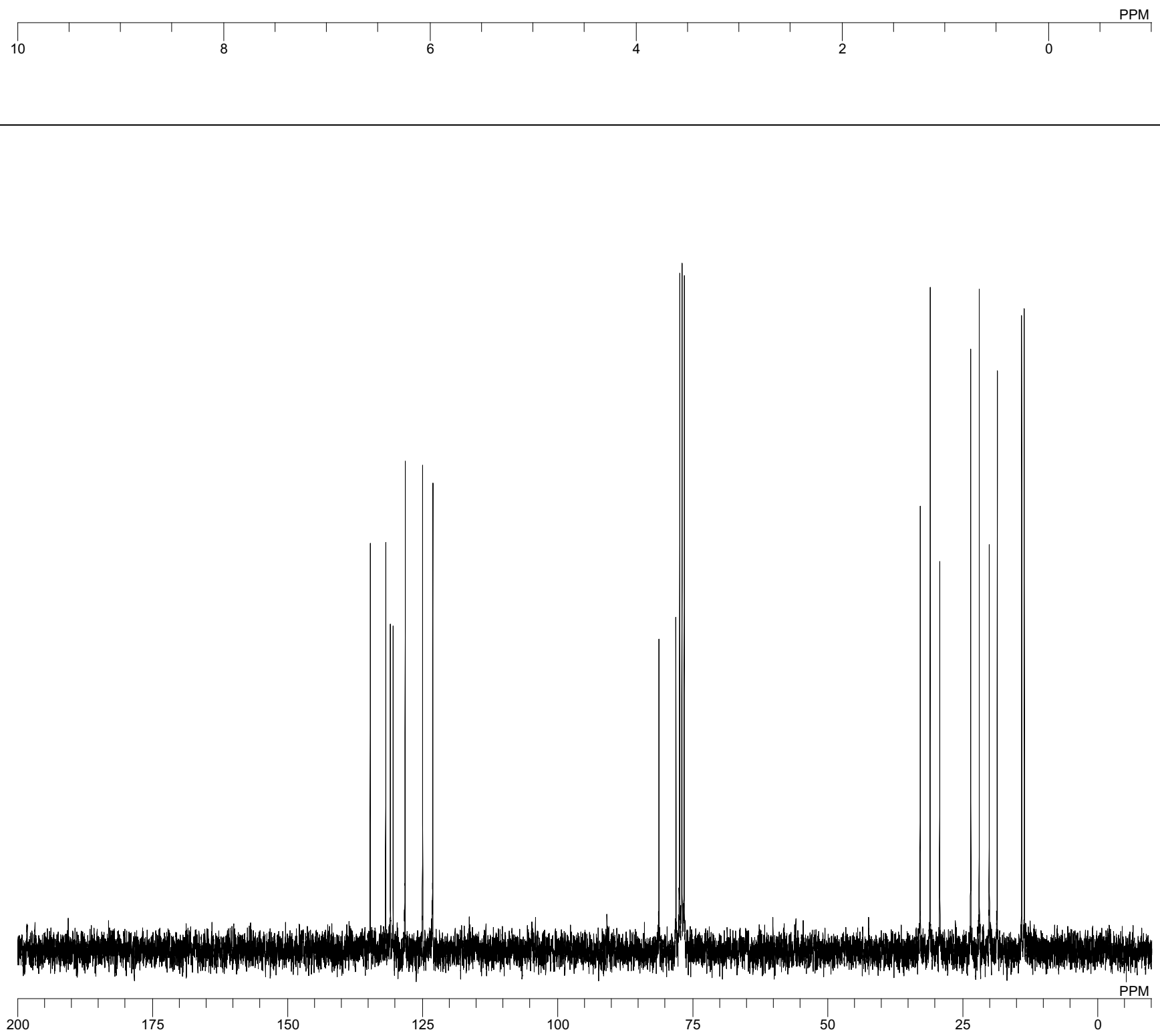


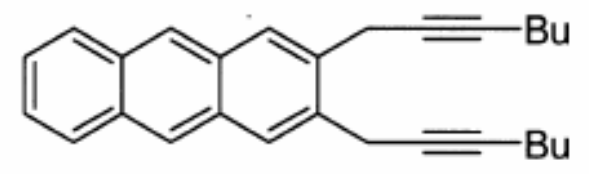

2,3-Di-hept-2-ynyl-anthracene

$7 e$
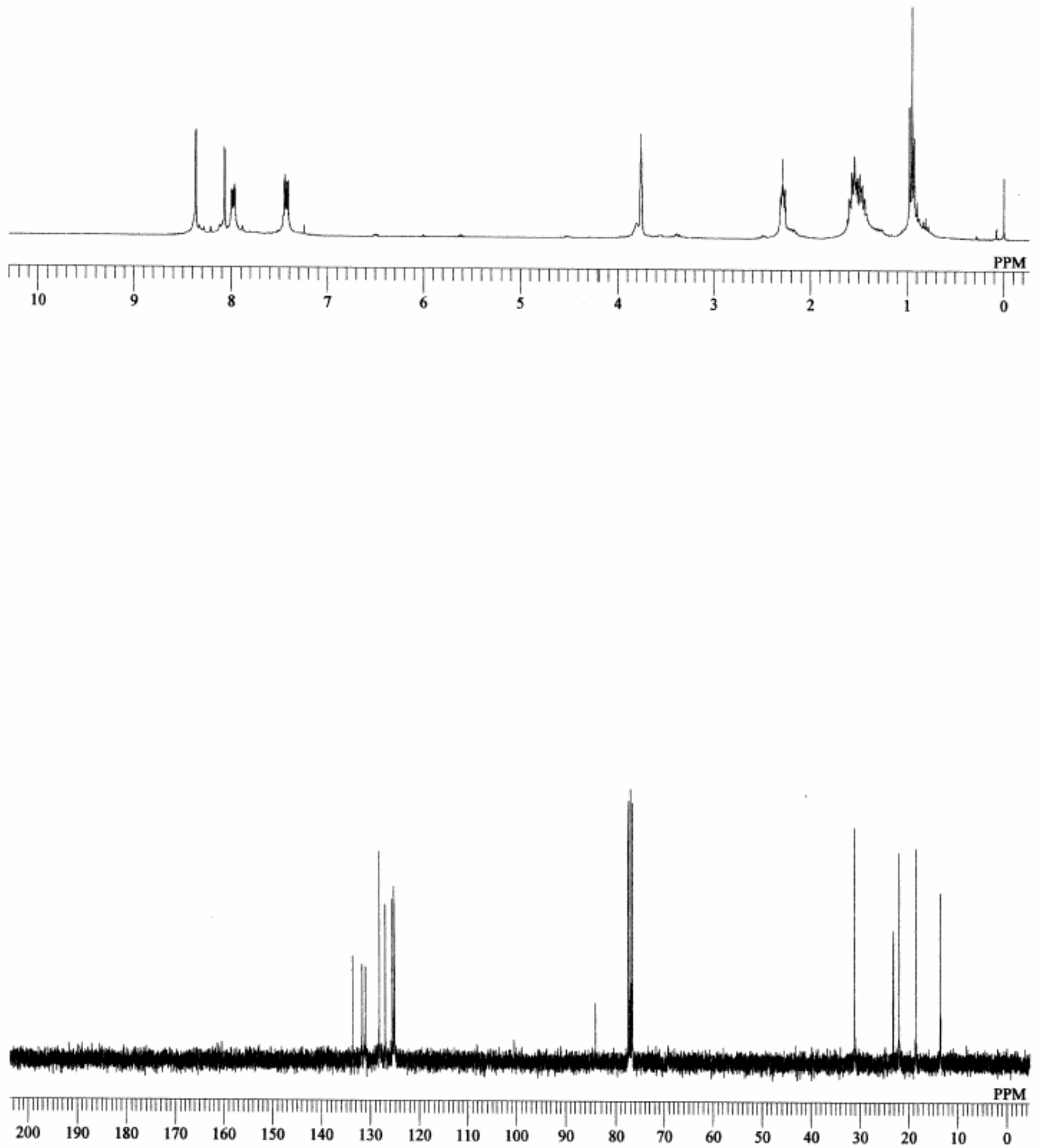
<smiles>CCc1c2c(c(CC)c(C(=O)OC)c1CC)Cc1c(c(CC)c3cc4c(CC(=O)OC)c(CC)c(CC)c(CC)c4cc3c1CC)C2</smiles>

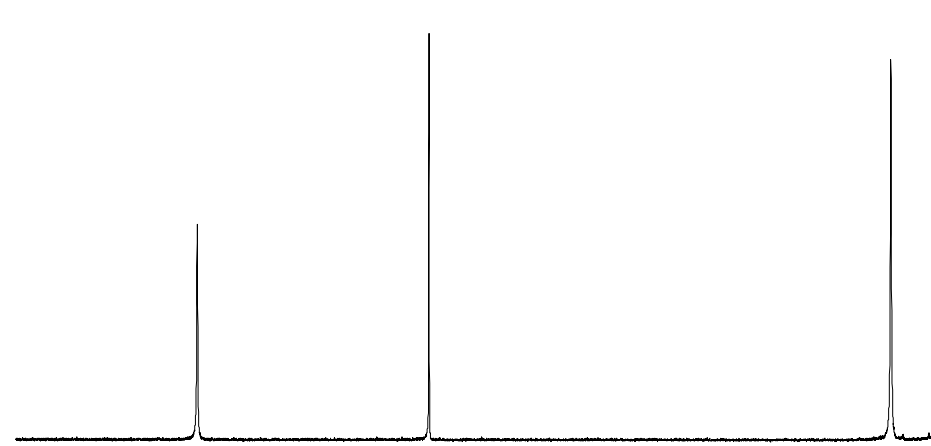

$8 a$ 
<smiles></smiles>

$8 b$

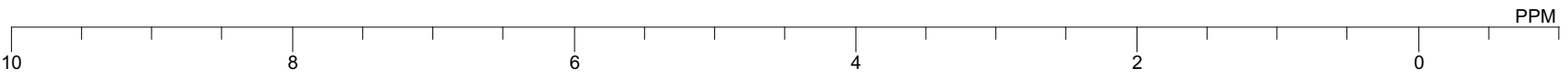


<smiles>CCc1c(CC)c(CC(C)(C)C)c2cc3c(Br)c4c(c(Br)c3cc2c1CC)Cc1c(Br)c(C(=O)OC)c(C(=O)OC)c(CBr)c1C4</smiles>

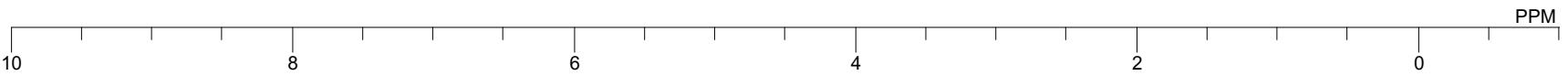

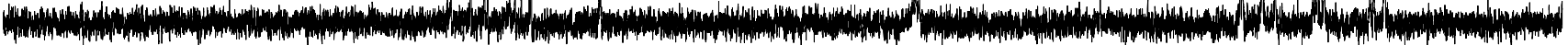

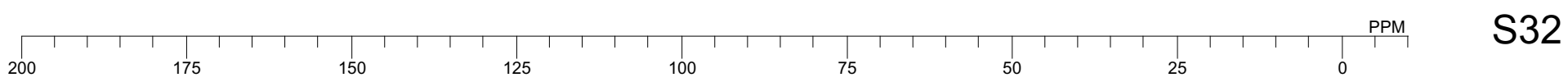




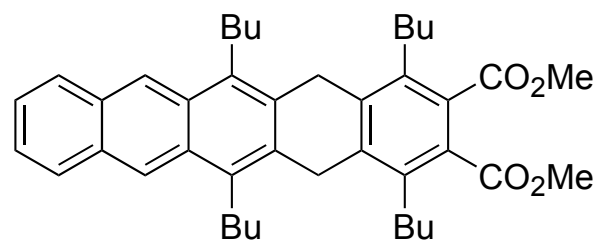

8d
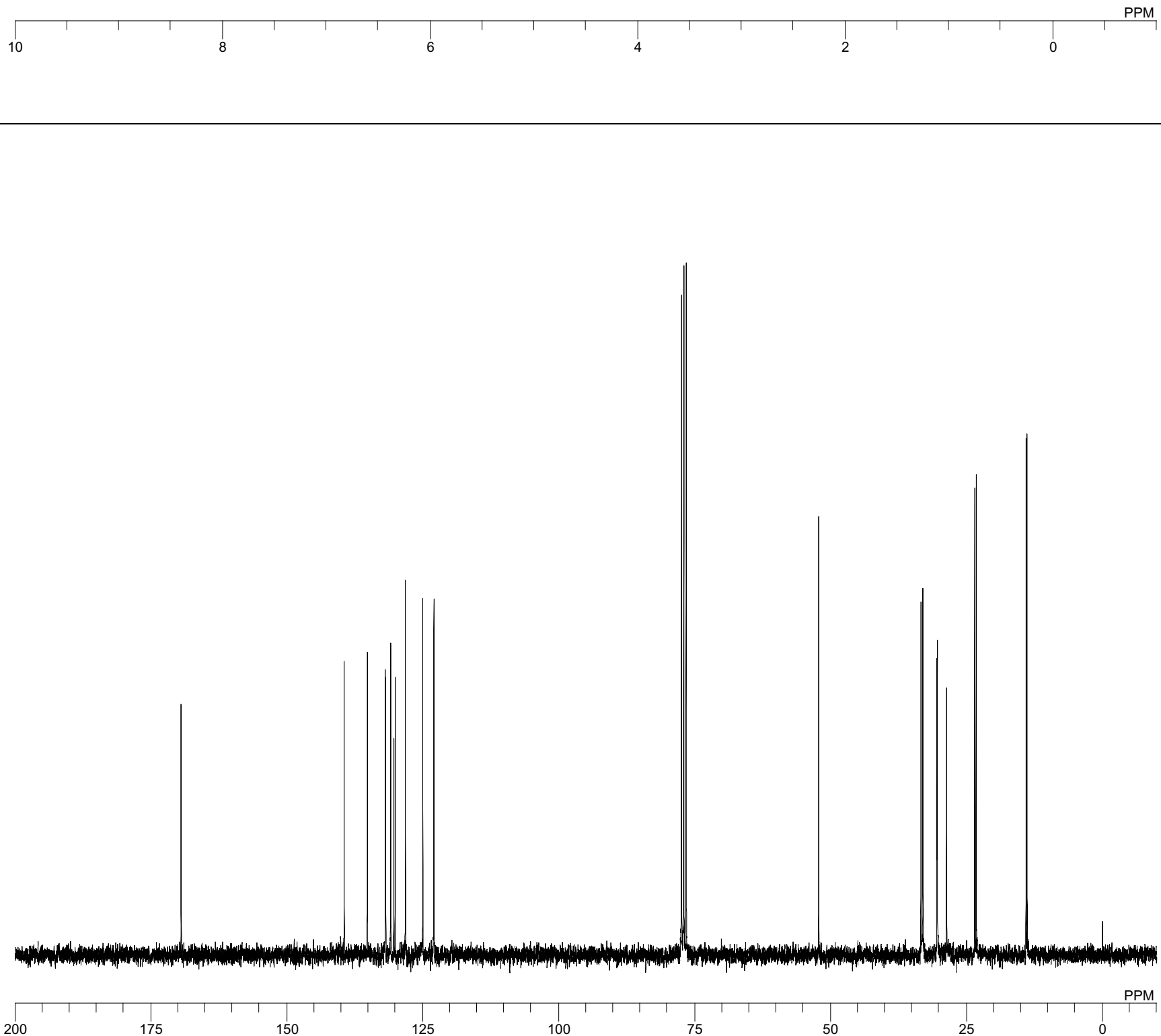


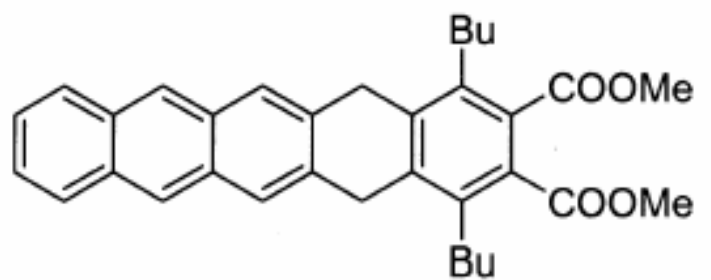

1,4-Dibutyl-5,14-dihydro-pentacene-2,3dicarboxylic acid dimethyl ester
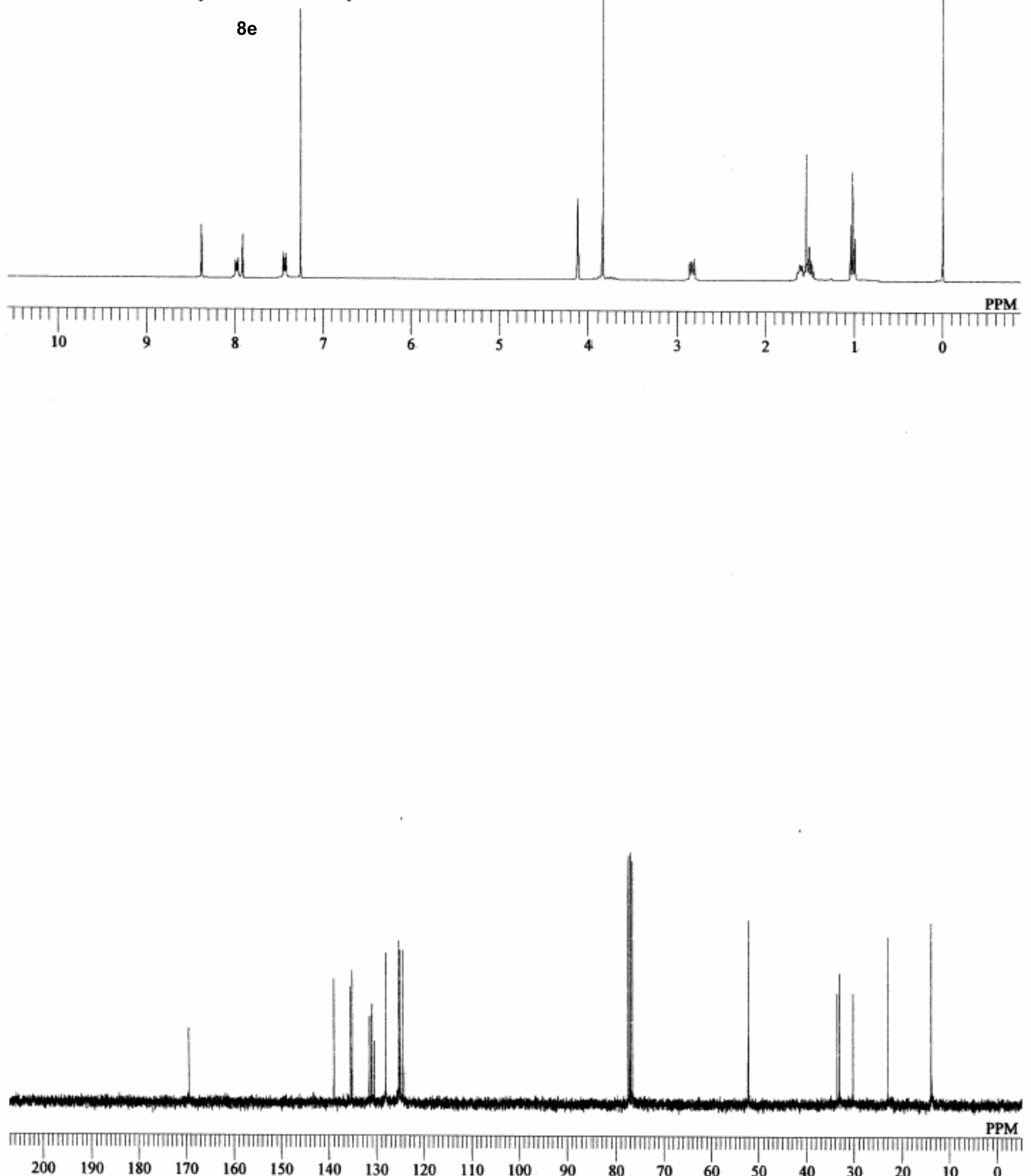
<smiles>CCc1c(CC)c(CC)c2cc3c(cc2c1CC)c1cc2c(CC)c(C(=O)OC)c(CC)c(CC)c2cc1c1cc2c(C(=O)OC)c(CC)c(CC)c(CC)c2cc31</smiles>

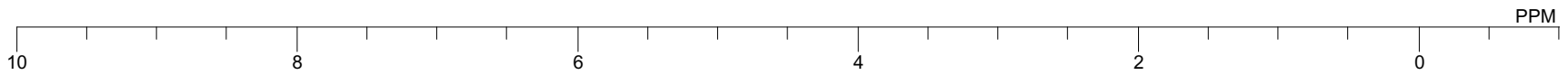


<smiles>CCCCc1c(C(=O)OC)c(C(C)=O)c(CCC)c2cc3c(CCC)c4cc5c(CC)c(CC)c(CC)c(CC)c5cc4c(CCC)c3cc12</smiles>
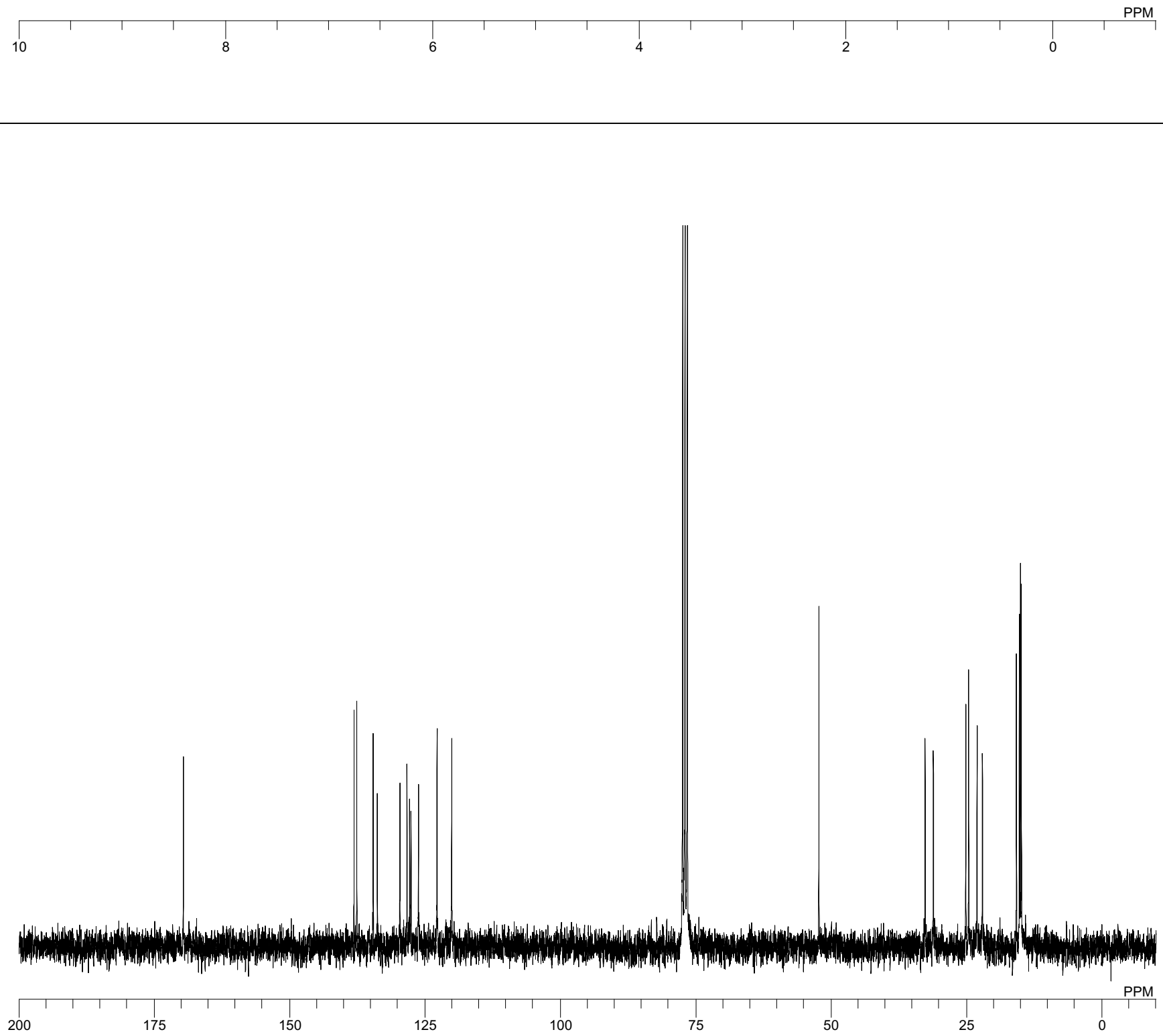
<smiles>CCc1c(CC)c(CC)c2cc3c(C(=O)c4ccccc4)c4cc5c(C(=O)OC)c(C(=O)OC)c(CC)c(CC)c5cc4c(C(=O)c4ccccc4)c3cc2c1CC</smiles>

9c

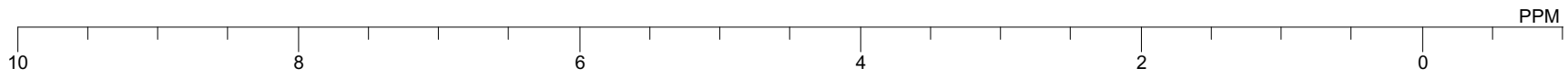

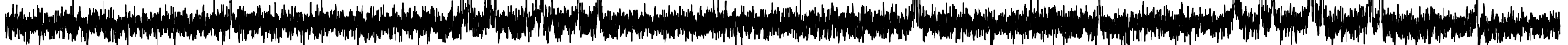

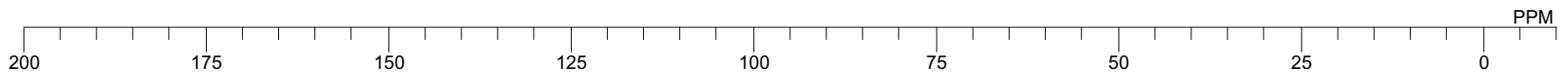




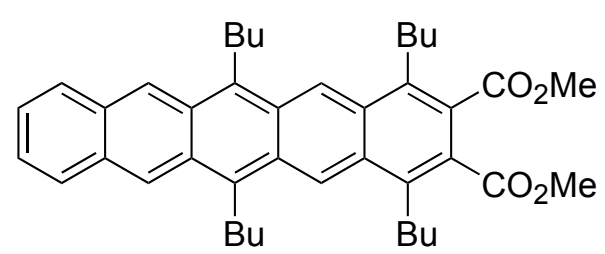

9d

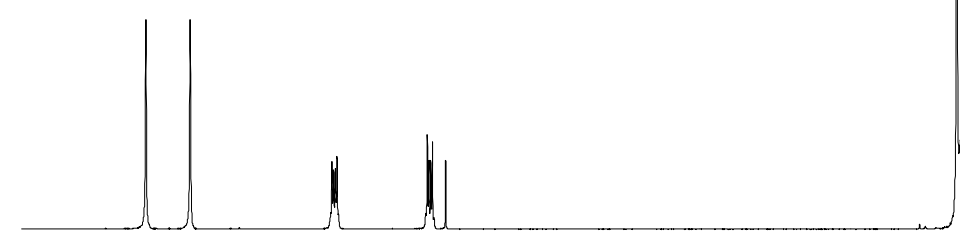
PPM 


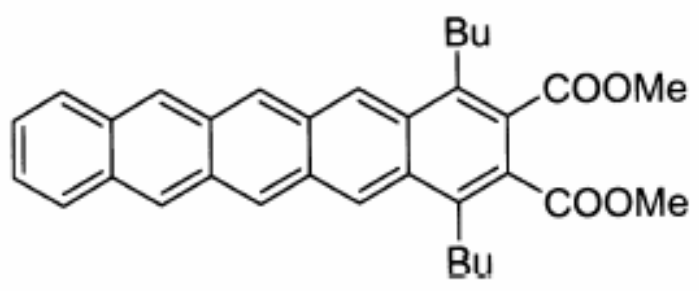

1,4-Dibutyl-pentacene-2,3-dicarboxylic acid dimethyl ester

$9 e$
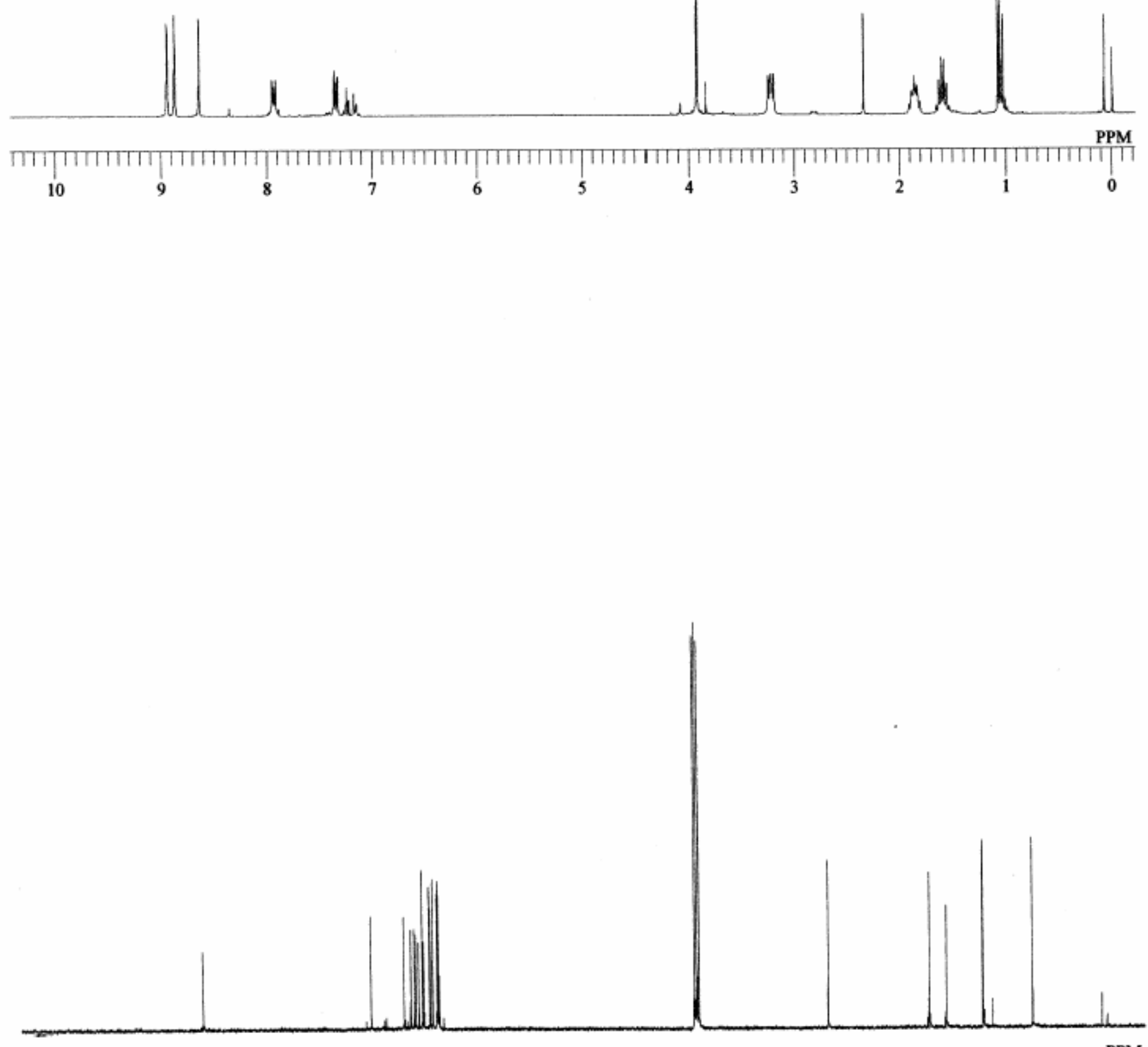

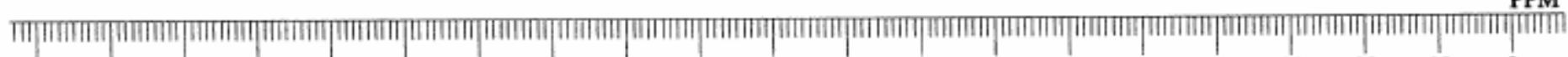

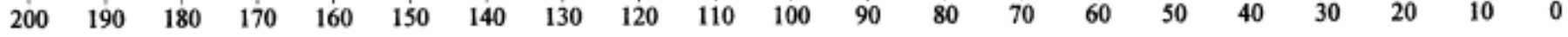



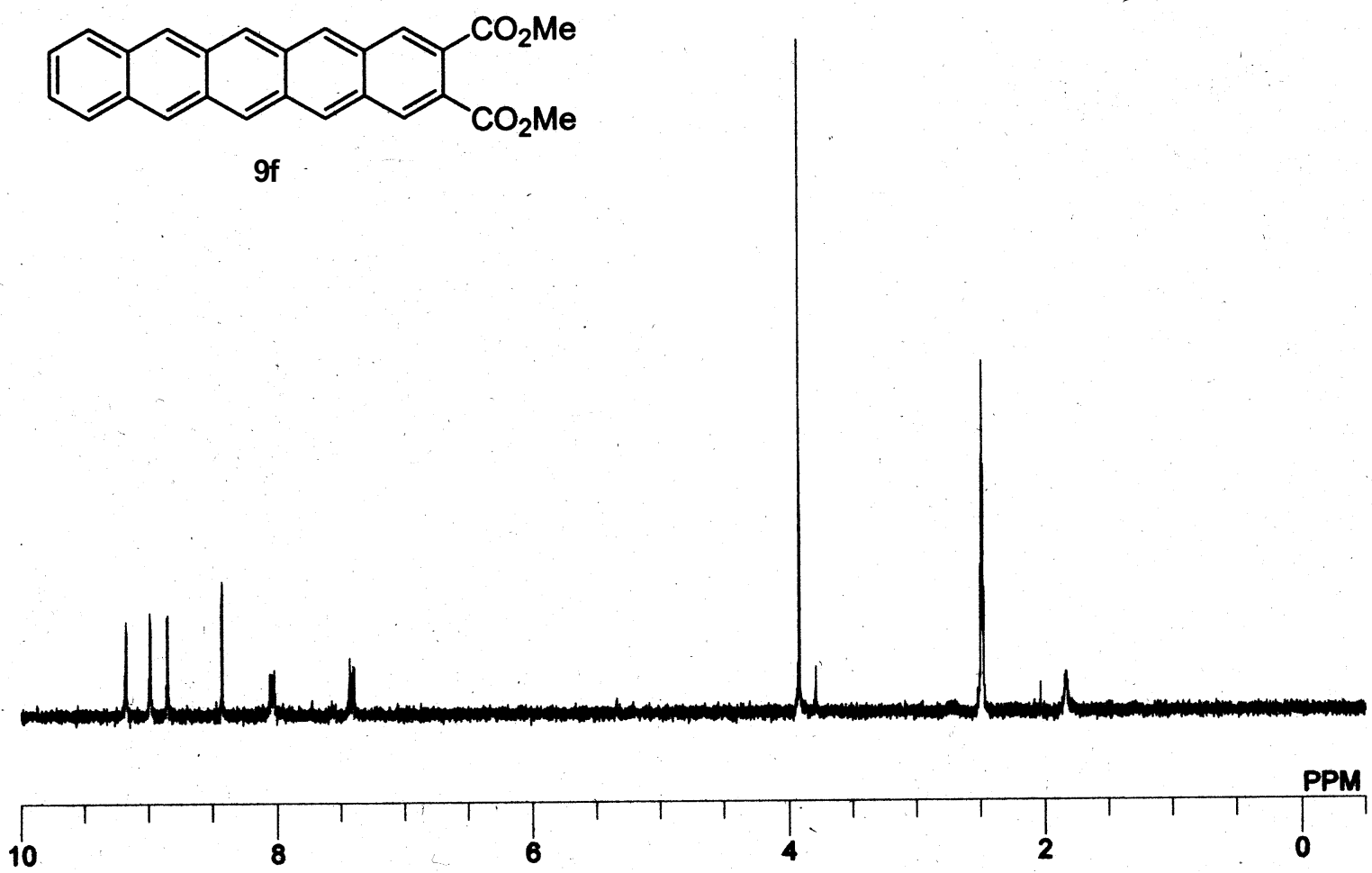


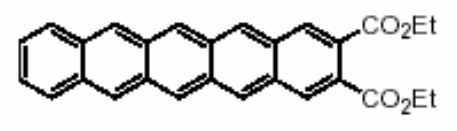

$9 \mathrm{~g}$

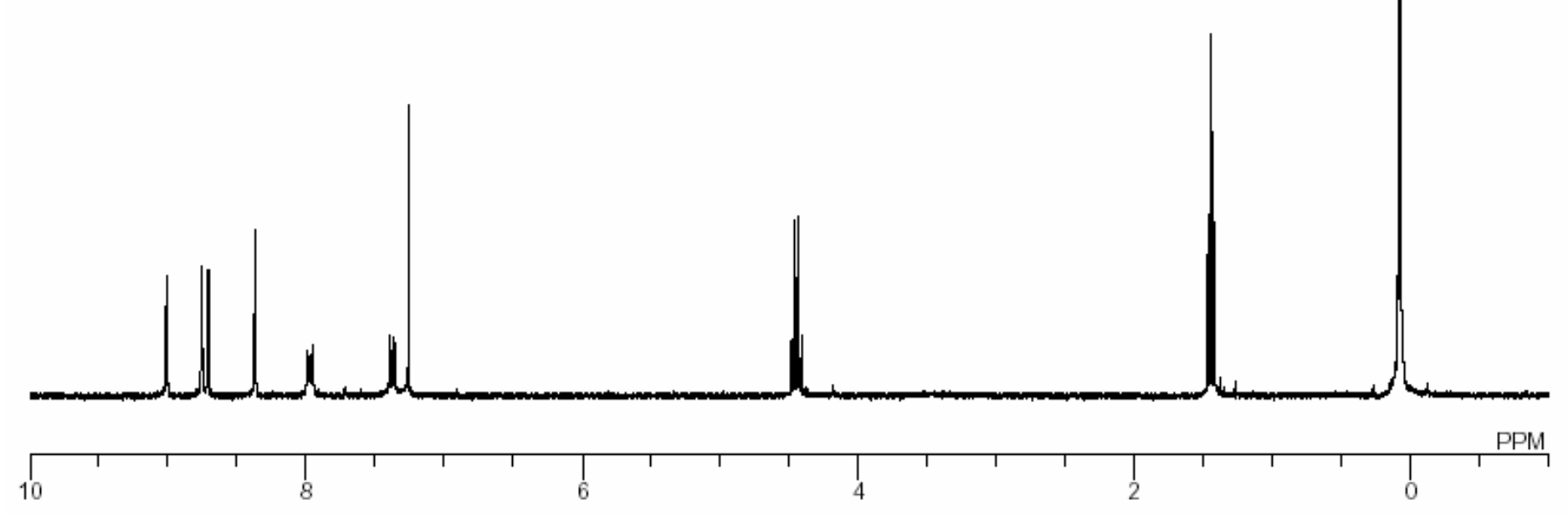

IIIIII

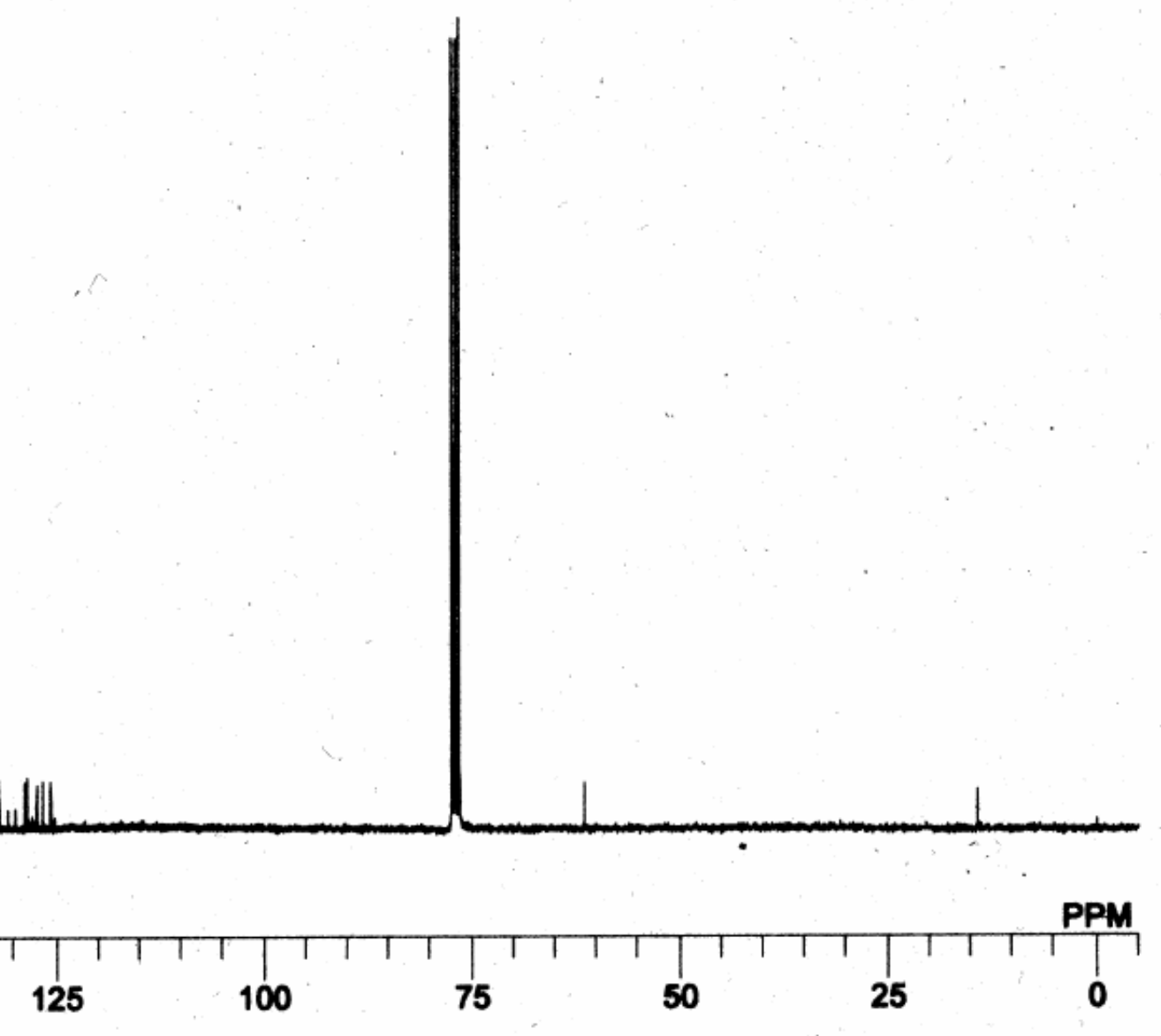




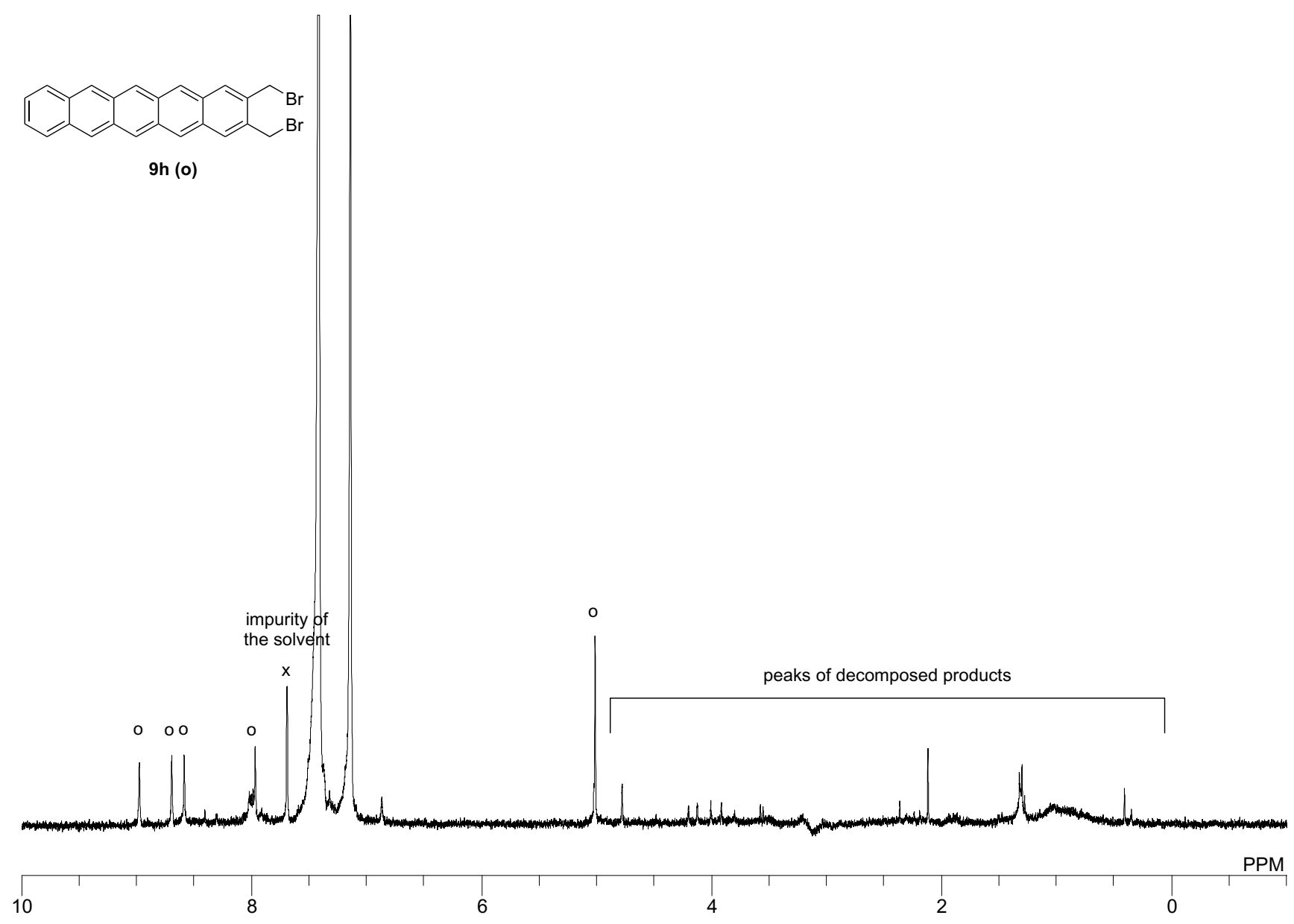

S42 
nitrobenzene

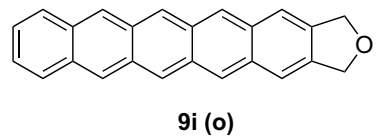

9i (o)

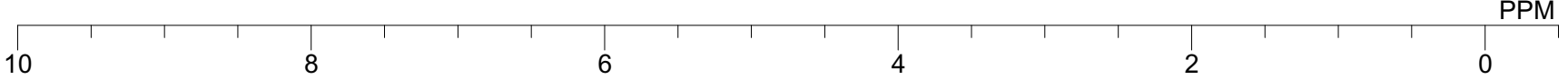


<smiles>OCc1cc2c(cc1CO)Cc1ccccc1C2</smiles>

4g

ঙ্ల

$\stackrel{n}{=}$

1
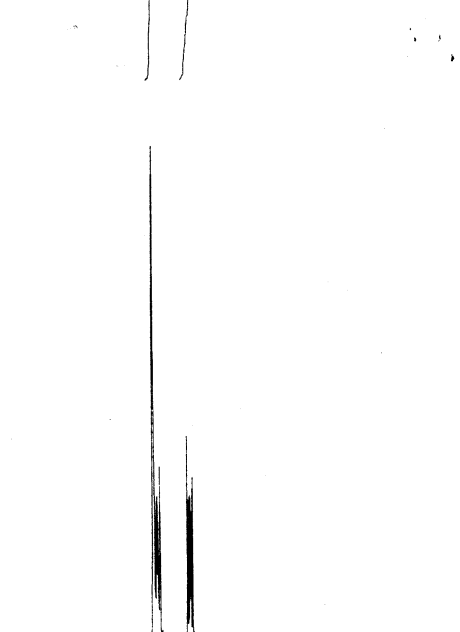

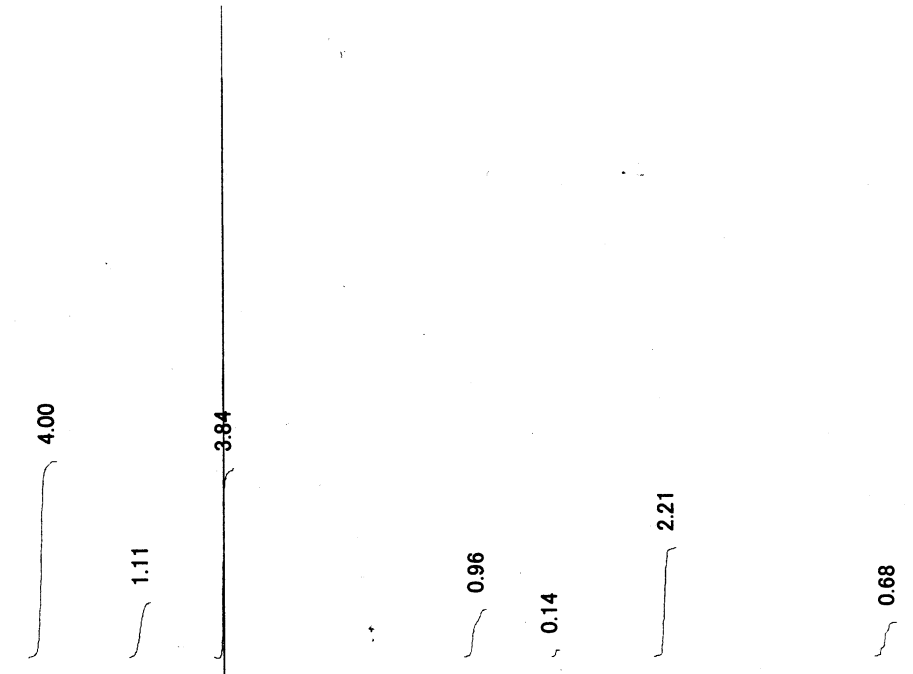

:

(1) 

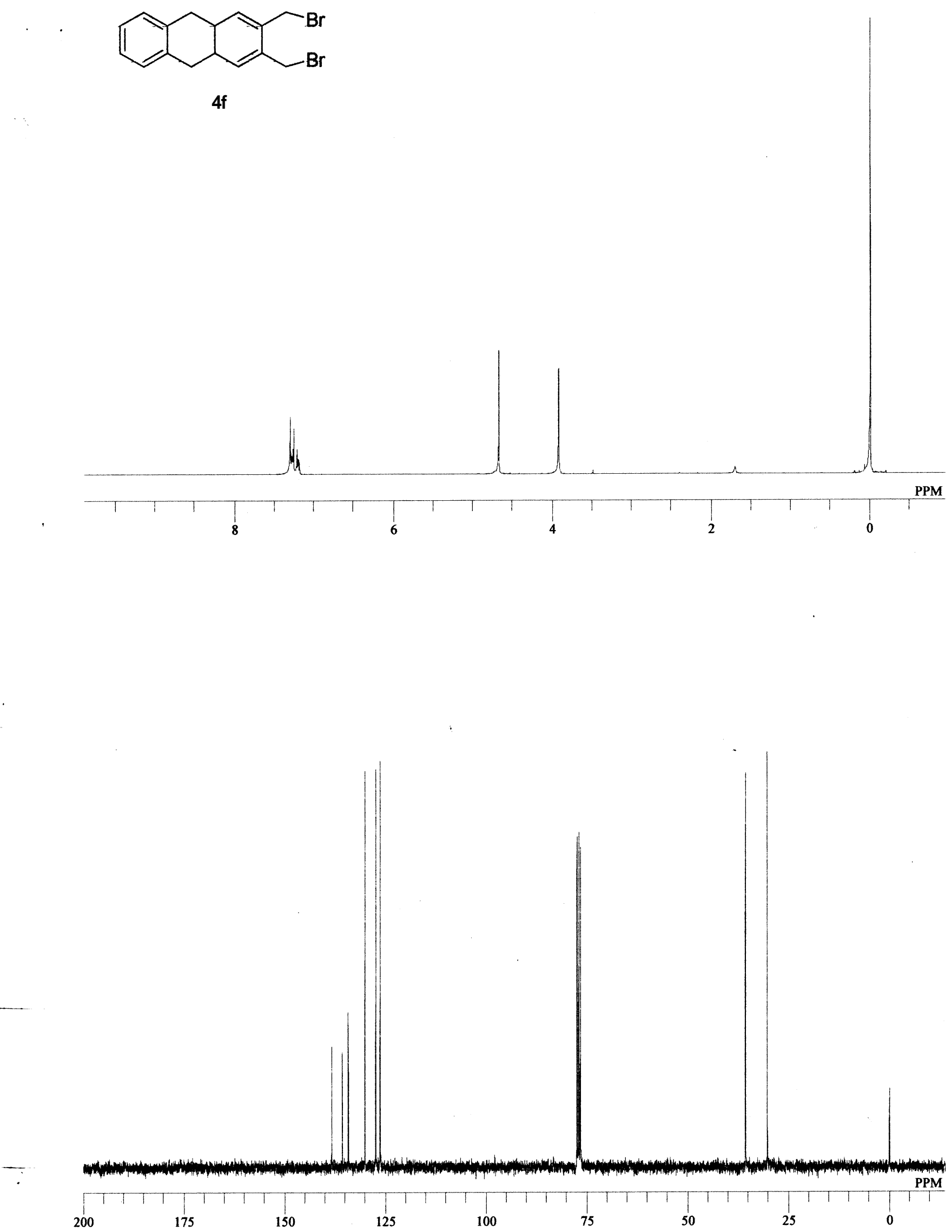

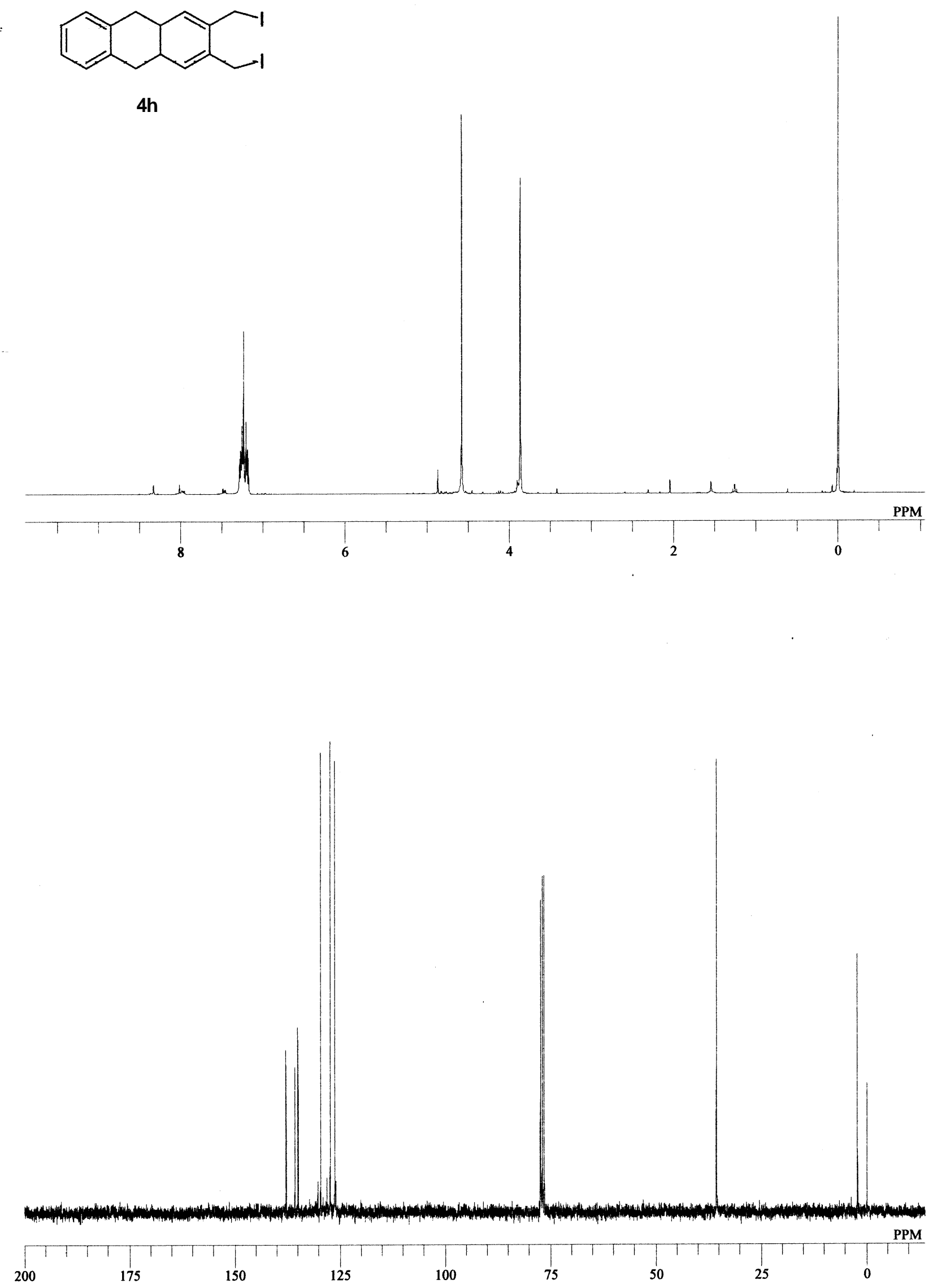


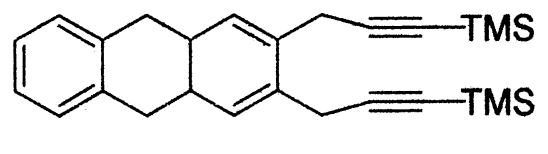

10
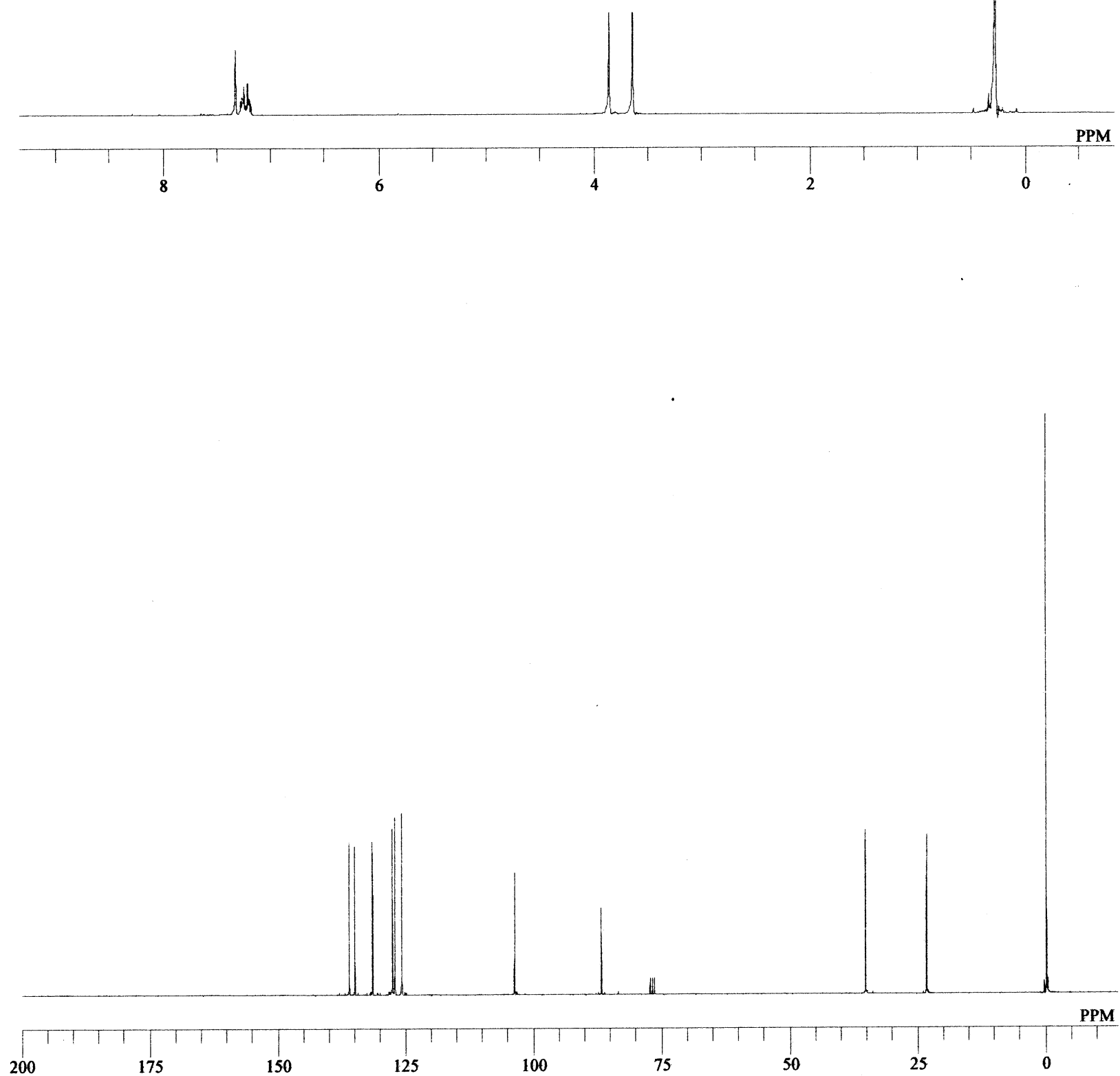


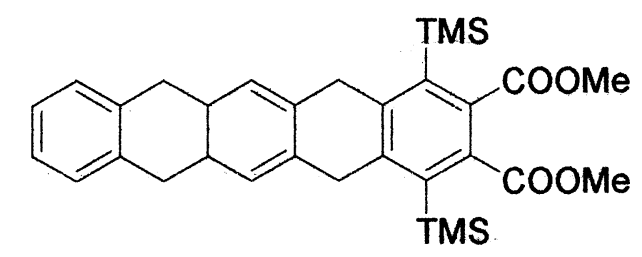

$11 a$
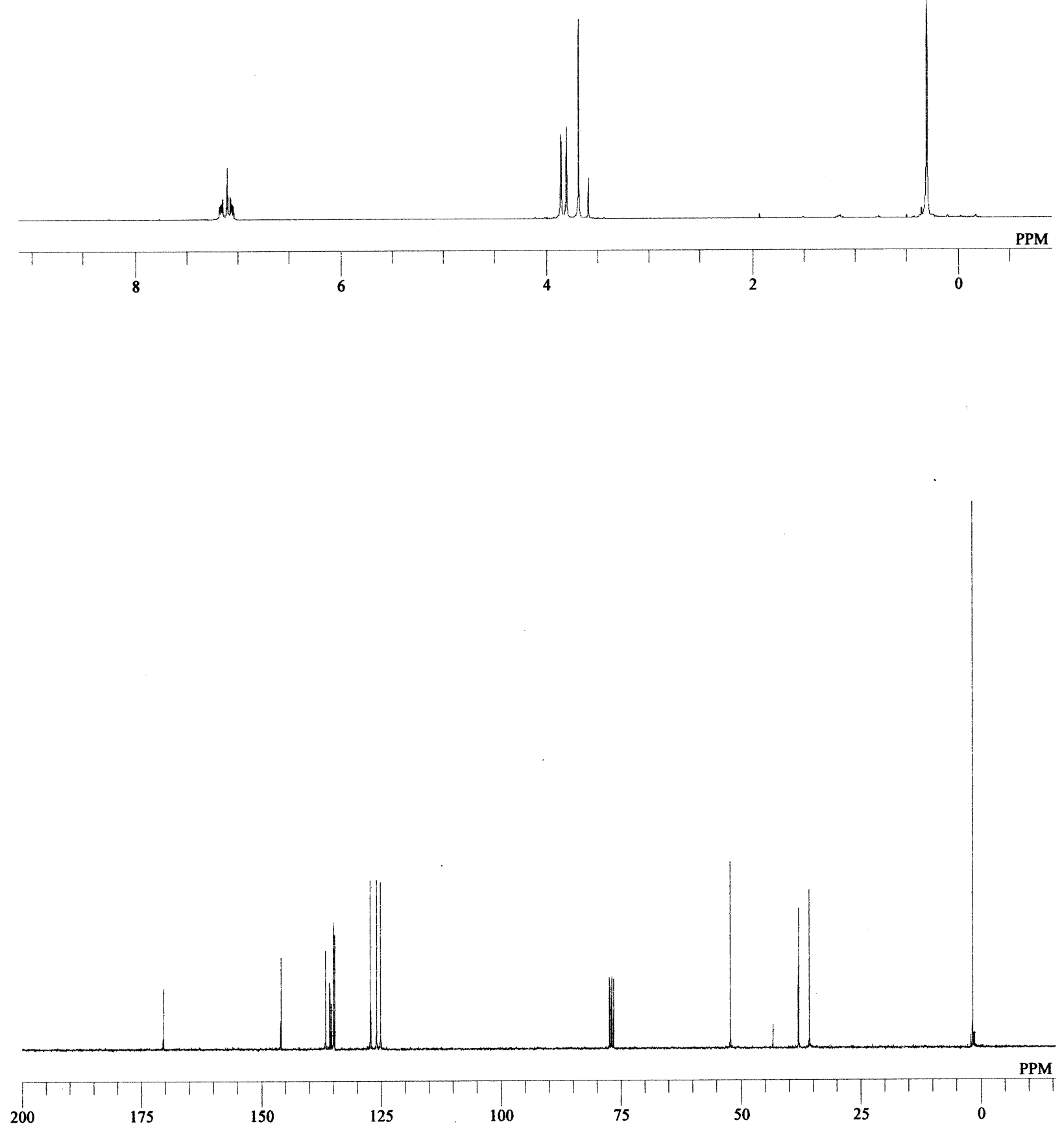


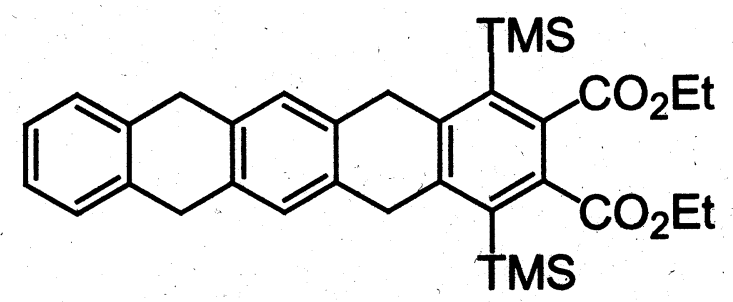

11b
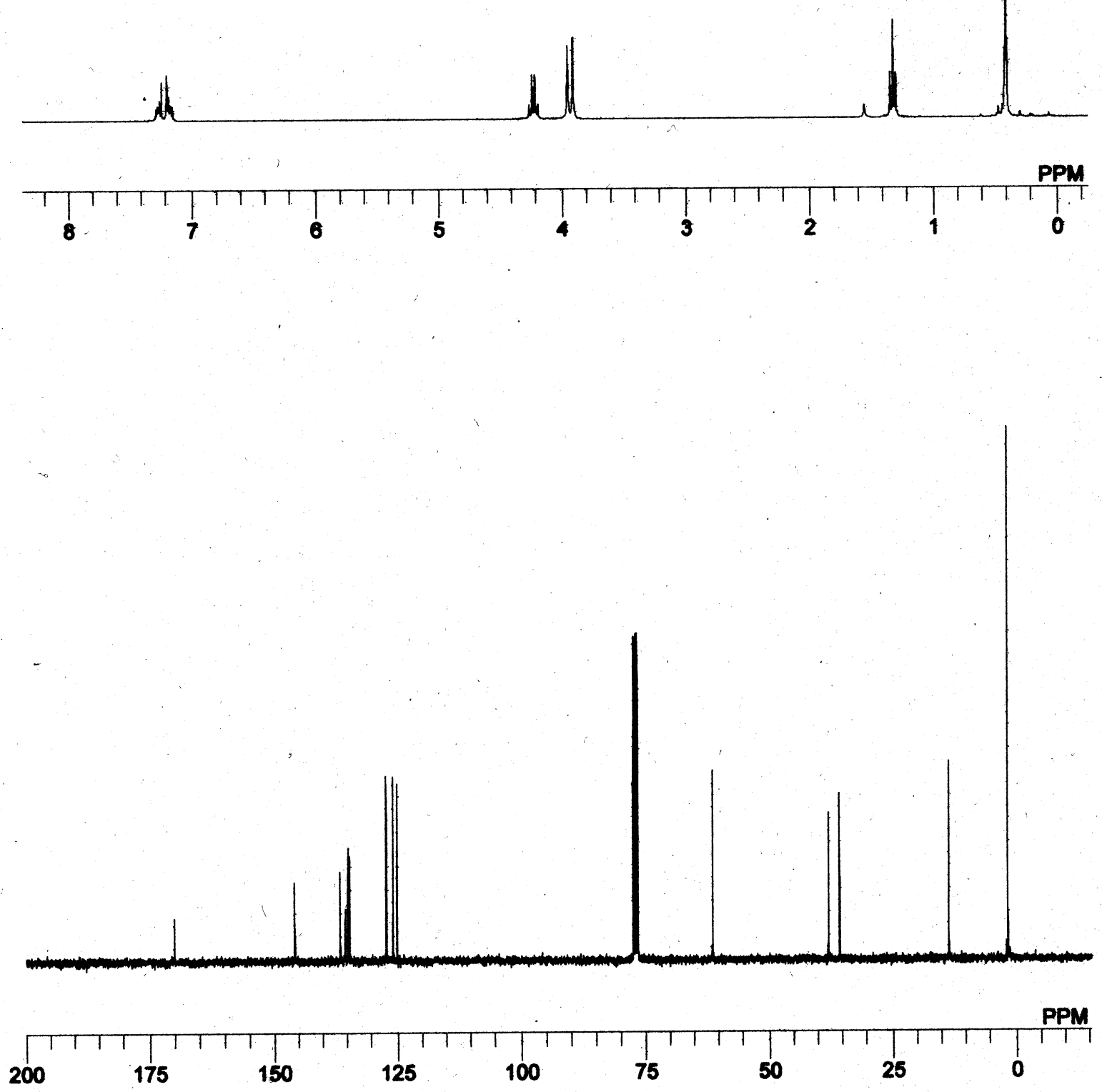

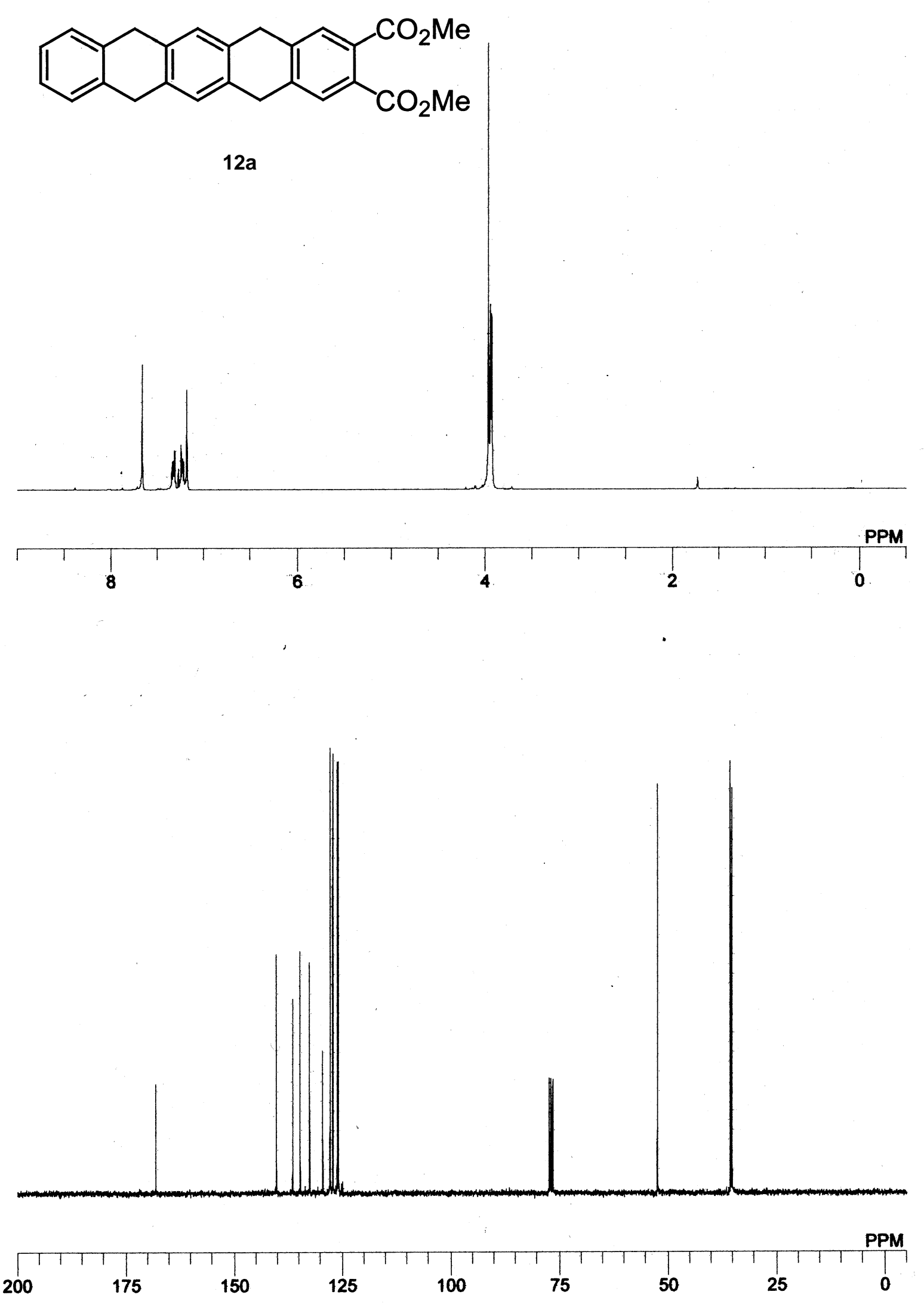


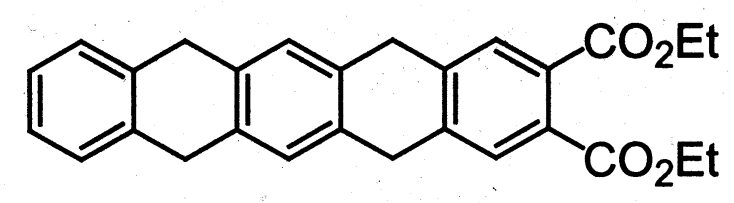

$12 b$
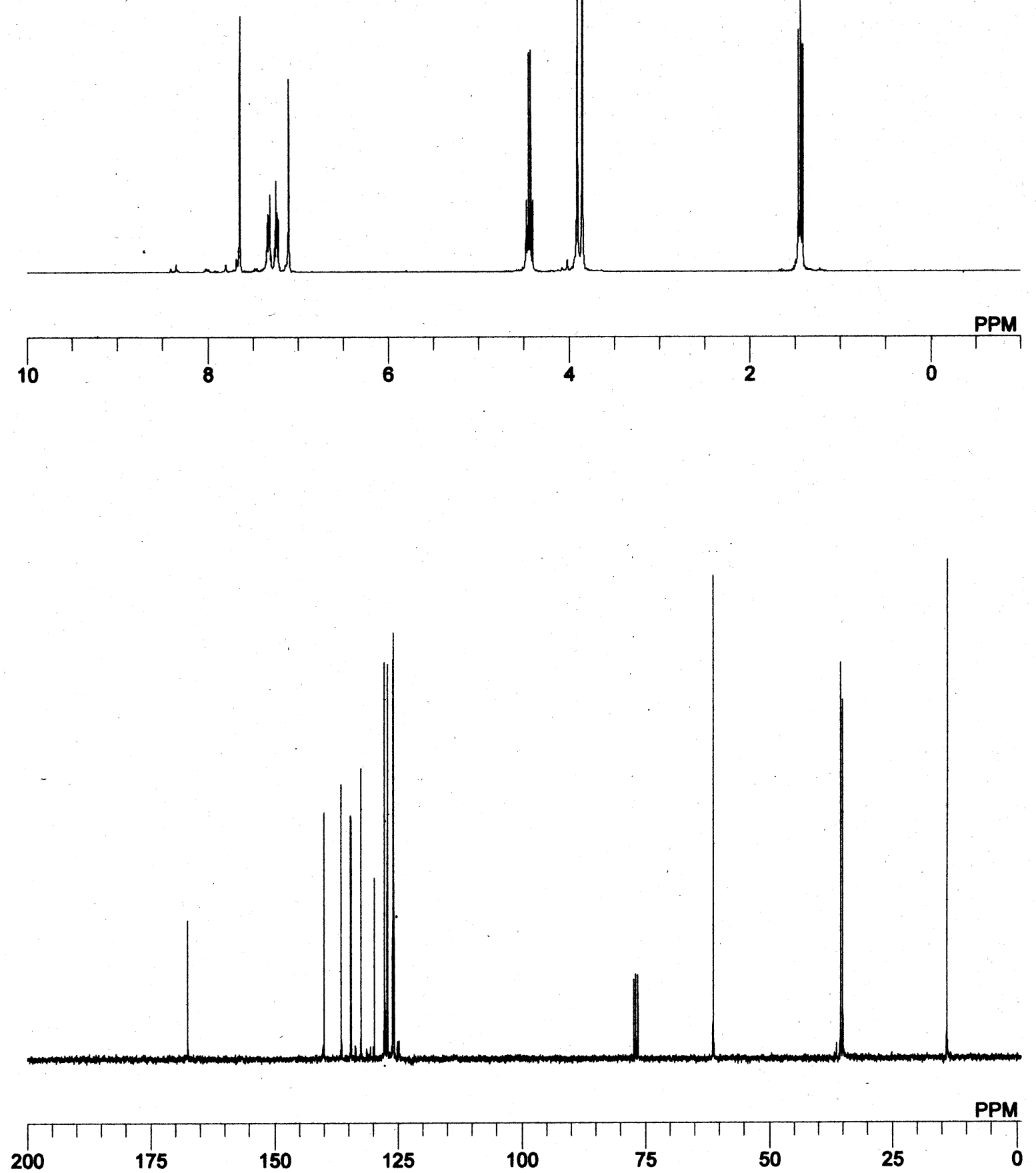

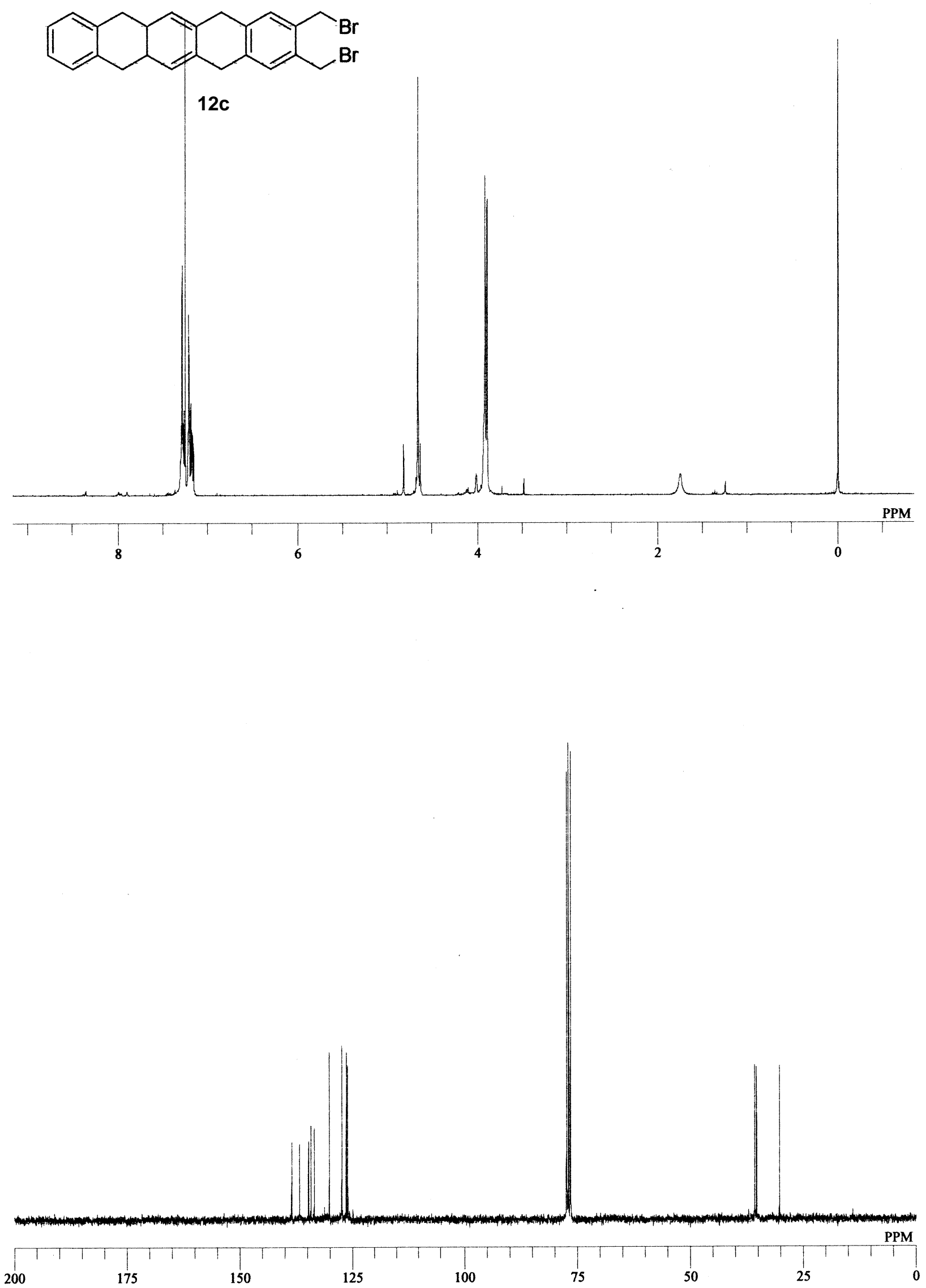

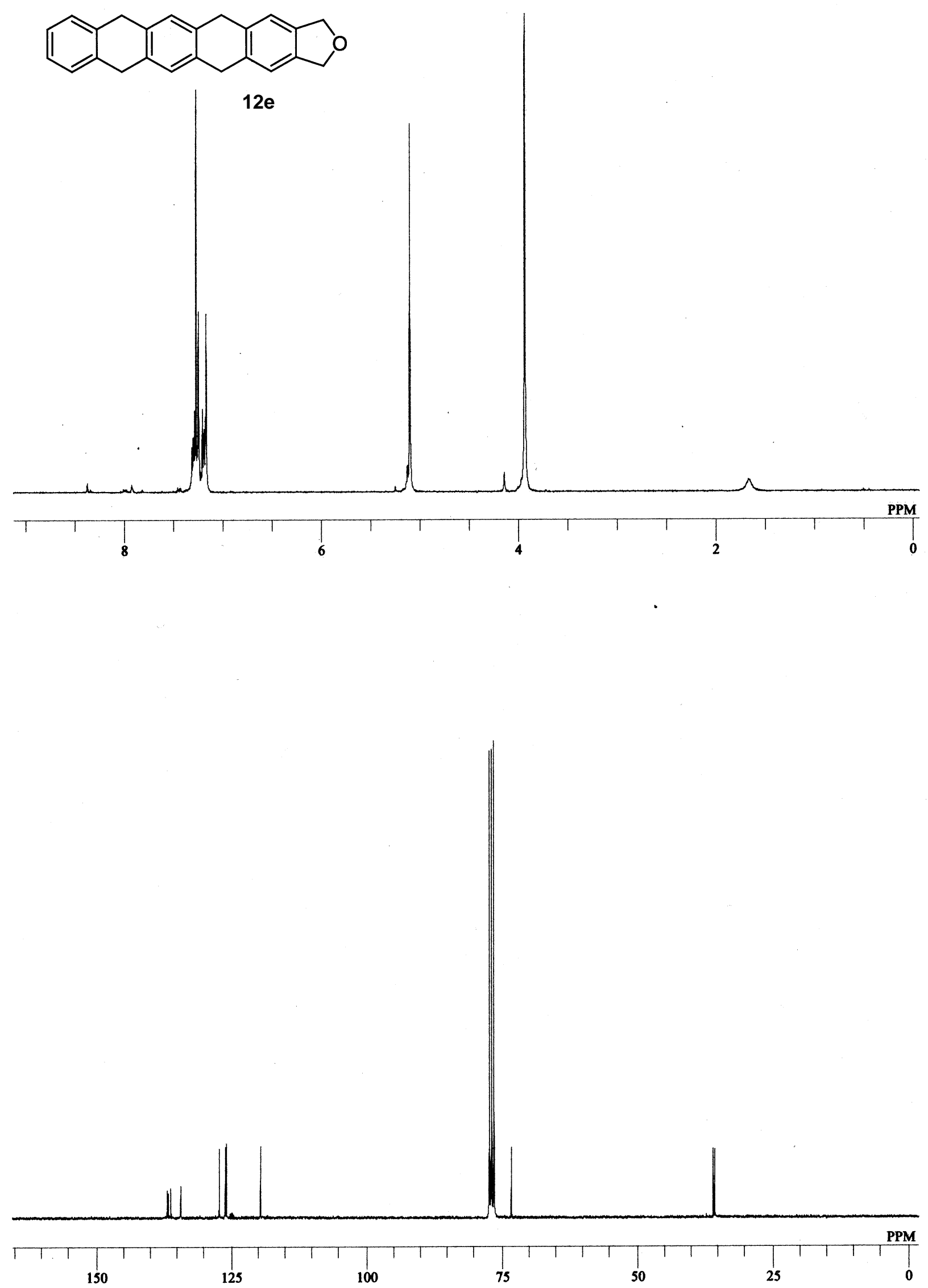
<smiles>Cc1c2c(c(C(C)C)c3c1Cc1cc4c(cc1C3)Cc1ccccc1C4)COC2</smiles>

12d
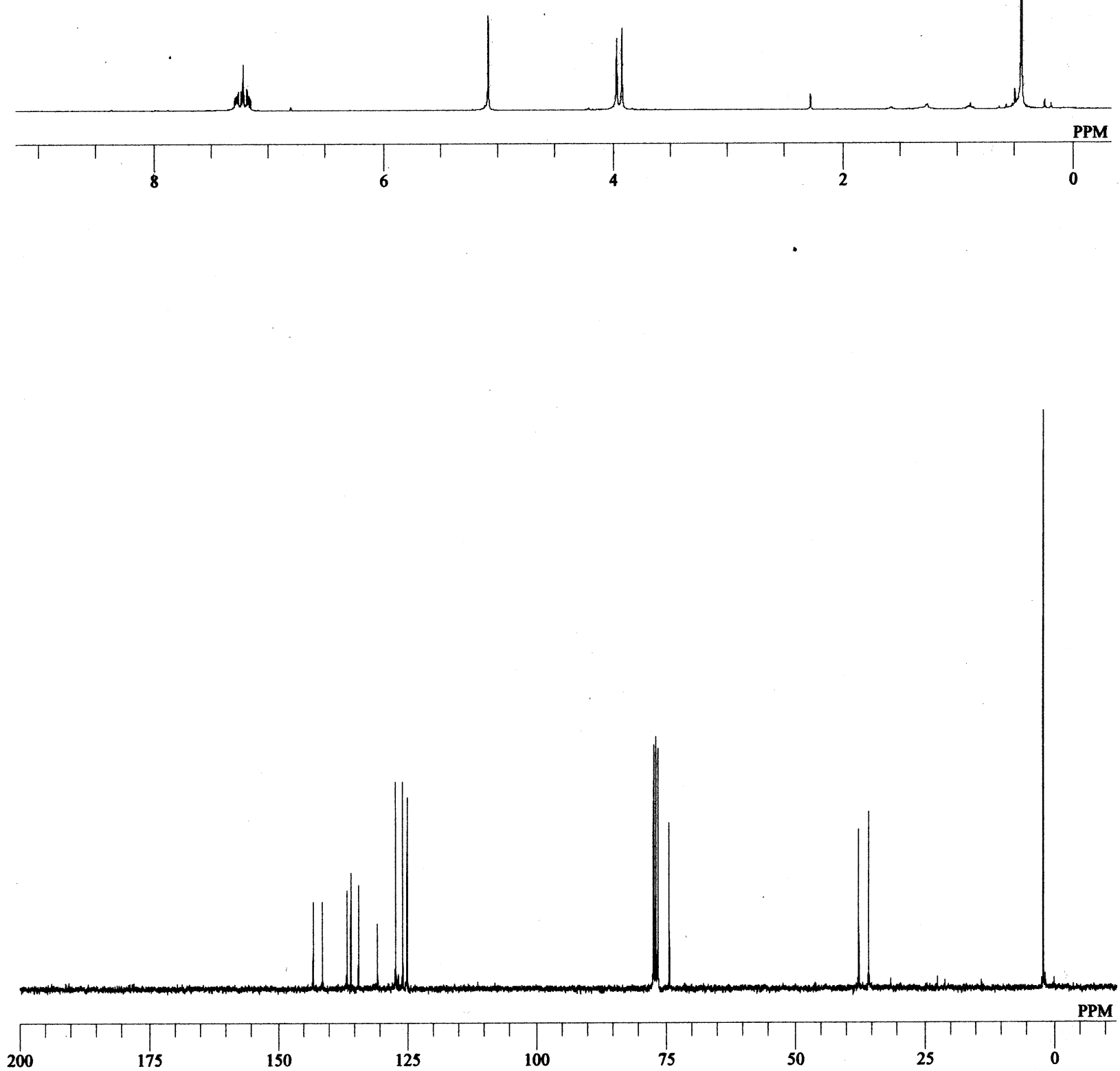


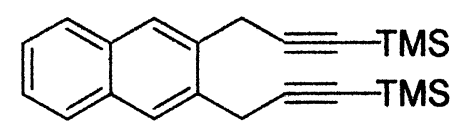

14
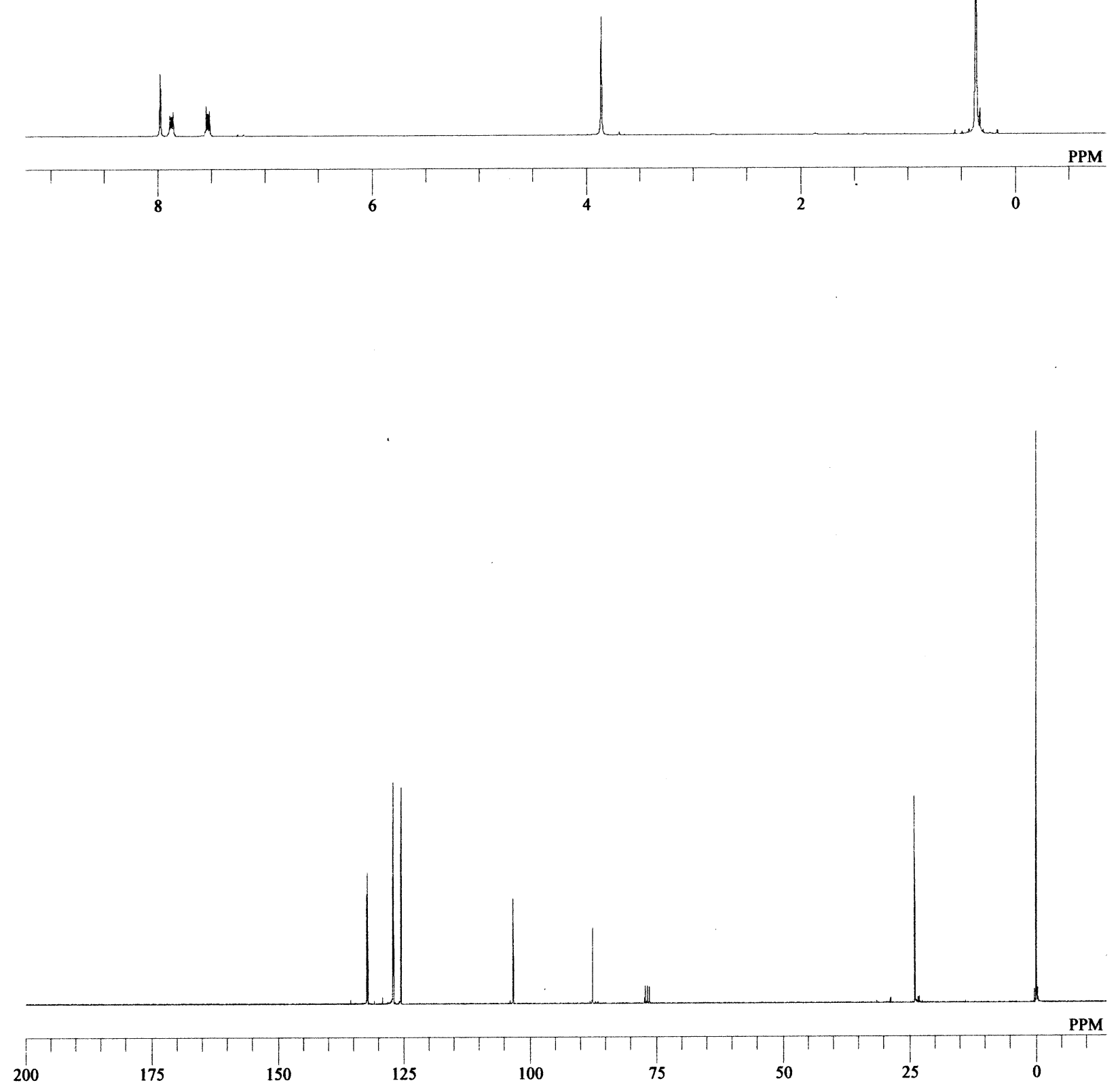


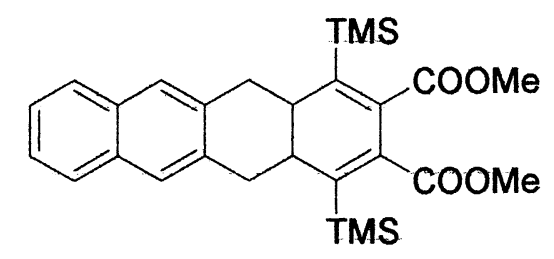

$15 a$
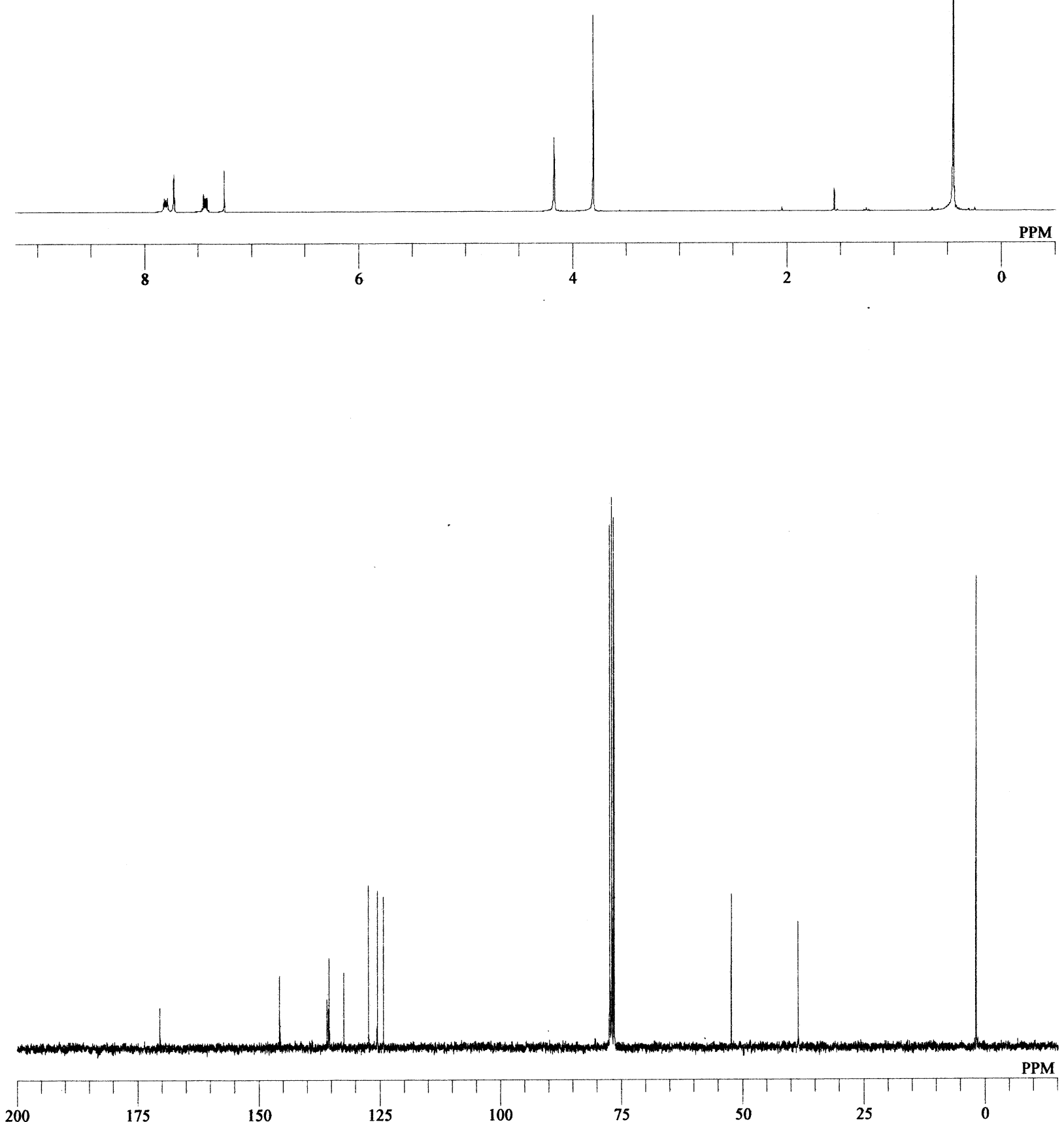

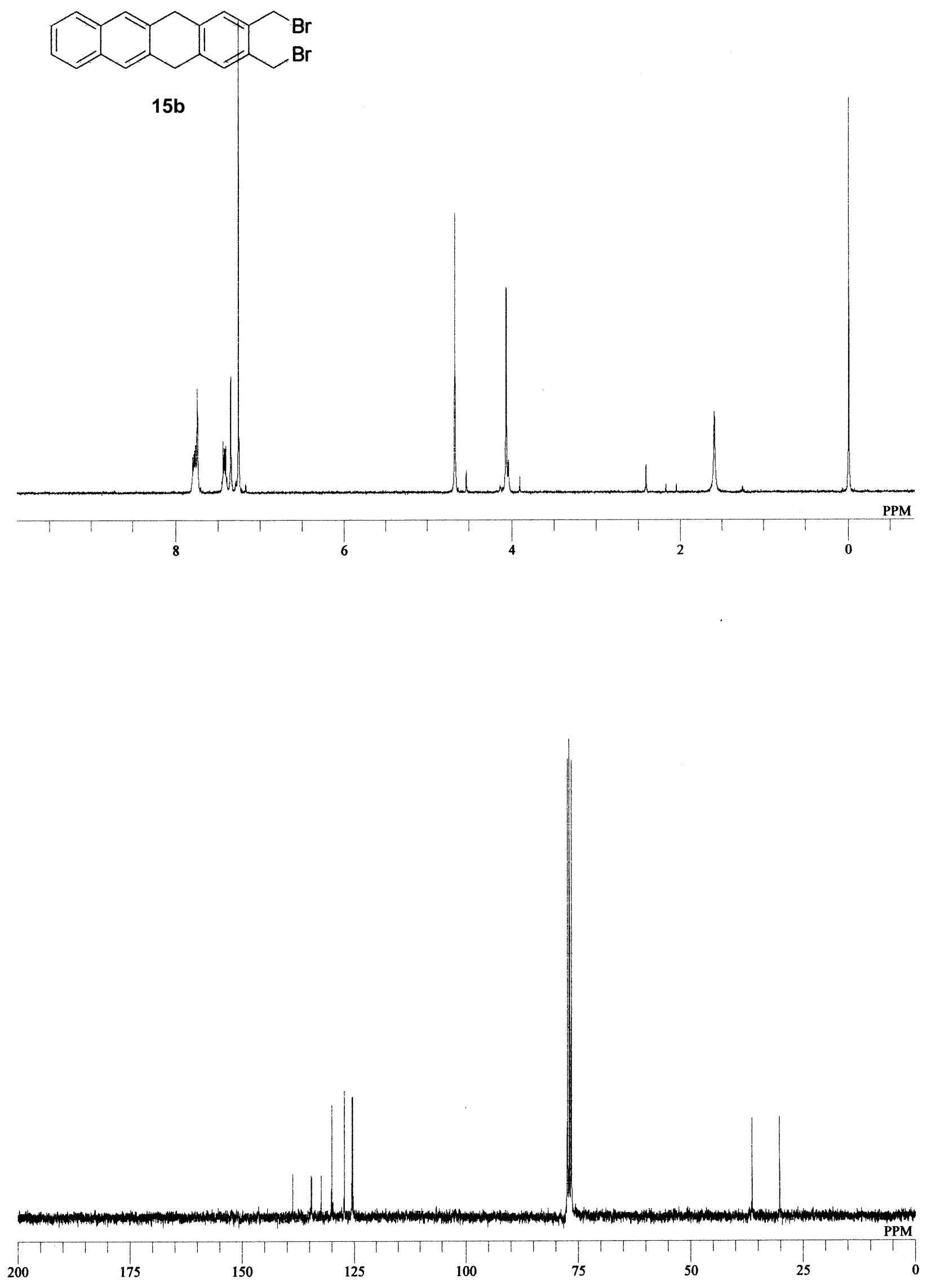


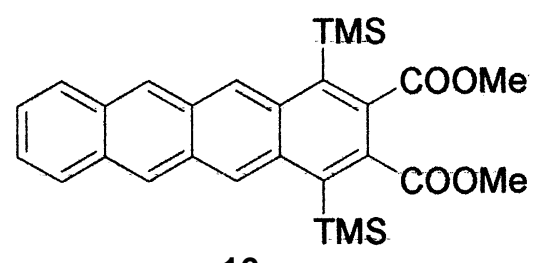

$16 a$
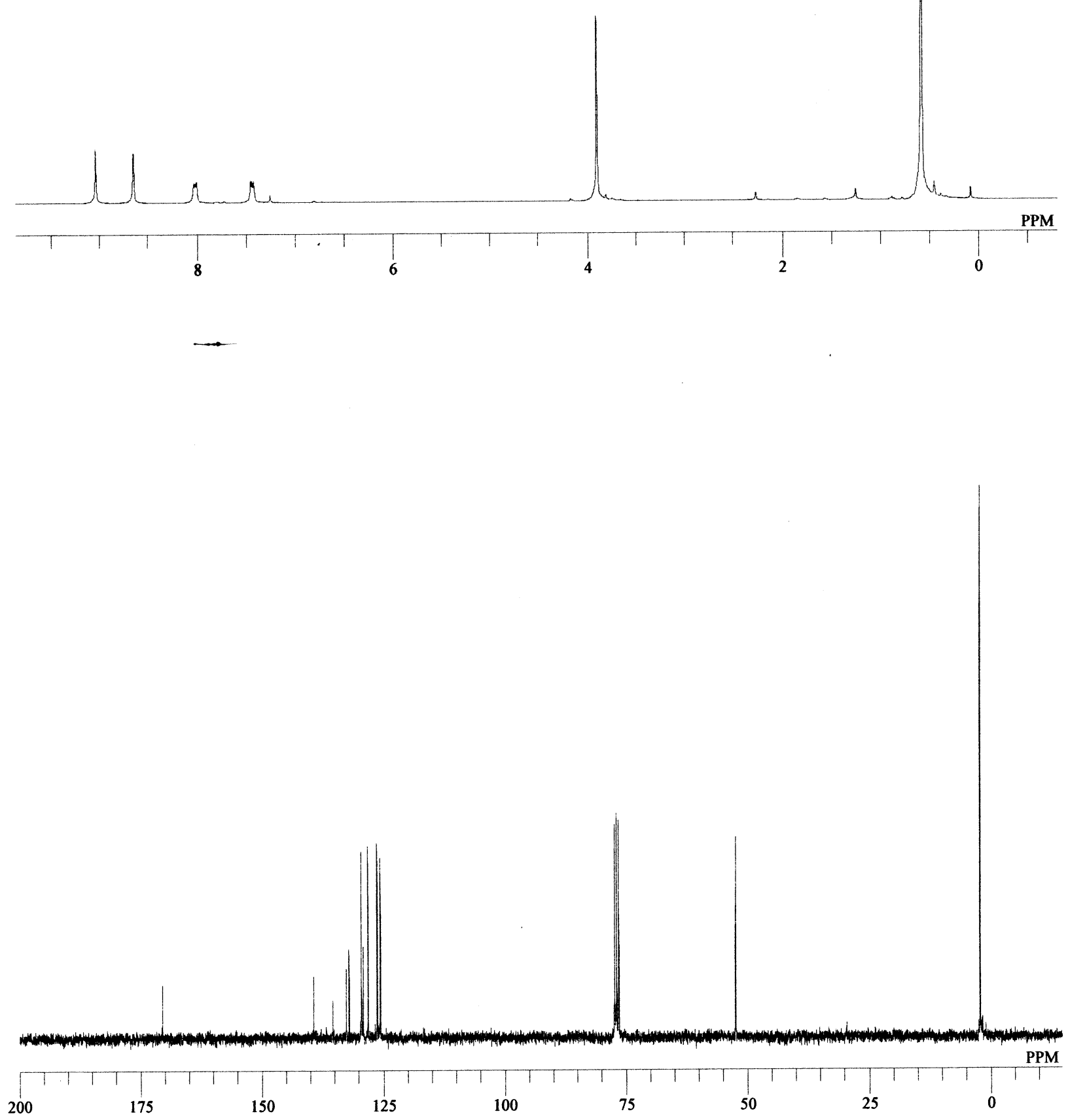

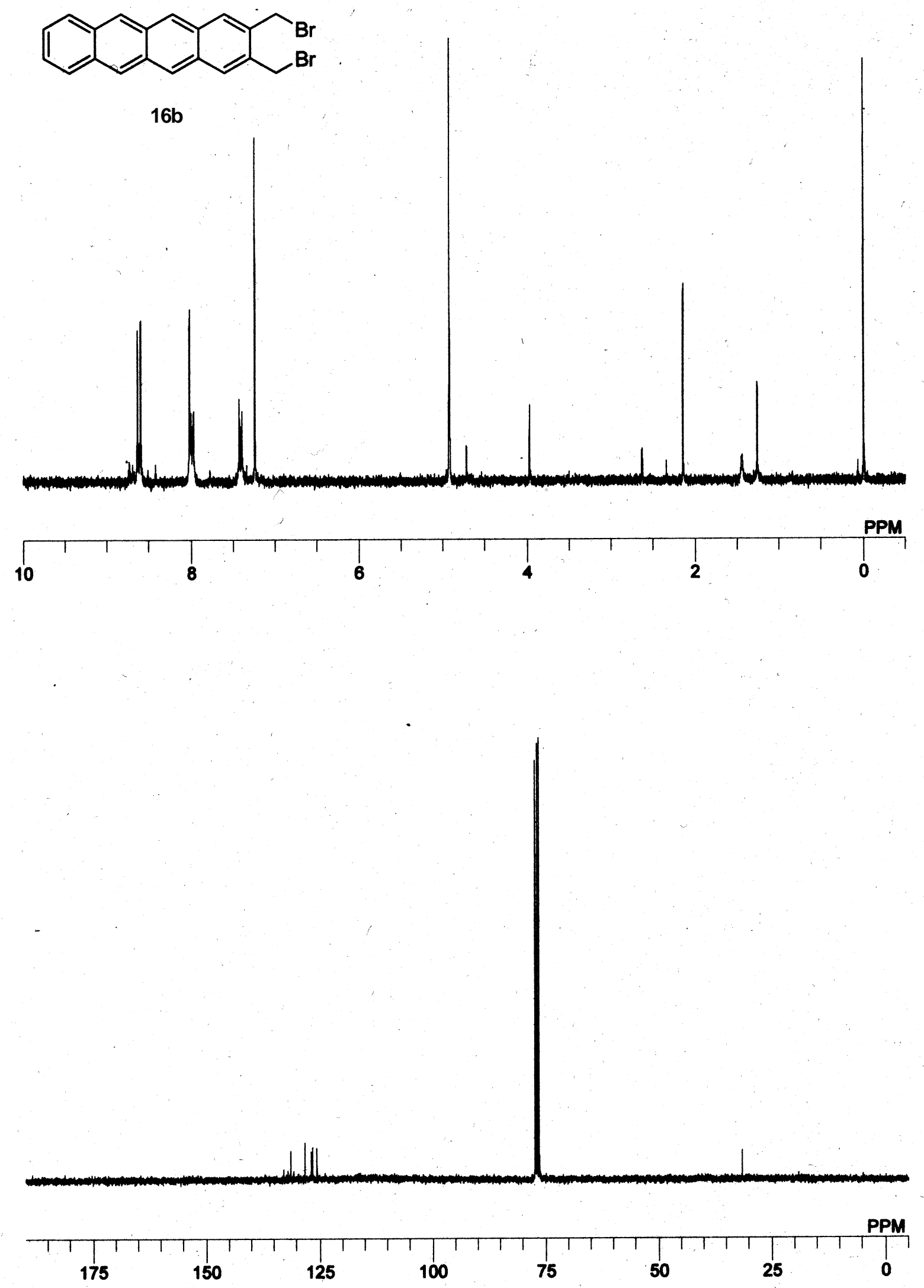


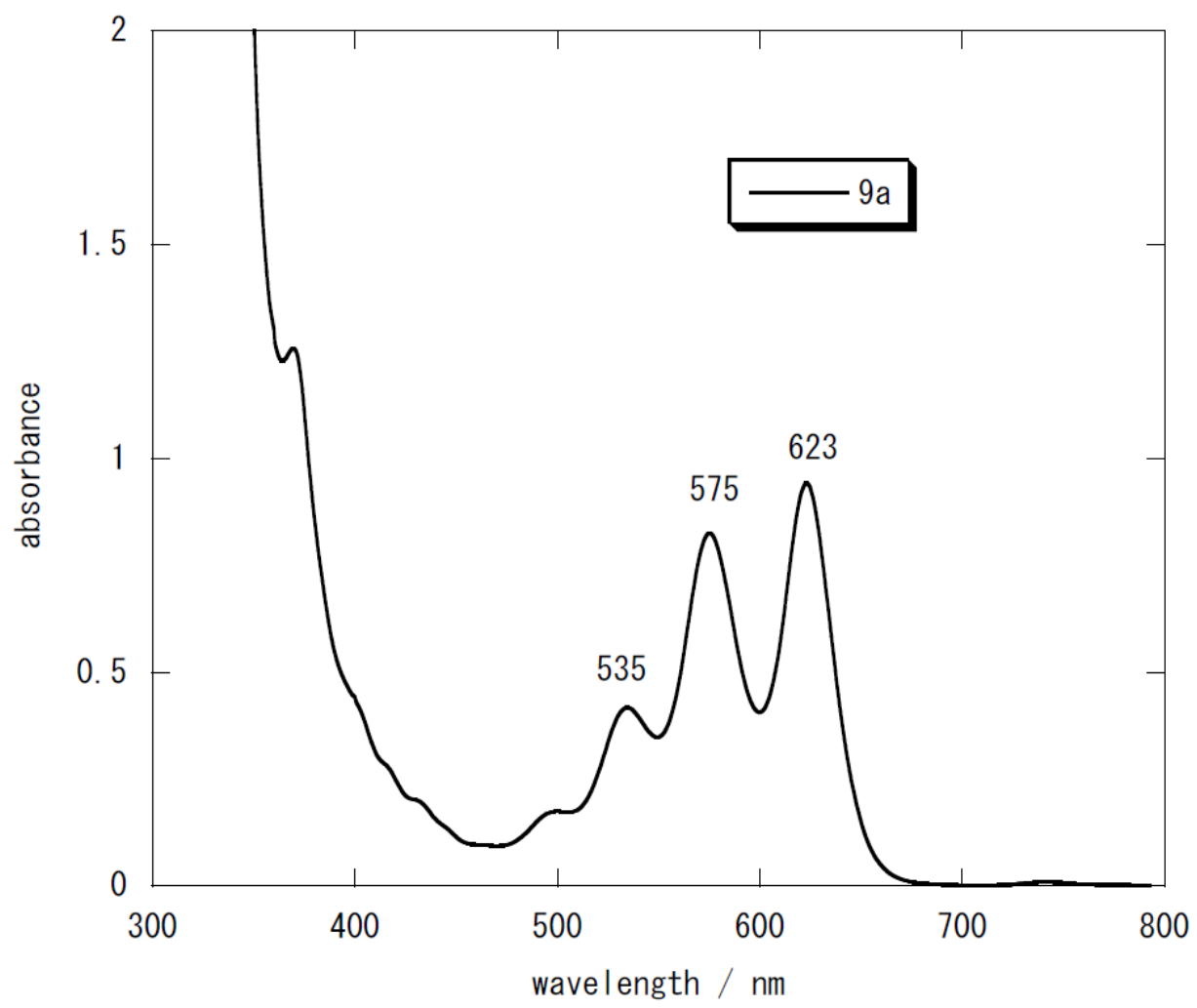

Figure 1. Absorption spectrum of pentacene 9a in THF at rt.

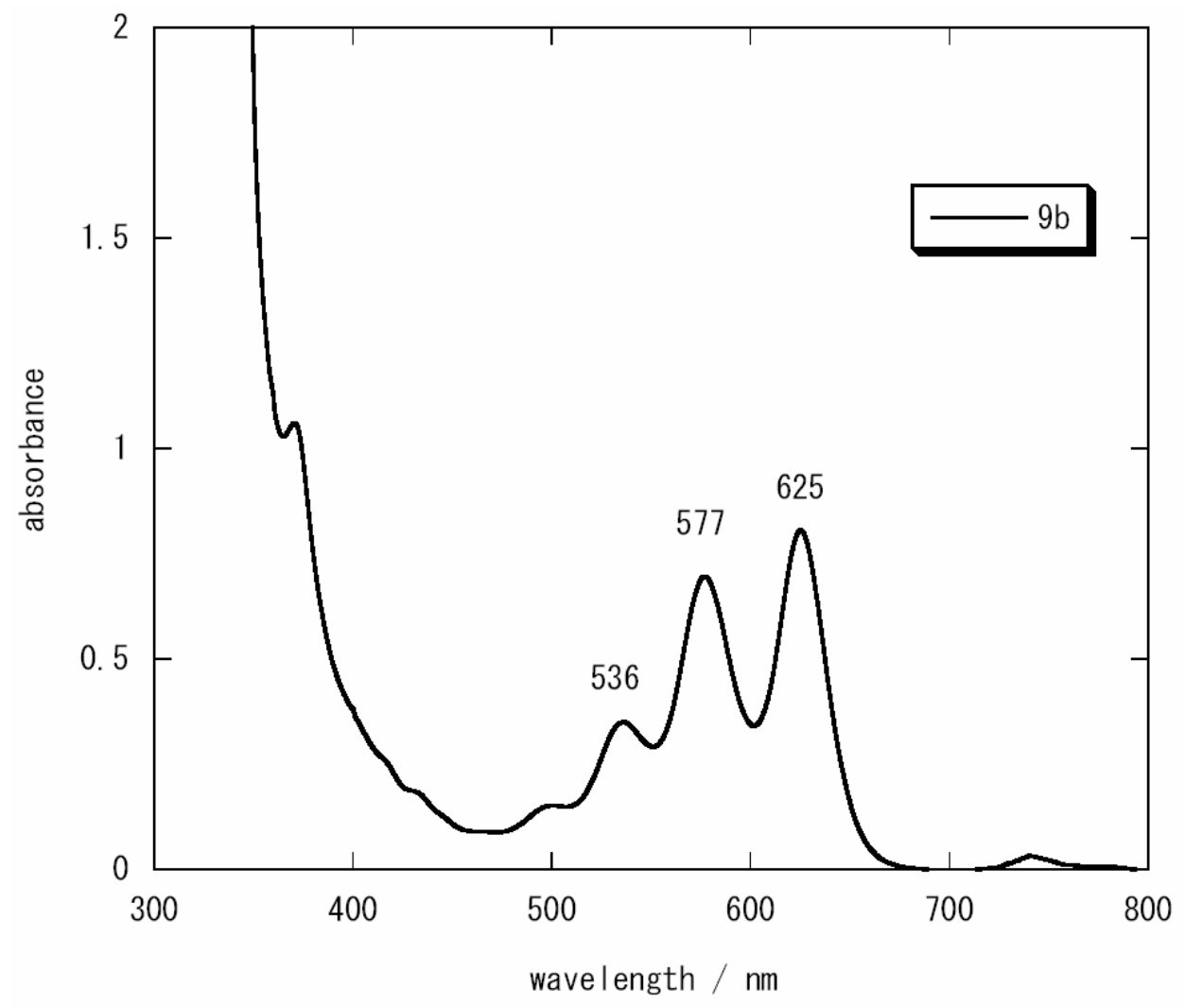

Figure 2. Absorption spectrum of pentacene $\mathbf{9 b}$ in THF at rt. 


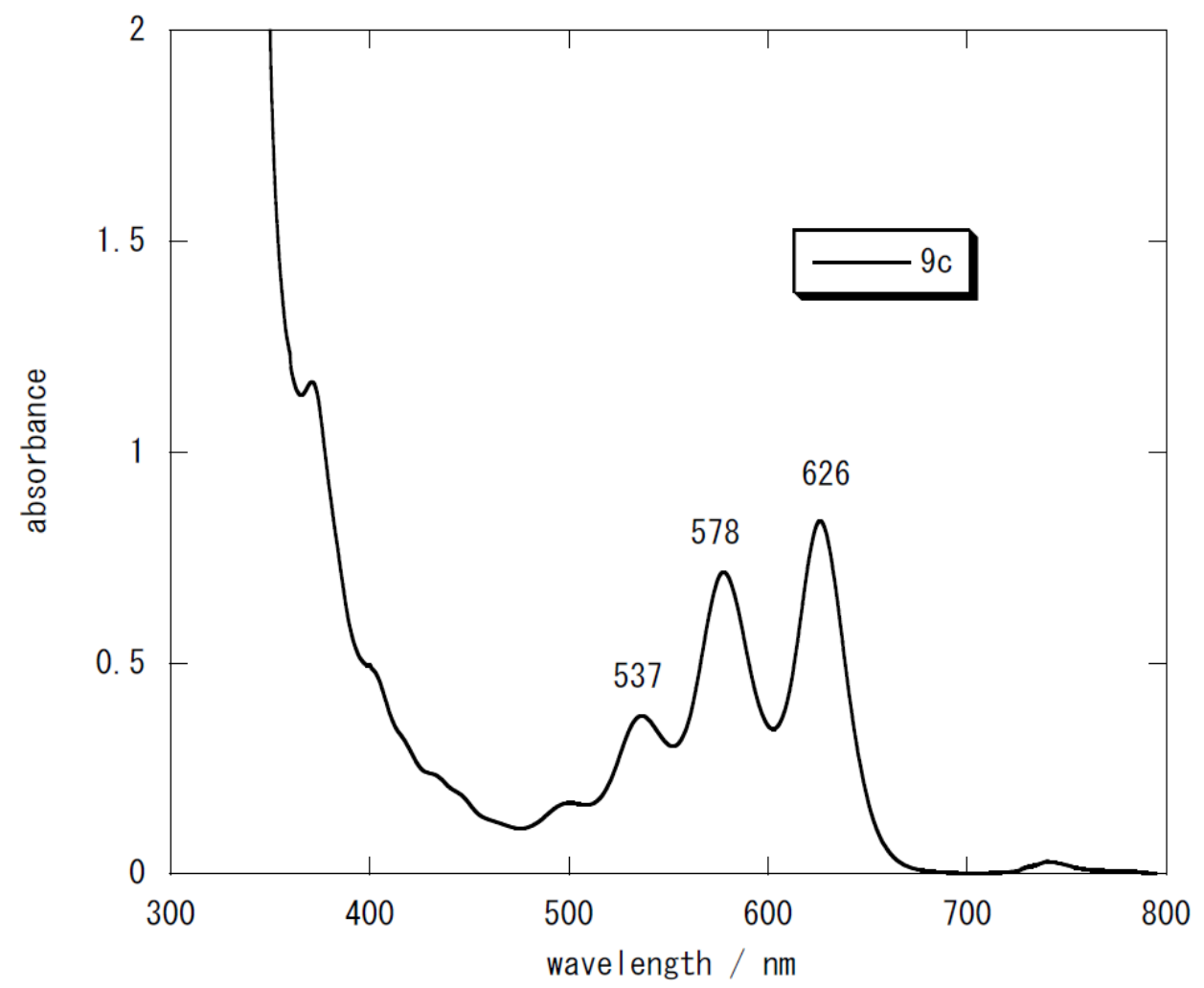

Figure 3. Absorption spectrum of pentacene $9 \mathrm{c}$ in THF at rt.

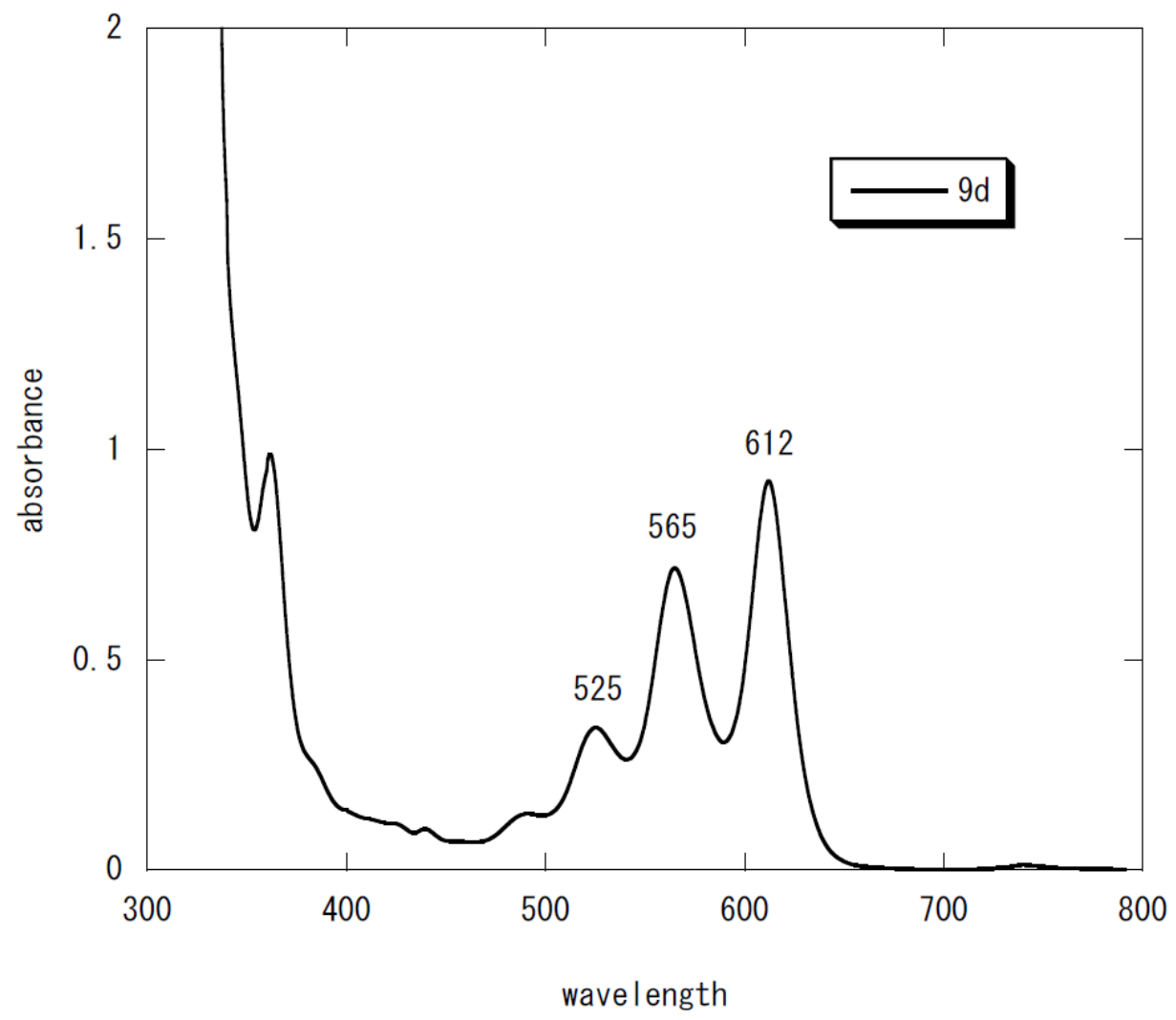

Figure 4. Absorption spectrum of pentacene 9d in THF at rt. 


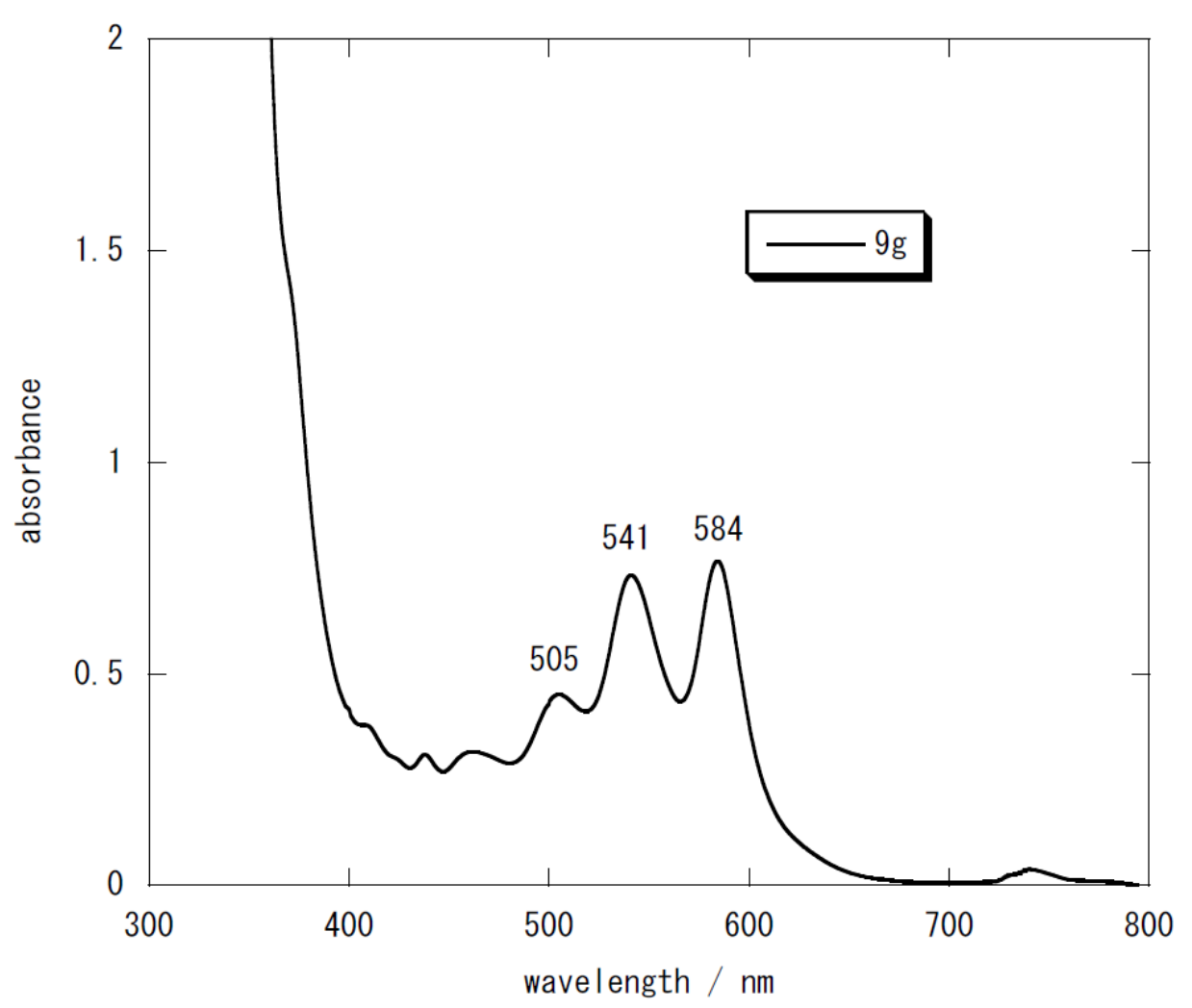

Figure 5. Absorption spectrum of pentacene $\mathbf{9 g}$ in THF at rt.

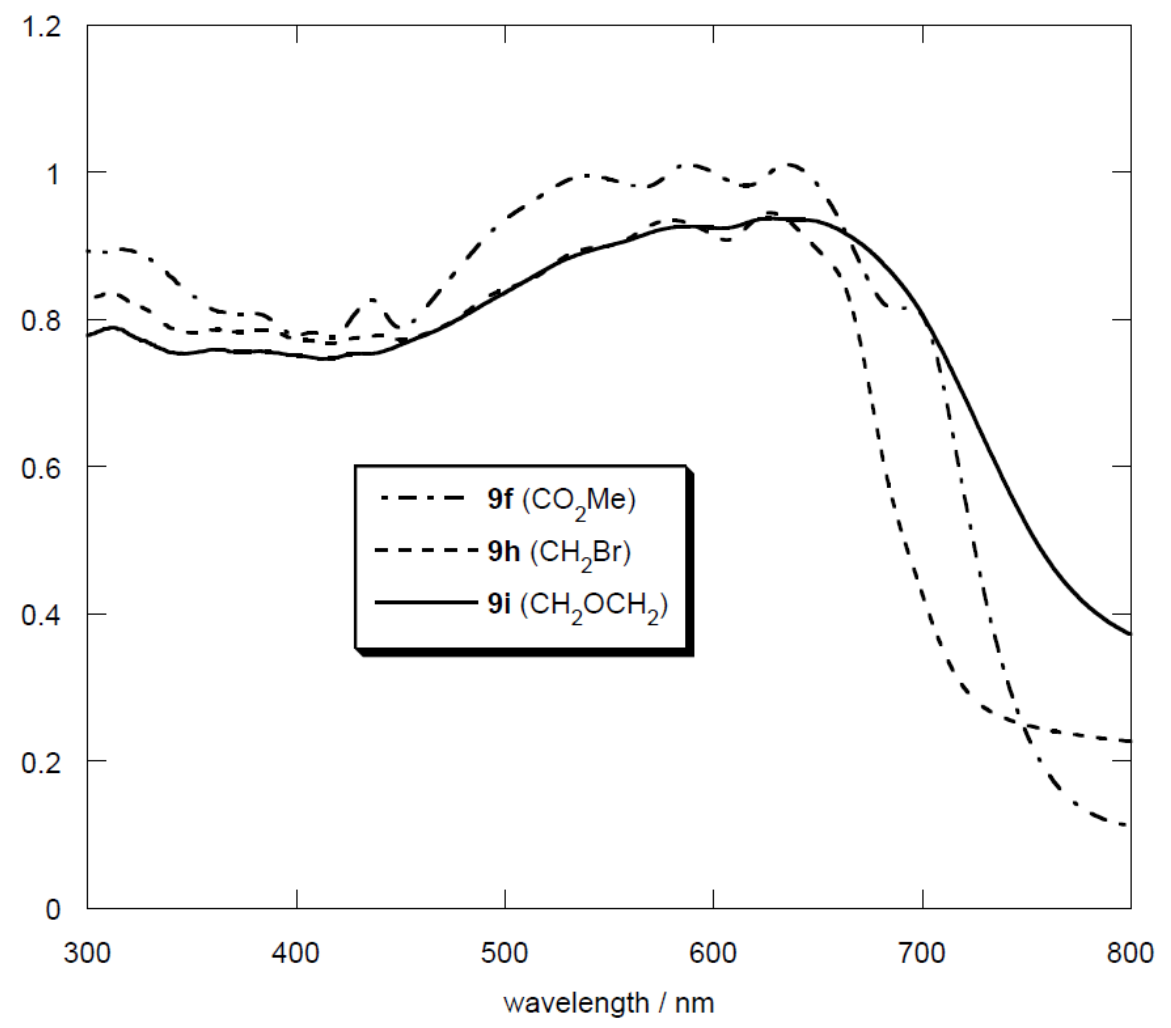

Figure 6. Absorption spectra of pentacenes $9 \mathbf{f}, 9 \mathbf{h}$, and, $9 \mathbf{i}$ in solid state at rt. 